\title{
SUBESPAÇOS INVARIANTES EM ALGUMAS ÁLGEBRAS BÁRICAS
}

\author{
Juaci Picanço da Silva
}

Tese apresentada

ao

Instituto de Matemática e Estatística

da

Universidade de São Paulo

para a obtenção do grau de Doutor

em

Matemática

Área de Concentração: Álgebra

Orientador: Prof. Dr. Roberto Celso Fabrício Costa

Durante a elaboraçào deste trabalho. o autor recebeu apoio financeiro da C'APES'

- São Paulo, dezembro de 1998 - 
Este exemplar corresponde à redação final da tese devidamente corrigida e defendida por Juaci Picanço da Silva e aprovada pela comissão julgadora.

São Paulo, 26 de janeiro de 1999.

Banca examinadora:

- Prof. Dr. Roberto Celso Fabrício Costa (Orientador) - IME - USP

- Prof. Dr. Alexandre Grishkov - IME - USP

- Prof. Dr. Antônio Paques - IMECC - UNICAMP

- Prof. Dr. Roseli Arbach Fernandes de Oliveira - FEIS - UNESP

- Prof. Dr. Ivan Shestakov - NOVOSIBIRSK UNIV. 
¿ Fofa e à Fefê 


\section{Agradecimentos}

Tenho muitas razòes pelas quais devo agradecer ao professor Roberto Costa. Por ele ter me aceitado como orientando quando tinha vários outros alunos. Pela dedicação com que nos orientava: houve um período que os nossos seminários eram diários. Mesmo depois de ter sofrido um grave problema de saúde e ter se submetido a uma desgastante e perigosa cirurgia, ele continuou cuidando de seus orientandos. Para mim, foi muito gratificante ter a oportunidade de conviver e ver como é o trabalho de um profissional como ele.

Gostaria de agradecer também às seguintes pessoas, sem a ajuda das quais, eu não conseguiria realizar este trabalho:

A minha esposa Ana Lúcia que se encarregou de muitas tarefas minhas e enfrentou sozinha muitos dos nossos problemas para que eu pudesse estudar e teve, juntamente com a nossa filha, a Fernanda, que tolerar intermináveis momentos de ausência de minha parte.

À Lúcia Ikemoto que me convenceu a estudar álgebras não associativas, apresentou-me o professor Roberto Costa, sugeriu várias mudanças neste trabalho e tirou muitas das minhas dúvidas. Além disso, em várias situações fora da universidade. pude contar com a sua ajuda e a do seu namorado, o Ricardo.

Ao Ivo Basso, com quem estudei o problema da invariância em álgebras que nào sào de Bernstein.

Ao Juan Carlos que. no pouco tempo que discutimos algumas propriedades dos Psubespaços, sugeriu algumas modificaçòes que melhoraram certos resultados.

Ao Jair e ao Orlando que tantas vezes me ajudaram na difícil convivência com os computadores. Quase tudo que sei sobre linguagem C e ${ }^{\mathrm{T}} \mathrm{T} \mathrm{EX}$ aprendi com eles.

Além dos já mencionados. uma série de outros amigos também contribuíram para tornar mais agradável esta fase da minha vida. Cito aqui alguns deles: C'ristina Vaz, Cristina Wolff, Claus, Fátima. Márcio. Adriana, Pablo, Roberto, Panzarelli. Lintz, Osvaldo, Marcia. Alan, Ana Cládia, Guzzo, Maria, Fábio S., Major, Ayumi, Fábio Beleza, Roseli, Carlos.

Gostaria também de agradecer a todas as pessoas que. com o seu trabalho. nobre on humilde, fazem o IME funcionar e ficar cada vez melhor. 


\section{Abstract}

In this work, we will introduce certain subspaces of the kernel of some baric algebras $(A, \omega)$ and among them, the Bernstein algebras. The set $\operatorname{Ip}(A)$ of idempotents of weight 1 of the algebras which we consider is not empty and each $e \in \operatorname{Ip}(A)$ determines a decomposition of $A$ which has the following form: $A=K e \pm U_{e} \oplus V_{e}$, where $K e, U_{e}$ and $V_{e}$ are the proper subspaces of the linear operator of $A$ defined by $L_{\epsilon}(x)=\epsilon x$. We will call P-subspaces those subspaces that have a polynomial expression in terms of $U_{\epsilon}$ and $V_{\epsilon}$, for instance: $L_{\epsilon} V_{\epsilon}, V_{e}^{2}$, $U_{e}^{2}+U_{e}^{3}, V_{\epsilon}^{3}+\left(U_{\epsilon} V_{\epsilon}\right) V_{\epsilon}+\left(U_{e} V_{e}\right)^{2}$. Our main purpose is to study the invariance of P-subspaces and also the invariance of dimension of P-subspaces under change of idempotent. We also consider a case $(A, \lambda)$, where $\lambda$ is only a linear form.

\section{Resumo}

Neste trabalho, introduzimos certos subespaços do núcleo de algumas álgebras báricas $(A, \omega)$, dentre elas as álgebras de Bernstein. $O$ conjunto $\operatorname{Ip}(A)$ dos idempotentes de peso 1 das álgebras que consideramos é nào vazio e cada $\epsilon \in \operatorname{Ip}(A)$ determina uma decomposiçào de $A$ da seguinte forma: $A=K^{\prime} \epsilon \oplus U_{\epsilon} \oplus V_{\epsilon}$. onde $K^{*} \epsilon, V_{\epsilon}$ e $V_{\epsilon}$ sào os subespaços próprios do operador linear de $A$ definido por $L_{e}(x)=\epsilon x$. C'hamamos de P-subespaços aos subespaços que possuem uma expressào polinomial em termos de $U_{e}$ e $V_{\epsilon}$, por exemplo: $L_{\epsilon} I_{e}, V_{\epsilon}^{2}$, $\tau_{e}^{2}+U_{e}^{3}, V_{\epsilon}^{3}+\left(U_{e} V_{\epsilon}\right) V_{\epsilon}+\left(U_{e} V_{e}\right)^{2}$. Nosso principal objetivo é estudar a invariância dos Psubespaços e também a invariância da dimensào dos P-subespaços com relaçào à mudança do idempotente. Também consideramos um caso $(., \lambda)$. onde $\lambda$ é apenas uma forma linear. 


\section{Sumário}

$\begin{array}{ll}\text { Introdução } & 3\end{array}$

0 Preliminares $\quad \mathbf{5}$

0.1 Álgebras de Bernstein . . . . . . . . . . . . . . . . . . . . . . . . . . . . . . .

0.2 Álgebras de Bernstein-Jordan . . . . . . . . . . . . . . . . 11

1 P-subespaços $\quad 13$

1.1 P-subespaços de álgebras de Bernstein . . . . . . . . . . . . . . . . . 14

1.2 Nilpotência e P-subespaços . . . . . . . . . . . . . . . . 18

2 Invariância de P-subespaços em álgebras de Bernstein 19

2.1 Exemplos de P-subespaços invariantes . . . . . . . . . . . . . . . 19

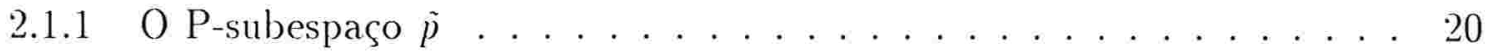

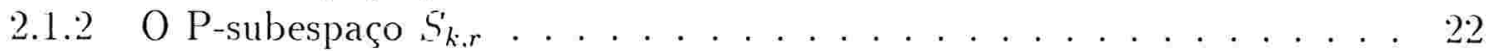

2.2 Exemplos de P-subespaços não invariantes . . . . . . . . . . . . . . 24

2.3 Invariância em álgebras de Bernstein satisfazendo $U^{2} V=0$ e $(u v) v=0 \ldots 28$

2.4 Invariância em álgebras de Bernstein-Jordan . . . . . . . . . . . . . . 36

2.5 Invariância em álgebras de Bernstein . . . . . . . . . . . . . . 38

2.5.1 P-subespaços de grau $\leq 3 \ldots \ldots \ldots \ldots \ldots$

3 Invariância de P-subespaços em outras álgebras báricas $\quad 50$

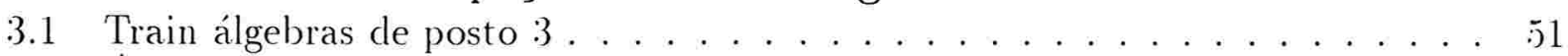

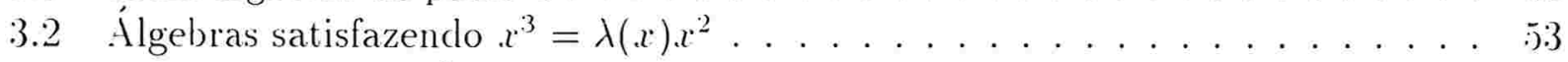

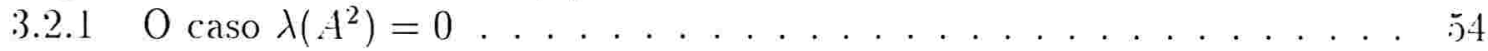

$3.2 .2 \quad 0$ caso $\lambda\left(A^{2}\right) \neq 0 \ldots \ldots \ldots \ldots \ldots$

3.3 Algebras satisfazendo $\left(x^{2}\right)^{2}=\omega(x) x^{3} \ldots \ldots \ldots \ldots . \ldots 2$

3.4 Álgebras satisfazendo $\left(x^{2}\right)^{2}=w\left(x^{3}\right) x \ldots \ldots \ldots \ldots$

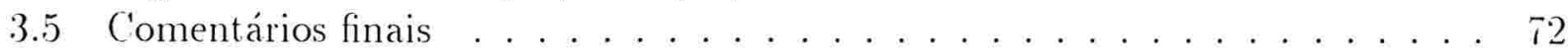


Referências Bibliográficas

$\begin{array}{ll}\text { Índice de Símbolos } & 76\end{array}$

$\begin{array}{ll}\text { Índice Remissivo } & 77\end{array}$ 


\section{Introdução}

O principal objetivo deste trabalho é encontrar invariantes para uma álgebra de Bernstein $(A, \omega)$. Faremos isto utilizando certos subespaços de $A$, chamados P-subespaços, que sào construídos a partir de uma decomposição de Peirce de $A$. Se $A=I \epsilon \in U \oplus V$ é uma tal decomposição relativa ao idempotente $\epsilon$, entào os seguintes subespaços: $\left[+V, U V, U^{2}, V^{3}\right.$, $\left[V \oplus V,\left((U V) V+U^{2} V\right) \oplus U^{2}\right.$. etc. são exemplos de P-subespaços. Ou seja, os P-subespaços são, em poucas palavras, subespaços de $A$ que possuem uma expressão polinomial em termos de $U$ e $V$. Assim, cada P-subespaço está associado ao idempotente escolhido para se fazer a decomposição de Peirce. Entretanto, mostraremos que alguns deles não dependem desta escolha. Tais subespaços são invariantes de A. Ainda, as dimensòes de certos P-subespaços nào dependem da mudança de idempotente e, portanto são invariantes numéricos de $A$.

Inicialmente estudamos os P-subespaços na subclasse das álgebras de Bernstein que satisfazem $U^{2} V=0$ e $(u v) v=0$ para quaisquer $u \in U$ e $v \in V$. Mostraremos que todos os P-subespaços das álgebras de Bernstein desta subclasse têm dimensão invariante e encontraremos uma condição de simples verificaçào. que é necessária e suficiente, para que um dado $\mathrm{P}$-subespaço seja, ele próprio. invariante. Tais resultados provavelmente são válidos para outras subclasses, mas mostramos que, num certo sentido, não é possível aumentar esta subclasse e ainda ter os mesmos resultados. Além disso, a subclasse que estudamos é bastante grande. pois contém as álgebras de Bernstein-Jordan. Este fato foi de grande utilidades para o estudo dos P-subespaços na classe inteira, visto que em toda álgebra de Bernstein existe um ideal $L$ tal que o quociente por $L$ é uma álgebra de Bernstein-.Jordan. Com isso é possível provar alguns resultados para uma álgebra de Bernstein qualquer usando o que sabemos para as álgebras de Bernstein-.Jordan. Por exemplo, desta forma prova-se que todo P-subespaço de uma álgebra de Bernstein, contido em $V$, tem dimensão invariante.

Para as álgebras de Bernstein-Jordan foram obtidos os melhores resultados e todos eles săo válidos para as train álgebras de posto 3 .

Depois observamos que o método utilizado para encontrar invariantes nas álgebras de Bernstein e train de posto 3. podia ser aplicado para obter invariantes em outras classes de álgebras báricas que possuem uma decomposiçào de Peirce. Também estudamos álgebras 
munidas de uma forma linear apenas.

Este trabalho é a continuaçào do estudo de subespaços invariantes, iniciado por duas alunas, a Roseli Oliveira e a Maria Luiza Lelis. que estudaram esta questão em train álgebras de posto 3 e Bernstein, respectivamente. 


\section{Capítulo 0}

\section{Preliminares}

Neste capítulo. citaremos os conceitos e resultados básicos sobre álgebras de Bernstein e provaremos alguns fatos que serão utilizados, principalmente, nos Capítulos 1 e 2.

Seja A uma álgebra nào necessariamente associativa sobre um corpo $K$. Neste trabalho consideraremos sempre $\operatorname{car}\left(K^{\circ}\right) \neq 2$ e $A$ de dimensão finita. As potências principais de um elemento $a \in A$ sào definidas recursivamente por $a^{1}=a$ e $a^{n+1}=a a^{n}$. para todo inteiro positivo $n$. Em geral, temos $a^{m} a^{n} \neq a^{m+n}$.

Se $S$ é um subconjunto de $A$, usaremos a notação $\left\langle S^{\prime}\right\rangle$ para indicar o subespaço de $A$ gerado por $S$. Dados dois subespaços $X$ e $Y$, definimos o subespaço $X Y$ por

$$
X Y=\langle x y: x \in X \text { e } y \in Y\rangle
$$

Muitas vezes utilizaremos as seguintes observaçòes:

Observação 0.1 Se X' $\mathcal{X}$ um conjunto de geradores do subespaço X $\in \mathcal{Y}$ é um conjunto de geradores do subespaço $Y$ então $X^{\prime} Y=\left\langle x y ; x \in X^{\prime} \in y \in \mathcal{Y}\right\rangle$.

Observação 0.2 Se $X . Y$ $Y$ sĩo subespaşos de uma álgebra A entäo $X(Y+Z)=X Y+$ $\mathrm{X} Z$.

A segunda observaçào é conseqüência das igualdades $x(y+z)=x y+x z$ e $x_{1} y+x_{2} z=$ $x_{1}(y+0)+x_{2}(0+z)$, onde $x \cdot x_{1}, x_{2} \in X . y \in Y$ e $z \in Z$.

Para subespaços usamos também a notação de potências principais: $X^{1}=X$ e $X^{n}=$ $Y\left(X^{n-1}\right)$.

Em várias ocasiōes usaremos o seguinte lema:

Lema 0.1 Seja X um subespago de uma rilgebra A. Se A é comutatica entrio 
(a) $\mathrm{X}^{2}=\left\langle x^{2}: x \in \mathrm{X}\right\rangle$ :

(b) $\mathrm{X}^{r}+\mathrm{X}^{2}=\left\langle x+x^{2}: x \in \mathrm{X}\right\rangle$.

Demonstração: Sejam $x . x_{1}, x_{2} \in X$. A parte $(a)$ segue da fórmula $x_{1} x_{2}=\frac{1}{2}\left(\left(x_{1}+x_{2}\right)^{2}-\right.$ $\left.x_{1}^{2}-x_{2}^{2}\right)$ e a parte $(b)$ é conseqüência de $(a)$ e das identidades $x^{2}=\frac{1}{2}\left(x+x^{2}\right)+\frac{1}{2}\left(-x+(-x)^{2}\right)$ e $x=\left(x+x^{2}\right)-x^{2}$.

Quando existe um homomorfismo não nulo $\omega: A \rightarrow K$, o par $(A, \omega)$ é chamado de álgebra bárica, o homomorfismo w é dito função peso de $A$ e o escalar $\omega(a)$, com $a \in A$, é o peso do elemento $a$. Chamamos de núcleo de $A$ ao kernel de $\omega$ e usamos a notaçào $N=\operatorname{ker} \omega$.

Se $I$ é um ideal de $A \operatorname{com} I \subseteq N$ entào $\bar{\omega}: \bar{A} \rightarrow K$. definido por $\bar{\omega}(x+I)=\omega(x)$, para todo $x \in A$. é um homomorfismo da álgebra quociente $\bar{A}=1 / I$ em $K$. Portanto, $(\bar{A} . \bar{\omega})$ é uma também álgebra bárica.

\section{1 Álgebras de Bernstein}

Uma álgebra bárica $(A, \omega)$ sobre um corpo $K$ é chamada de álgebra de Bernstein se $A$ é comutativa e $\left(x^{2}\right)^{2}=\omega\left(x^{2}\right) x^{2}$, para todo $x \in A$. Portanto, os elementos do núcleo $N$ de $A$ satisfazem a identidade

$$
\left(x^{2}\right)^{2}=0
$$

e as seguintes linearizaçòes:

$$
\begin{aligned}
x_{1}^{2}\left(x_{1}, x_{2}\right) & =0 \\
x_{1}^{2}\left(x_{2}, x_{3}\right)+2\left(x_{1}, x_{2}\right)\left(x_{1}, x_{3}\right) & =0 \\
\left(x_{1}, x_{2}\right)\left(x_{3} x_{4}\right)+\left(x_{1} x_{3}\right)\left(x_{2} x_{4}\right)+\left(x_{1}, x_{4}\right)\left(x_{2}, x_{3}\right) & =0
\end{aligned}
$$

$\operatorname{com} x_{1}, x_{2}, x_{3}, x_{4} \in \mathrm{X}^{\circ}$.

Em toda álgebra de Bernstein, $\omega$ é o único homomorfismo não nulo de $A$ em $K$. Por isso. muitas vezes. nos referimos a uma álgebra de Bernstein escrevendo apenas $A$ ao invés de $(A, \omega)$. O núcleo $N$ é um ideal de $A$ cle codimensào 1 e existe pelo menos um idempotente de peso 1 de $A$. Cada idempotente $e$ de peso 1 determina uma decomposição da álgebra:

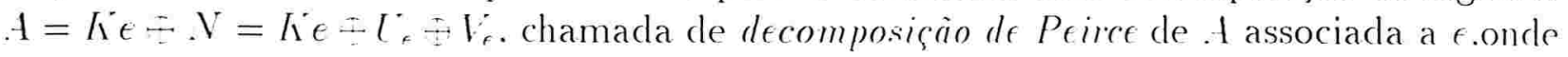

$$
\begin{aligned}
U_{\epsilon} & =\left\{u ; \epsilon u=\frac{1}{2} u\right\} \\
V_{\epsilon} & =\{u ; \epsilon u=0\}
\end{aligned}
$$


são subespaços de $A$ e satisfazem as inclusòes

$$
U_{e} V_{e} \subseteq U_{e} \quad V_{\epsilon}^{2} \subseteq U_{e} \quad U_{e}^{2} \subseteq V_{e}
$$

e a igualdade

$$
U_{\epsilon} V_{\epsilon}^{-2}=0
$$

Os elementos $u$ de $U_{e}$ e $v$ de $V_{e}$ satisfazem as seguintes identidades:

$$
\begin{aligned}
u^{3} & =0 \\
u c^{2} & =0 \\
u(u v) & =0 \\
(u)^{2} & =0 \\
u^{2} v^{2} & =0
\end{aligned}
$$

Linearizando estas identidades obtemos

$$
\begin{aligned}
u_{1}^{2} u_{2}+2 u_{1}\left(u_{1} u_{2}\right) & =0 \\
u_{1}\left(u_{2} u_{3}\right)+u_{2}\left(u_{3} u_{1}\right)+u_{3}\left(u_{1} u_{2}\right) & =0 \\
u\left(v_{1} v_{2}\right) & =0 \\
u_{1}\left(u_{2} v^{\prime}\right)+u_{2}\left(u_{1} v\right) & =0 \\
\left(u_{1} v\right)\left(u_{2} v^{\prime}\right)=\left(u v_{1}\right)\left(u v_{2}\right) & =0 \\
\left(u_{1} u_{2}\right)\left(v_{1} v_{2}\right) & =0
\end{aligned}
$$

onde $u . u_{i} \in U_{\epsilon}$ e $v, v_{j} \in V_{e}(i=1,2,3 ; j=1,2)$.

Usando as identidades (12), (13) e (15). prova-se o seguinte resultado para subespaços do núcleo de uma álgebra de Bernstein.

Proposição 0.1 Sejam $X_{1}, X_{2}, X_{3} \subseteq U_{\epsilon} \in W \subseteq V_{\epsilon}$ subespaços de uma álgebra de Bernstein $A=K_{\epsilon} \oplus U_{\epsilon} \oplus V_{\epsilon}$. Então
(a) $X_{1}^{2} X_{2} \subseteq X_{1}^{\prime}\left(X_{1}, X_{2}^{\prime}\right)$;
(b) $X_{1}\left(X_{2} \cdot X_{3}\right) \subseteq X_{2}\left(X_{3} X_{1}\right)+X_{3}\left(X_{1} X_{2}\right)$ :
(c) $X_{1}\left(X_{2} W\right)=X_{2}\left(X_{1} W\right)$. 
Demonstração: Segue da parte $(a)$ do Lema 0.1 e da Observação 0.1 que $X_{1}^{2} X_{2}$ é gerado por elementos da forma $x_{1}^{2} x_{2} \operatorname{com} x_{1} \in X_{1}$ e $x_{2} \in X_{2}$. Agora. pela identidade (12), $x_{1}^{2} x_{2} \in$ $X_{1}\left(X_{1} X_{2}\right)$. Assim, $X_{1}^{2} X_{2} \subseteq X_{1}\left(X_{1} X_{2}\right)$. As demonstrações de $(b)$ e $(c)$ são análogas.

O conjunto de todos os idempotentes de $A$ de peso 1 é dado por

$$
\operatorname{Ip}(A)=\left\{e+u+u^{2} ; u \in U_{e}\right\}
$$

Se $f_{u}=e+u+u^{2}$ é outro idempotente de $A, \operatorname{com} u \in U_{e}$, então

$$
\begin{aligned}
U_{f_{u}} & =\left\{x+2 u x ; x \in U_{e}\right\} \\
V_{f_{u}} & =\left\{v-2 u v-2 u^{2} v ; v \in V_{e}\right\}
\end{aligned}
$$

e as aplicaçòes lineares $\sigma_{u}: U_{e} \rightarrow U_{f_{u}}$ e $\tau_{u}: V_{\epsilon} \rightarrow V_{f_{u}}$ definidas por

$$
\begin{aligned}
\sigma_{u}(x) & =x+2 u x \\
\tau_{u}(v) & =v-2 u v-2 u^{2} v
\end{aligned}
$$

são isomorfismos de espaços vetoriais. Portanto, a dimensão dos subespaços $U_{e}$ e $V_{e}$ não depende da escolha do idempotente. Assim, $\operatorname{dim} U_{\epsilon}$ e $\operatorname{dim} V_{\epsilon}$ são invariantes numéricos de A. Dizemos que o par $\left(1+\operatorname{dim} U_{e}, \operatorname{dim} V_{e}\right)$ é o tipo de $A$. Na maioria dos casos, quando não houver possibilidade de confusão, omitimos o índice $\epsilon \operatorname{em} U_{e}, V_{\epsilon}$ e o índice $u$ em $f_{u}, \sigma_{u}$ e $\tau_{u}$.

Se $(A, \omega)$ é uma álgebra de Bernstein com decomposição de Peirce $A=K e \oplus U \oplus V$. entào $\left(A^{2},\left.\omega\right|_{A^{2}}\right)$ é uma álgebra de Bernstein com decomposição de Peirce associada a $\epsilon$ dada por $A^{2}=K \in \oplus U \notin U^{2}$. Dizemos que $A$ é nuclear se $A=A^{2}$ ou equivalentemente se $V=U^{2}$.

Quando $U^{2}=0$, dizemos que $A$ é excepcional. No caso em que $U V=V^{2}=0$, $A$ é dita normal.

Todos os resultados citados anteriormente sobre álgebras de Bernstein podem ser encontrados em [9] e [19].

Dada uma família $\left\{X_{\alpha}\right\}_{\alpha \in \mathcal{I}}$ de subespaços de uma álgebra. fazendo a soma e a intersecçào de todos os seus subespaços, obtemos dois novos subespaços associados à família dada. A próxima proposição nos diz o que é obtido dessa maneira para $\left\{U_{f}\right\}_{f \in \operatorname{Ip}(A)}$ e $\left\{V_{f}\right\}_{f \in \operatorname{Ip}(A)}$. Na verdade, a proposição seguinte é conseqüência de um fato mais geral que demonstraremos no Capítulo 2, mas ela é apresentada agora por conveniência.

Proposição 0.2 Seja $1=K e \pm U \geq V$ uma álgebra de Bemstcin. Então

(a) $\bigcap_{f \in \operatorname{Ip}(A)} L_{f}^{*}=U \cap \operatorname{ann} U^{\prime}$ : 
(b) $\bigcap_{f \in \operatorname{Ip}(A)} V_{f}=V \cap \operatorname{ann}\left(U \oplus U^{2}\right)$;

(c) $\sum_{f \in \operatorname{Ip}(A)} U_{f}=U \oplus U^{2}$

(d) $\sum_{f \in \operatorname{Ip}(A)} V_{f}=\left(U V+U^{2} V\right) \oplus V$

Demonstração: A fórmula (a) faz parte da Proposição 3.4 de [3]. Mostremos (b). Seja $\epsilon \in \operatorname{Ip}(A)$ fixo. Se $w \in \bigcap_{f \in \operatorname{Ip}(A)} V_{f}$ entào, para cada $u \in U_{e}$, existe $v \in V_{e}$ tal que $w=$ $v-2\left(u+u^{2}\right) v$. De onde segue que $w=v$ e $\left(u+u^{2}\right) v=0$. Agora, usando a parte $(b)$ do Lema 0.1. concluímos que $w \in V_{e} \cap \operatorname{ann}\left(U_{\epsilon} \div U_{e}^{2}\right)$. Assim,

$$
\bigcap_{f \in \operatorname{Ip}(A)} V_{f} \subseteq V_{\epsilon} \cap \operatorname{ann}\left(U_{\epsilon} \Phi U_{\epsilon}^{2}\right)
$$

Por outro lado, se $v \in V_{e} \cap \operatorname{ann}\left(U_{e} \oplus U_{e}^{2}\right)$ entào. para todo $u \in U_{e}$, temos que $v=v-2(u+$ $\left.u^{2}\right) v \in V_{f_{u}}$. Logo, $v \in \bigcap_{f \in \operatorname{Ip}(A)} V_{f}$. Assim,

$$
V_{e} \cap \operatorname{ann}\left(U_{\epsilon} \oplus L_{\epsilon}^{-2}\right) \subseteq \bigcap_{f \in \operatorname{Ip}(A)} V_{f}
$$

o que conclui a demonstraçào da parte $(b)$. Mostremos agora $(c)$. Como $U_{f_{u}}=\{x+2 u x ; x \in$ $\left.U_{\epsilon}\right\}$. para todo $u \in U_{e}$, temos que

$$
\sum_{f \in \operatorname{Ip}(A)} U_{f}=\sum_{u \in U_{e}^{\prime}}\left\langle\dot{x}+2 u x ; x \in U_{\epsilon}\right\rangle
$$

Segue dai que $\sum_{f \in \operatorname{Ip}(A)} U_{f} \subseteq U_{e} \oplus U_{\epsilon}^{2}$. Por outro lado, é evidente que $U_{\epsilon} \subseteq \sum_{f \in \operatorname{Ip}(A)} U_{f}$ e, segue de

$$
u^{2}=\frac{1}{2}\left(u+2 u^{2}\right)-\frac{1}{2} u=\frac{1}{2} \sigma_{u}(u)-\frac{1}{2} u \in U_{f_{u}}+U_{\epsilon} \subseteq \sum_{J \in \operatorname{Ip}(A)} U_{f}
$$

que $I_{\epsilon}^{2} \subseteq \sum_{f \in \operatorname{lp}(-1)} U_{f}$. Para mostrar $(d)$, observemos que

$$
\sum_{f \in I_{\mathrm{P}}(-A)} V_{f}=\sum_{u \in l_{e}^{*}}\left\langle v-2 u v-2 u^{2} v ; v \in V_{\epsilon}\right\rangle
$$


Portanto, $\sum_{f \in \operatorname{Ip}(A)} V_{f} \subseteq\left(U_{\epsilon} V_{\epsilon}+\left(U_{\epsilon}^{2} V_{e}\right) \oplus V_{e}\right.$. Por outro lado. temos que $V_{\epsilon} \subseteq \sum_{f \in \operatorname{Ip}(A)} V_{f}$. Agora segue das Observações 0.1 e 0.2 e do Lema 0.1 que

$$
U_{e} V_{e}+U_{e}^{2} V_{e}=\left(U_{\epsilon}+C_{\epsilon}^{-2}\right) V_{e}=\left\langle\left(u+u^{2}\right) v ; u \in U_{\epsilon} \mathrm{e} v \in V_{\epsilon}\right\rangle \subseteq \sum_{f \in \operatorname{Ip}(A)} V_{f}
$$

pois $\left(u+u^{2}\right) v=\frac{1}{2} v-\frac{1}{2}\left(v-2\left(u+u^{2}\right) v\right)=\frac{1}{2} v-\frac{1}{2} \tau_{u}(v) \in \dot{V}_{e}+V_{f_{u}}$.

Definimos os subespaços $\tilde{l^{*}}$ e $\tilde{V}$ como segue

$$
\begin{aligned}
& \tilde{U}=\sum_{f \in \operatorname{Ip}(A)} U_{f} \\
& \tilde{V}=\sum_{f \in \operatorname{Ip}(A)} V_{f}
\end{aligned}
$$

A proposição anterior nos dá uma descrição mais simples de $\tilde{l^{*}}$ e $\tilde{V}$. isto é,

$$
\begin{aligned}
\tilde{U} & =U \oplus U^{2} \\
\tilde{V} & =\left(U V+U^{2} V\right) \oplus V
\end{aligned}
$$

É claro da definiçào que $\tilde{U}$ e $\tilde{V}$ não dependem da escolha de um particular idempotente. Portanto, $U \oplus U^{2}$ e $\left(U V+U^{2} T^{\circ}\right) \boxplus$ não dependem do idempotente. Este fato já é conhecido. uma vez que $U \oplus U^{2}$ é o núcleo de $A^{2}$ e, foi provado em [8] que. para o train polinômio $p=x^{3}-\frac{1}{2} \omega(x) x^{2}-\frac{1}{2} \omega(x)^{2} x \cdot \operatorname{temos}\left(U V+C^{2} V\right) \oplus V=\{a \in A: f(a)=0$ para qualquer $f \in$ $\left.J_{p}\right\}$, onde $J_{p}=\left\{f \in \mathcal{A}^{*}: f(p(a))=0\right\}$.

Dois outros subespaços que não dependem do idempotente escolhido sào: $N=U \oplus V \mathrm{e}$ $P=\left(U V+V^{2}\right) \oplus V$. Sabemos que $N=$ ker $\omega$ e é bem conhecido que $P$. chamado de ideal de Etherington, é o ideal gerado por $\left\{x^{2}-\omega(x) x: x \in A\right\}$.

O subespaço $L=U^{\prime} \cap$ ann $U^{\prime}$ é um ideal de $A$ e. da parte $(a)$ da proposição anterior. temos que

$$
L=\bigcap_{f \in \operatorname{Ip}(A)} U_{f}
$$

Assim, $L$ é invariante quanto à escolha do idempotente. A importância deste ideal está no fato de $1 / L$ ser uma álgebra de Jordan. como veremos na próxima seçào. 


\section{2 Álgebras de Bernstein-Jordan}

As álgebras de Bernstein-Jordan são álgebras de Bernstein $(A, \omega)$, onde $A$ é uma álgebra de Jordan, isto é, satisfaz a identidade $(x y) x^{2}=x\left(y x^{2}\right)$. O resultado seguinte é a Proposição 3.1 de $[3]$.

Proposição 0.3 Seja $(A, \omega)$ uma álgebra de Bernstein. São equivalentes:

(a) A é uma álgebra de Jordan;

(b) $I_{\epsilon}^{2}=0$. para todo $e \in I p(A)$;

(c) Para todo $e \in I p(A)$, tem-se $V_{\epsilon}^{2}=0 \in(u v) v=0$, para quaisquer $u \in U_{e}$ ev $\in V_{\epsilon}$ :

(d) Existe $\epsilon \in I p(A)$ tal que $V_{e}^{2}=0 \in(u v) v=0$. para todo $u \in U_{e} \in v \in V_{e}$;

(e) A satisfa z a identidade $x^{3}=\omega(x) x^{2}$.

Segue desta proposição que as álgebras de Bernstein-Jordan, satisfazem a equação

$$
(u v) v=0
$$

e, conseqüentemente, a linearizaçào desta

$$
\left(u v_{1}\right) v_{2}+\left(u v_{2}\right) v_{1}=0
$$

onde $u \in U$ e $v, v_{1}, v_{2} \in V$. Além disso, segue da parte $(\epsilon)$ que

$$
\begin{aligned}
x^{3} & =0 \\
x_{1}^{2} x_{2}+2 x_{1}\left(x_{1} x_{2}\right) & =0 \\
x_{1}\left(x_{2} x_{3}\right)+x_{2}\left(x_{3} x_{1}\right)+x_{3}\left(x_{1} x_{2}\right) & =0
\end{aligned}
$$

para quaisquer elementos $x, x_{1}, x_{2}, x_{3}$ do núcleo de uma álgebra de Bernstein-.Jordan.

A próxima proposição é conseqüência da identiclade (28).

Proposição 0.4 Sejam $X \subseteq U \in W_{1}, W_{2} \subseteq V$ subespaços de uma álgebra de BernsteinJordan $A=I \dot{A} \epsilon \amalg \oplus V$. Então $\left(X W_{1}\right) W_{2}=\left(X W_{2}\right) W_{1}$.

Seja I um ideal de uma álgebra de Bernstein (.f.w) contido em . V. Usando a parte (b) da Proposiçào 0.3 , temos que uma condiçào necessária e suficiente para que o quociente $.1 / I$ seja uma álgebra de Jordan é que $V_{e}^{2} \subseteq I$ para todo $\epsilon \in \operatorname{Ip}(. A)$. Sobre isto, temos o seguinte corolário (ver [5] e [6]). 
Corolário 0.1 Para toda álgebra de Bernstein $(A, \omega)$, a álgebra quociente $(A / L, \bar{\omega})$ é uma álgebra de Bernstein-Jordan.

Demonstração: Temos que $L \subseteq N$ e, de (14), segue que $V^{2} \subseteq L$. para toda decomposição de Peirce $A=L e \oplus U \oplus V$ de $A$. 


\section{Capítulo 1}

\section{P-subespaços}

Dados $X$ e $Y$ subespaços de uma $K$-álgebra comutativa $A$, os subespaços monomiais de $A$ determinados por $\mathrm{X}$ e $Y$ sào definidos recursivamente da seguinte maneira:

(1) $\mathrm{X}$ e $Y$ são eles próprios subespaços monomiais determinados por $\mathrm{X}$ e $Y$;

(2) Se $m_{1}$ e $m_{2}$ sào subespaços monomiais determinados por $X$ e $Y$, entào $m=m_{1} m_{2}$ é um subespaço monomial determinado por $X$ e $Y$. $Y$ :

Os seguintes subespaços são exemplos de subespaços monomiais determinados por $\mathrm{X}$ e

$$
\begin{aligned}
\mathrm{X}^{2} Y & =\left\langle\left(x_{1} x_{2}\right) y ; x_{1}, x_{2} \in X, y \in Y\right\rangle \\
\left(X^{\prime} Y\right) Y & =\left\langle\left(x y_{1}\right) y_{2}: x \in X^{\prime}, y_{1}, y_{2} \in Y\right\rangle \\
X^{3} & =\left\langle\left(x_{1} x_{2}\right) x_{3} ; x_{1}, x_{2}, x_{3} \in X\right\rangle \\
\left(X^{\prime} Y\right)^{2} & =\left\langle\left(x_{1} y_{1}\right)\left(x_{2} y_{2}\right): x_{1}, x_{2} \in X, y_{1}, y_{2} \in Y\right\rangle
\end{aligned}
$$

Chamamos de subespaço polinomial determinado por $\mathrm{X}$ e $\mathrm{Y}$ aos subespaços de $A$ que sào somas finitas de subespaços monomiais determinados por $\mathrm{X}$ e $Y$.

Seja $K\left[U . U^{\ddots}\right]$ a álgebra dos polinômios nas variáveis comutativas e não associativas $l^{*} \mathrm{e}$ $\lceil$ e seja $\mathcal{F}$ o subconjunto de $K[U, V]$ formado pelos polinômios sem termo constante e com todos os coeficientes iguais a 1 . A cada elemento $p(U, V)$ de $\mathcal{P}$ corresponde um subespaço polinomial determinado por $X$ e $Y$. De fato. basta substituir $U$ por $X$ e $V$ por $Y$. O subespaço polinomial assim obtido é denotado por $p(X . Y)$ ou simplesmente p. Quando Xe $Y$ estào fixados e nào houver possibilidade de confusào chamamos $p\left(X^{\prime} Y\right)$ de $P$-subespaço. Se $p(U, V)$ é um monômio. diremos que $p(X, Y)$ é um $P$-monômio. 
Para um elemento $p$ de $K[U, V]$ utilizamos a notaçào $\partial p$ para indicar o grau de $p$. Se $p=\sum_{i=1}^{k} m_{i}$, onde $m_{i}$ são monômios, definimos a ordem de $p$ como sendo $o(p)=\min \left\{\partial m_{i} ; i=\right.$ $1 \ldots, k\}$.

Sejam $I$ um ideal de $A$ e $\bar{X}=(X+I) / I$ e $\bar{Y}=(Y+I) / I$ subespaços da álgebra quociente $\bar{A}=A / I$. Os P-subespaços $p(X, Y)$ de $A$ estão relacionados com os P-subespaços $p(\bar{X}, \bar{Y})$ de $\bar{A}$ da seguinte maneira:

$$
p(\bar{X} \cdot \bar{Y})=(p(X, Y)+I) / I
$$

\subsection{P-subespaços de álgebras de Bernstein}

Nesta seçào, começamos o estudo dos P-subespaços $p\left(U_{\epsilon}, V_{\epsilon}\right)$ de álgebras de Bernstein (.A. $\omega)$, onde $U_{e}$ e $V_{e}$ sào subespaços da decomposição de Peirce $A=I \epsilon \oplus U_{e} \oplus V_{e}$ de $A$ associada ao idempotente $\epsilon$.

Já vimos que a cada polinômio $p(U, V)$ de $\mathcal{P}$ corresponde um $\mathrm{P}$-subespaço $p\left(U_{e}, V_{\epsilon}\right)$. Mas, em álgebras de Bernstein, esta correspondência nào é biunívoca. De fato, dados $p(U, V)=U V, q(U, V)=U V+(U V) V, r(U, V)=U V+(U V) V+U^{3}$, temos $p\left(U_{\epsilon}, V_{e}\right)=$ $q\left(U_{e}, V_{e}\right)=r\left(U_{e}, V_{e}\right)=U_{e} V_{e}$, visto que $U_{e}^{3},\left(U_{e} V_{e}\right) V_{e} \subseteq U_{e} V_{e}$. Exceto quando for necessário, não faremos distinção entre $p(U, V)$ e $p\left(U_{e}, V_{e}\right)$. Por exemplo, chamaremos $p(U, V)$ também de P-subespaço e escreveremos $p(U, V)=q(U, V), r(U, V) \subseteq p(U, V)$, etc.

Diremos que um P-subespaço $p(L, V)$ é invariante quanto à escolha do idempotente. ou simplesmente que é invariante. quando, para $\epsilon, f \in \operatorname{Ip}(A)$ quaisquer, tem-se $p\left(U_{\epsilon}, V_{\epsilon}\right)=$ $p\left(U_{f}, V_{f}\right)$. Se $\operatorname{dim} p\left(U_{e}, V_{\epsilon}\right)=\operatorname{dim} p\left(U_{f}, V_{f}\right)$ para quaisquer $\epsilon . f \in \operatorname{Ip}(A)$, diremos que $p(U, V)$ tem dimension incariante quanto à escolha do idempotente ou apenas que $p(U, V)$ tem dimensio invariante. Muitas vezes escreveremos $p_{\epsilon}$ no lugar de $p\left(U_{\epsilon}, V_{\epsilon}\right)$.

Tentaremos responder as seguintes questòes:

1. Quais P-subespaços de uma álgebra de Bernstein sào invariantes?

2. Quais P-subespaços têm dimensão invariante?

3. Se $p(\ell . V)$ tem dimensào invariante e dados $\epsilon \cdot f \in \operatorname{Ip}(\mathcal{A})$. existe um automorfismo de espaços vetoriais "natural" $\gamma: A \rightarrow A$ tal que $\gamma\left(p_{e}\right)=p_{f}$ ?

4. Como. a partir de um dado P-subespaço, podemos construir outros subespaços invariantes?

j. Quais P-subespaços sào ideais? 
6. Existe uma seqüência decrescente $m_{1}(U, V) \supseteq m_{2}(U, V) \supseteq \ldots \supseteq m_{k}(U . V) \supseteq \ldots$ de P-monômios tal que se $m_{r}(U, V)=0$ para algum inteiro positivo $r$ entào o núcleo de $A$ é nilpotente?

A seguir, provaremos alguns resultados que serão úteis para o próximo capítulo.

Lema 1.1 Em toda álgebra de Bernstein tem-se
(a) $U^{2 k} \subseteq V \quad$ e $\quad U^{2 k+1} \subseteq U \quad k=1,2,3, \ldots$
(b) $r \subseteq L \quad r=2,3,4, \ldots$
(c) $U^{k} L=0 \quad k=1,2,3, \ldots$

Demonstraçĩo:

(a) Esaremos induçào em $k$. Para $k=1$ temos $l^{2} \subseteq V^{*}$ e $l^{3} \subseteq L^{*}$. Se (a) vale para $k$ entào

$$
\begin{gathered}
U^{2(k+1)}=U^{2 k+2}=\left(U^{2 k} U\right) U \subseteq(V U) U \subseteq V . \\
U^{2(k+1)+1}=U^{2 k+2+1}=\left(U^{2 k+1} U\right) U \subseteq U^{3} \subseteq U .
\end{gathered}
$$

(b) Temos que $V^{2} \subseteq U$ e $U V^{2}=0$. Logo. $V^{2} \subseteq L$. Como $L$ é ideal, segue-se que $V r \subseteq L$ para todo inteiro positivo $r \geq 2$.

(c) Se $k$ é ímpar então, segue de $(a)$ e da definiçào de $L$ que

$$
U^{k} L \subseteq U L=0
$$

Se $k \geq 2$ é par então, usando a parte (a) da Proposiçào 0.1 , temos

$$
\left.U^{k} L=\left(U^{k-1} U\right) L \subseteq U^{2} L \subseteq U^{\prime} L\right)=0 .
$$

O próximo corolário segue imediatamente de $(b)$ e $(c)$ do Lema 1.1.

Corolário 1.1 Em toda álgebra de Bernstein tem-se

(a) $V^{r} \subseteq L^{r} \quad r=2,3,4, \ldots$

(b) $U^{k} V^{r}=0 \quad k=1,2,3, \ldots$ e $\quad r=2,3,4, \ldots$

As observações seguintes sào de grande utilidade.

Observação 1.1 Segue de (j) que cada P-monômio nào nulo de uma álgebra de Bernstein satisfaz uma das duas possibilidades: tstá contido $\mathrm{em} l \mathrm{l}$ ou $\epsilon$ stá contido $\mathrm{em} \mathrm{I}$. Assim. dado um P-subespaço p. existem dois P-subespaços $g \subseteq V+h \subseteq V$ tal que $p=g \neq h$. 
Observação 1.2 Se $m$ é um P-monômio com $\partial m \geq 2$ então txistem P-monômios $m_{1} \epsilon$ $m_{2}$ com $\partial m_{1}, \partial m_{2}<\partial m$ tal que $m=m_{1} m_{2}$. Usando (5), concluímos que se $m \subseteq V$ então $m_{1}, m_{2} \subseteq U$. Agora. se $m \subseteq U$ existem duas possibilidades: $m_{1} \subseteq U$ e $m_{2} \subseteq V$. ou $m_{1}$, $m_{2} \subseteq V$.

Esta fatoraçào de $m$ nào é única. Por exemplo, se $m=U\left(((U V) V) U^{2}\right)$, pela parte $(c)$ da Proposição 0.1,

$$
m=U^{3}((U V) V)=(U V)\left(U^{3} V\right)=U\left(\left(U^{3} V\right) V\right)
$$

Os P-subespaços contidos em $V$ e de ordem maior ou igual a 2 satisfazem a seguinte propriedade:

Proposição 1.1 Seja $p \subseteq V$ um P-subespaço de uma álgebra de Bernstein. Se $o(p) \geq 2$ então existe um P-subespaço $q \subseteq U$ tal que $p=U q$.

Demonstração: Suponhamos inicialmente que $p=m$ é um P-monômio com $\partial m \geq 2$. Portanto, existem P-monômios $\mu_{1}, \mu_{2} \subseteq U$ tais que $m=\mu_{1} \mu_{2}$. Mostraremos, usando indução em $\partial \mu_{1}$, que existe um P-monômio $\mu \subseteq U$ tal que $m=U \mu$. Se $\partial \mu_{1}=1$, então $m=U \mu_{2}$. A hipótese de indução é a seguinte: se $m=n q \operatorname{com} n, q \subseteq U$ e $\partial n \leq k$, então existe $\mu \subseteq U$ tal que $m=U \mu$. Suponhamos que $\partial \mu_{1}=k+1$. Se $\mu_{1} \subseteq V^{2}$, então $m=\mu_{1} \mu_{2} \subseteq V^{2} U=0$. Se $\mu_{1} \subseteq U V$, então existem P-monômios $\mu_{1}^{\prime} \subseteq U$ e $\nu \subseteq V$, tais que $\mu_{1}=\mu_{1}^{\prime} \nu$ e $\partial \mu_{1}^{\prime} \leq k$. Portanto. $m=\left(\mu_{1}^{\prime} \nu\right) \mu_{2}=\mu_{1}^{\prime}\left(\mu_{2} \nu\right)$. esta última igualdade, por (c) da Proposição 0.1. Como $\mu_{2} \nu \subseteq U$, pela hipótese de indução, existe $\mu \subseteq U$ tal que $m=U \mu$. Agora, suponhamos que $p=\sum_{i=1}^{k} m_{i}$ é um P-subespaço contido em $V$. onde $m_{i}$ são $\mathrm{P}$-monômios com $\partial m_{i} \geq 2(i=1, \ldots, k)$. Logo, existem $\mathrm{P}$-monômios $\mu_{i} \subseteq U$ tais que $m_{i}=U \mu_{i}$. Assim, escrevendo $q=\sum_{i=1}^{k} \mu_{i}$, temos $p=U q \operatorname{com} q \subseteq U$.

Os seguintes diagramas contém os $\mathrm{P}$-monômios contidos em $U$ e $V$ até grau 4 e j. respectivamente. Cada subespaço está contido em um outro imediatamente a sua esquerda e ligado a este por uma reta. As setas significam inclusões. 


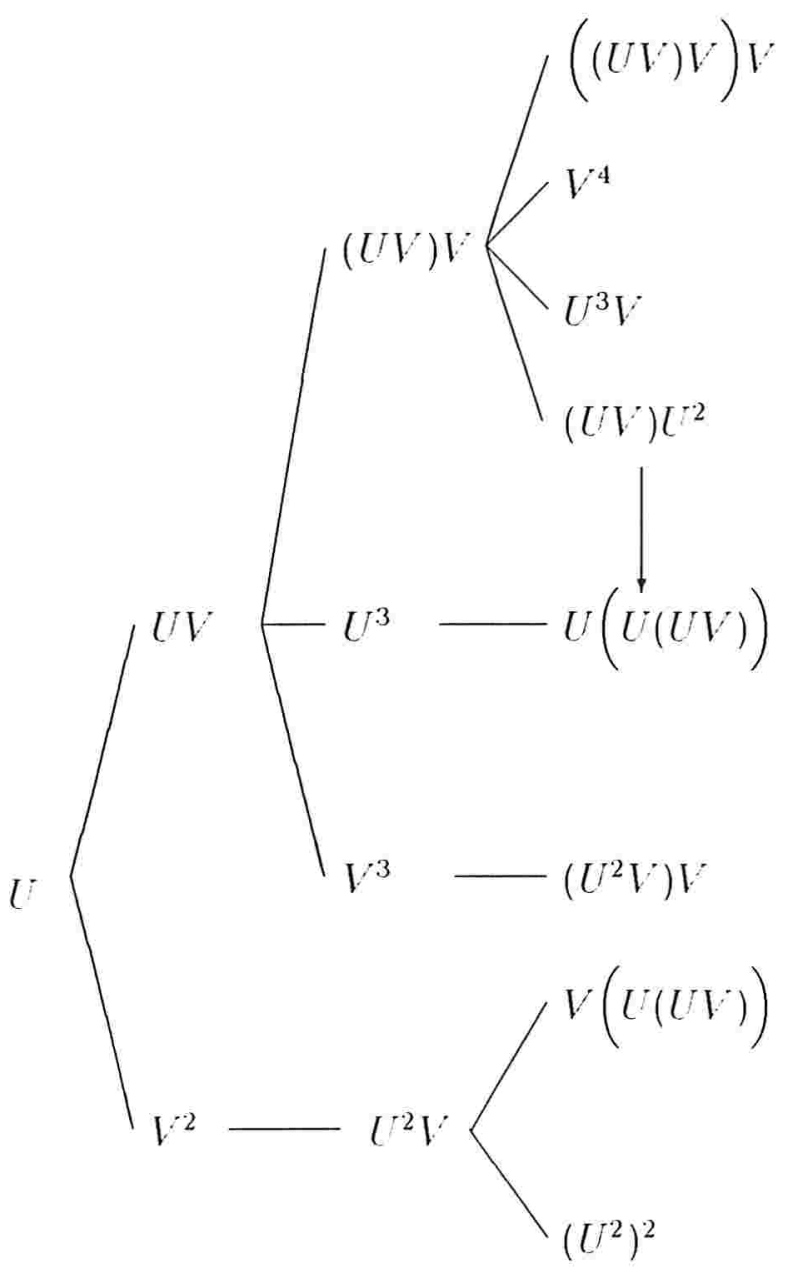

Figura 1.1: P-monômios contidos em $U$ de grau menor ou igual 4. 


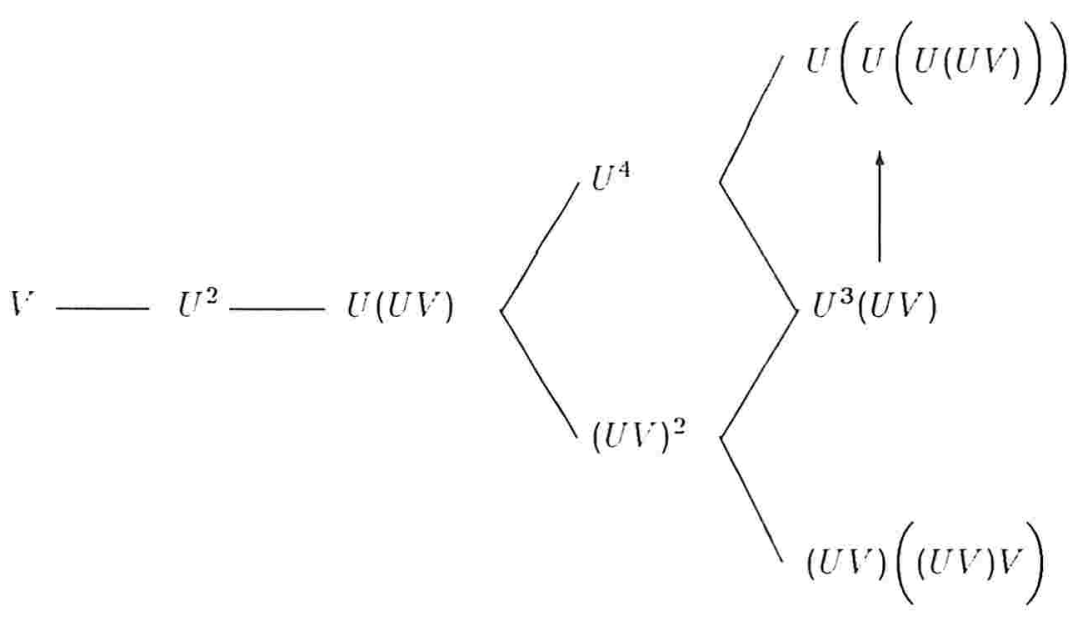

Figura 1.2: P-monômios contidos em $V$ de grau menor ou igual 5.

\subsection{Nilpotência e P-subespaços}

Seja $A=$ Ke $\oplus U \oplus V$ uma decomposição de Peirce de uma álgebra de Bernstein. Denotamos por $\operatorname{End}(A)$ a álgebra dos operadores lineares de $A$. Para cada $a \in A$ definimos $L_{a} \in \operatorname{End}(A)$ por $L_{a}(x)=a x$, para todo $x \in A$. Usamos a notação $E(V)$ para indicar a subálgebra de $\operatorname{End}(A)$ gerada pelo conjunto $\left\{L_{v} ; v \in V\right\}$.

Sejam X. $Y$ subespaços de $A$, definimos recursivamente: $Y X^{(0)}=Y$ e $Y X^{(k)}=$ $\left(Y X^{\prime(k-1)}\right) X^{\prime}$

Proposição 1.2 Existe um idempotente e de uma álgebra de Bernstein $A=K \dot{I} \oplus U \Psi V$ tal que a seqüéncia de P-monômios $U \supseteq U V \supseteq \ldots \supseteq \bigcup V(k) \supseteq V^{(j+1)} \supseteq \ldots$ estaciona $\mathrm{em}$ 0 se, e somente se, o núcleo de $A$ é nilpotente.

Demonstração: Suponhamos que $U^{\prime} V^{(k)}=0$ para algum inteiro positivo $k$. Mostremos que $E(V)^{k+1}=0$. De fato. $E(V)^{k+1}$ é gerada por elementos da forma $T=L_{v_{1}} \ldots L_{v_{k+1}}$, onde $v_{1}, \ldots, v_{k+1} \in V$. É claro que $T(\epsilon)=0$. Agora. se $u \in U$ entào $T(u) \in V^{(k+1)} U \subseteq V^{(k)} U=0$ e, se $v \in V$ entào $T(v) \in V^{k+2}=V^{(k)} V^{2} \subseteq V^{(k)} U=0$. Assim. a álgebra $E(V)$ é nilpotente. Portanto, segue do Teorema 4.7 de [1:3] que o núcleo de $A$ é nilpotente. A recíproca é evidente.

Uma conseqüência desta definiçào é que se, para algum idempotente $\epsilon$, a seqüência $U_{\epsilon} \supseteq V_{\epsilon} V_{\epsilon} \supseteq \ldots \supseteq V_{e} V_{\epsilon}^{(k)} \supseteq \zeta_{e} V_{\epsilon}^{(k+1)} \supseteq \ldots$ estaciona em 0 . entào. para todo idempotente $f$, a seqüência $l_{f} \supseteq L_{f} V_{j} \supseteq \ldots \supseteq U_{f} V_{f}^{(k)} \supseteq U_{f} V_{f}^{(k+1)} \supseteq \ldots$ também estaciona em 0 . 


\section{Capítulo 2}

\section{Invariância de $\mathbf{P}$-subespaços em álgebras de Bernstein}

Tentaremos responder as questões levantadas na página 14, exceto a última que já foi respondida no capítulo anterior. Não foi possivel dar respostas definitivas para aquelas perguntas na classe inteira das álgebras de Bernstein. Mas na subclasse das álgebras de Bernstein-Jordan, respondemos completamente a todas as questões. Em outra subclasse, que contém as álgebras de Bernstein-Jordan. demos respostas bastante satisfatórias. Também para certas famílias de P-subespaços é possível resolver tais problemas. Por exemplo, para os P-subespaços contidos em $V$, para os contidos em $U$ que satisfazem a condição $\sigma_{u}\left(g_{e}\right)=g_{f_{u}}$, para quaisquer $\epsilon \in \operatorname{Ip}(A)$ e $u \in U_{\epsilon}$. e para os que tem grau menor ou igual a 3.

\subsection{Exemplos de P-subespaços invariantes}

Nesta seçào, daremos duas maneiras de construir P-subespaços invariantes em uma álgebra de Bernstein. Com os resultados que demonstraremos na Seçào 2.5, teremos outras maneiras de fabricar P-subespaços invariantes.

Seja $A=L^{\prime} \epsilon \oplus U \oplus V$ uma decomposição de Peirce de uma álgebra de Bernstein. Já sabemos do capítulo anterior que os seguintes $\mathrm{P}$-subespaços sào invariantes:

$$
\begin{aligned}
N & =U \oplus V \\
P & =\left(U V+V^{2}\right) \oplus V \\
\tilde{U} & =U^{2} \\
\tilde{V} & =\left(U V+U^{2} V\right) \pm V
\end{aligned}
$$


É claro que $N, P, \tilde{U}, \tilde{V}$ têm dimensão invariante. Como já sabemos que $U$ e $V$ têm dimensão invariante, segue de (2.2), (2.3) e (2.4) que $U V+V^{2} \cdot U^{2}$ e $U V+U^{2} V$ também têm dimensão invariante.

A partir de P-subespaços invariantes conhecidos, podemos construir outros $\mathrm{P}$-subespaços invariantes da seguinte maneira: seja $F\left(X_{1}, \ldots, X_{k}\right)$ um polinômio sem termo independente nas variáveis comutativas e não associativas $X_{1}, \ldots, X_{k}$ e sejam $q_{1}, \ldots, q_{k}, \mathrm{P}$-subespaços invariantes de uma álgebra de Bernstein. Então $p=F\left(q_{1}, \ldots, q_{k}\right)$ é um P-subespaço invariante. De fato, se $q_{i_{e}}=q_{i_{f}}(i=1, \ldots, k)$, para quaisquer idempotentes $e$ e $f$ de $A$, então $p_{e}=F\left(q_{1_{e}}, \ldots, q_{k_{e}}\right)=F\left(q_{1}, \ldots, q_{k_{f}}\right)=p_{f}$. Assim, os seguintes P-subespaços são todos invariantes

$$
\begin{aligned}
N^{2} & =\left(U V+V^{2}\right) \oplus U^{2} \\
N^{3} & =\left((U V) V+U^{3}+V^{3}+U^{2} V\right) \oplus U(U V) \\
N P & =\left(U V+V^{2}\right) \oplus U(C V) \\
N \widetilde{U} & =\left(U V+U^{2} V\right) \oplus U^{2} \\
P \widetilde{U} & =\left(U V+U^{2} V\right) \oplus U(U V) \\
V^{3}+\tilde{U}^{2} & =\left((U V) V+U^{3}+V^{3}+U^{2} V\right) \oplus U(U V) \\
N^{3}+\tilde{U}^{2}+\tilde{V}^{2} & =\left((U V) V+U^{3}+V^{3}+V^{2}\right) \oplus U^{2} \\
V^{3}+P^{2} & =\left((U V) V+U^{3}+V^{3}+V^{2}\right) \oplus U(U V) \\
P^{2} & =\left((U V) V+V^{3}+V^{2}\right) \oplus(U V)^{2} \\
\left((\tilde{U} \tilde{V}) \tilde{V}+\tilde{V}^{3}+\tilde{U}^{2} \tilde{V}\right)+(\tilde{U} \tilde{V})^{2} & =\left((U V) V+V^{3}+l^{-2} V\right) \oplus(U V)^{2}
\end{aligned}
$$

Mostraremos adiante que os $\mathrm{P}$-subespaços listados de (2.1) a (2.12) sào todos os Psubespaços de grau menor ou igual a 3 que são invariantes em todas as álgebras de Bernstein.

\subsubsection{O P-subespaço $\grave{p}$}

A seguir apresentamos um caso particular de $F\left(q_{1}, \ldots, q_{k}\right)$. Seja $p=p(U, V)$ um elemento de $\mathcal{P}$, definimos $\tilde{p}$ como sendo o P-subespaço obtido de $p$, substituindo-se $U$ por $U^{r}+U^{2}$ e $V$ por $U V+U^{2} V+V$. isto é. $\tilde{p}=p\left(\tilde{U}_{\epsilon} \cdot \tilde{V}_{\epsilon}\right)$. Quando $p=U^{\prime}$ temos $\tilde{p}=\tilde{U}=U^{U}+U^{2}$. Analogamente se $p=V$ entào $\tilde{p}=\tilde{V}=U V+C^{-2} V+V$. Deve ficar claro que $\tilde{p}$ está associado ao polinômio $p\left(U^{\prime} \cdot V^{\prime}\right)$ e nào ao P-subespaço $p\left(l_{\epsilon}, V_{\epsilon}\right)$. Está distinção é necessária. 
pois, conforme mostra o exemplo seguinte. existem $\mathrm{P}$-subespaços que são distintos como polinômios e $p_{e}=q_{e}$ para qualquer $e \in \operatorname{Ip}(A)$. mas existe $f \in \operatorname{Ip}(A)$ tal que $\tilde{p}_{f} \neq \tilde{q}_{f}$.

Exemplo 2.1 Seja A álgebra de Bernstein tal que $\left\{e, u_{1}, u_{2}, u_{3}, u_{4}, v_{1}, v_{2}\right\}$ é uma base de.A com $U=\left\langle u_{1} \cdot u_{2}, u_{3}, u_{4}\right\rangle, V=\left\langle v_{1}, v_{2}\right\rangle$ e tábua de multiplicaçào do núcleo dada por

\begin{tabular}{l||llll|ll} 
& $u_{1}$ & $u_{2}$ & $u_{3}$ & $u_{4}$ & $v_{1}$ & $v_{2}$ \\
\hline \hline$u_{1}$ & & & & & $u_{2}$ & $u_{3}$ \\
$u_{2}$ & & & & & & $u_{4}$ \\
$u_{3}$ & & & & & $-u_{4}$ & \\
$u_{4}$ & & & & & & \\
\hline$v_{1}$ & & & & & \\
$v_{2}$ & & & & & &
\end{tabular}

Esta álgebra é a do exemplo 7 de [15]. É uma álgebra de Bernstein-Jordan. Logo, $V_{f}^{2}=0$ para todo $f \in \operatorname{Ip}(A)$. Assim, temos também que $l_{f}^{2} V_{f}=0$ para todo $f \in \operatorname{Ip}(A)$. Sejam $p=V^{2}$ e $q=U^{2} V$. Como polinômios em $U$ e $V$, temos que $p$ e $q$ são distintos, mas

$$
p_{f}=q_{f}=0
$$

para todo $f \in \operatorname{Ip}(A)$. Agora

$$
\begin{aligned}
& \tilde{p}_{e}=\tilde{V}_{e}^{2}=\left(U_{e} V_{e}\right) V_{e}=\left\langle u_{4}\right\rangle \\
& \tilde{q}_{e}=\tilde{U}_{e}^{2} \tilde{V}_{e}=\left(U_{e}+U_{e}^{2}\right)^{2}\left(U_{e} V_{e}+V_{e}\right)=0
\end{aligned}
$$

Portanto, $\tilde{p}_{e} \neq \tilde{q}_{\epsilon}$.

O P-subespaço $\tilde{p}$ satisfaz as seguintes propriedades:

Proposição 2.1 Para qualquer P-subespaço p de uma álgebra de Bernstein, tem-se

(a) $\tilde{p}$ é invariante;

(b) $p \subseteq \tilde{p}$ :

(c) $\tilde{\tilde{p}}=\tilde{p}$. 
Demonstração: Como $\tilde{U}$ e $\tilde{V}$ são invariantes. temos que $p(\tilde{U}, \tilde{V})$ é invariante. Agora, $U \subseteq$ $\tilde{U}$ e $V \subseteq \tilde{V}$. Logo, $p(U, V) \subseteq p(\tilde{U}, \tilde{V})$. Assim, provamos $(a)$ e $(b)$. Para mostrar $(c)$, observemos inicialmente que $\tilde{\widetilde{U}}=\tilde{U}$ e $\tilde{\tilde{V}}=\tilde{V}$. De fato, segue de (5) que

$$
\begin{aligned}
\tilde{\widetilde{U}} & =U+U^{2}+\left(U+U^{2}\right)^{2} \\
& =U+U^{2} \\
\tilde{\tilde{V}} & =\left(U+U^{2}\right)\left(U V+U^{2} V+V\right)+\left(U+U^{2}\right)^{2}\left(U V+U^{2} V+V\right)+U V+U^{2} V+V \\
& =U V+U^{2} V+V .
\end{aligned}
$$

Agora, por definiçào, temos que $\tilde{\tilde{p}}=\tilde{p}(\tilde{U}, \tilde{V})=p(\tilde{\widetilde{U}}, \tilde{\widetilde{V}})=p(\tilde{U} \cdot \tilde{V})=p$.

$\mathrm{Na}$ verdade. demonstra-se de maneira análoga o seguinte fato mais geral que a parte $(c)$ da proposiçào anterior: dados $q_{1}, \ldots q_{k}$ P-subespaços invariantes e $p=F\left(q_{1}, \ldots, q_{k}\right)$ como antes, se $\tilde{q}_{1}=q_{1}, \ldots, \tilde{q}_{k}=q_{k}$ então $\tilde{p}=p$.

Assim como $\tilde{\widetilde{U}}=\tilde{U}$ e $\tilde{\tilde{V}}=\tilde{V}$. temos também que $\widetilde{N}=N$ e $\tilde{P}=P$. Logo, qualquer P-subespaço $p$ listado de (2.1) a (2.14) satisfaz $\tilde{p}=p$. Portanto, todos os P-subespaços desta lista sào obtidos a partir de um polinômio de duas variáveis e dos P-subespaços $\widetilde{U}$ e $\tilde{V}$.

\subsubsection{O P-subespaço $S_{k, r}$}

Seja $A=K e \oplus U \oplus V$ uma álgebra de Bernstein e sejam $k$ e $r$ inteiros positivos. O P-subespaço $S_{k, r}$ é definido como sendo a soma de todos os P-monômios contidos em $U$ de grau $k$ mais a soma de todos os P-monômios contidos em $V$ de grau $r$. Usaremos as seguintes notações:

$M_{k, U}$ é o conjunto dos P-monômios de $A$, contidos em $\ell^{\prime}$. com grau igual a $k$.

$M_{k, V}$ é o conjunto dos P-monômios de $A$, contidos em $V$, com grau igual a $k$.

$$
S_{k, U}=\sum_{m \in M_{k, U}} m, \quad S_{k, V}=\sum_{n \in M_{k, V}} n .
$$

Assim, $S_{k, r}=S_{k, U} \oplus S_{r, V}$.

Temos as seguintes cadeias encaixantes

$$
\begin{aligned}
& S_{1, U} \supseteq \ldots \supseteq S_{k, L} \supseteq S_{k+1, U} \supseteq \ldots \\
& S_{1, V} \supseteq \ldots \supseteq S_{k, V} \supseteq S_{k+1, V} \supseteq \ldots
\end{aligned}
$$


De fato, segue de (5) que cada P-monômio $m_{1} \subseteq U$ (resp. $n_{1} \subseteq V$ ) de grau $k+1$ está contido em um P-monômio $m_{2} \subseteq U$ (resp. $n_{2} \subseteq V$ ) de grau $k$.

A proposição seguinte nos diz para que valores de $k$ e $r$ o P-subespaço $S_{k, r}$ é invariante.

Proposição 2.2 Para qualquer inteiro positivo $k$, os P-subespaços $S_{k+1, k}, S_{k, k}$ e $S_{k, k+1}$ sào ideais e são invariantes quanto à escolha do idempotente.

Demonstração: Seja At uma álgebra de Bernstein. Mostremos que $S_{k, k+1}$ é um ideal. A demonstração de que $S_{k+1, k}$ e $S_{k, k}$ são ideais é análoga. Temos que

$$
\epsilon S_{k, k+1}=S_{k, U} \subseteq S_{k, k+1}
$$

Observemos que $I S S_{r . L} \subseteq S_{r+1, V}, U S_{r . V} \subseteq S_{r+1, l} V S_{r . V} \subseteq S_{r+1, U}$ e $V S_{r . V} \subseteq S_{r+1, l^{*} \text { para }}$ todo inteiro positivo $r$. Portanto.

$$
\begin{aligned}
& U S_{k, k+1}=U S_{k, U}+U S_{k+1, V} \subseteq S_{k+1, V}+S_{k+2, U} \subseteq S_{k, U}+S_{k+1, V}=S_{k, k+1} \\
& V S_{k, k+1}=V S_{k, U}+V S_{k+1, V}=S_{k+1, U}+S_{k+2, U} \subseteq S_{k, U} \subseteq S_{k, k+1}
\end{aligned}
$$

Assim, $S_{k, k+1}$ é ideal.

Seja $m$ um P-monômio de grau $r$ e sejam $\epsilon$ e $f$ dois idempotentes quaisquer de $A$. Existe $u \in U_{e}$ tal que $f=\epsilon+u+u^{2}$. Entào segue de (1S) e (19) e pela definiçào de subespaço monomial, dada na página 13 , que

$$
m\left(U_{f}, V_{f}\right) \subseteq m\left(U_{\epsilon}, V_{\epsilon}\right)+S_{r+1, U_{e}}+S_{r+1, V_{e}}
$$

Suponhamos agora que $m$ é um P-monômio de $S_{k . l}$. Usando (2.1T) obtemos

$$
m\left(I_{f}, V_{f}\right) \subseteq m\left(U_{e}, V_{e}\right)+S_{k+1, l_{e}}+S_{k+1, V_{e}} \subseteq S_{k, U_{e}} \pm S_{k+1 . V_{e}}
$$

Portanto. $S_{k \cdot l_{j}} \subseteq S_{k \cdot l_{e}} \oplus S_{k+1, V_{e}}$. Da mesma maneira. mostra-se que $S_{k+1, V_{f}} \subseteq S_{k \cdot l_{e}}=$ $S_{k+1, V_{e}}$. Assim. $S_{k, l^{\prime}}, S_{k+1, V_{j}} \subseteq S_{k, l_{e}} \pm S_{k+1, V_{e}}$. Como esta inclusão vale para quaisquer dois idempotentes de $A$, temos que $S_{k, U_{e}} \Phi S_{k+1, V_{e}}=S_{k, L_{1}}=S_{k+1, V_{f}}$. Logo, $S_{k, k+1}$ é invariante. De maneira análoga. mostra-se que $S_{k+1, k}$ e $S_{k, k}$ também sào invariantes.

Os P-subespaços $S_{k+1, k}, S_{k, k}$ e $S_{k, k+1}$ satisfazem a propriedade

$$
\begin{aligned}
S_{k+1, k} & =\widetilde{S}_{k+1, k} \\
S_{k, k} & =\widetilde{S}_{k, k} \\
S_{k, k+1} & =\widetilde{S}_{k, k+1}
\end{aligned}
$$


De fato, segue da definição de $\tilde{p}$ e de $S_{k, r}$ que $\widetilde{S}_{k, k+1} \subseteq S_{k, k+1}+S_{k+1, k+2}$. De (2.15) e (2.16), temos que $S_{k+1, k+2} \subseteq S_{k, k+1}$. Logo $\widetilde{S}_{k, k+1} \subseteq S_{k, k+1}$. Da Proposição 2.1, segue-se que $S_{k, k+1} \subseteq \widetilde{S}_{k, k+1}$. Portanto. $S_{k+1, k}=\widetilde{S}_{k, k+1}$. As outras igualdades são demonstradas de maneira análoga.

Utilizaremos agora o $\mathrm{P}$-subespaço $S_{2, L^{-}}^{\prime}$ para mostrar que nem todo ideal do núcleo de uma álgebra de Bernstein é um P-subespaço. Com efeito, consideremos o ideal $L$ e a álgebra de Bernstein $A$ com base $\left\{e, u_{1}, u_{2}, u_{3}, v_{1}, v_{2}\right\}$, decomposição de Peirce $A=K \epsilon \oplus\left\langle u_{2}, u_{2}, u_{3}\right\rangle \oplus$ $\left\langle v_{1}, v_{2}\right\rangle$ e tábua de multiplicaçào do núcleo dada por

\begin{tabular}{c||ccc|cc} 
& $u_{1}$ & $u_{2}$ & $u_{3}$ & $v_{1}$ & $v_{2}$ \\
\hline \hline$u_{1}$ & $v_{1}$ & & & & \\
$u_{2}$ & & & & & $u_{2}$ \\
$u_{3}$ & & & & & \\
\hline$v_{1}$ & & & & & \\
$v_{2}$ & & & & &
\end{tabular}

temos $U \supset L \supset S_{2, U}$ (contém e é diferente), pois $U=\left\langle u_{1}, u_{2}, u_{3}\right\rangle . L=\left\langle u_{2}, u_{3}\right\rangle$ e $S_{2, U}=$ $U V+V^{2}=\left\langle u_{2}\right\rangle$. Observemos que $S_{2, U}$ contém todos os $\mathrm{P}$-subespaços de ordem maior ou igual a 2 contidos em $U$. Como $L \supset S_{2, L}$, temos que $L$ não é um $\mathrm{P}$-subespaço de ordem maior ou igual a 2. Além disso, $U \supset L$. Logo, $L$ nào é um P-subespaço de ordem 1. Portanto, $L$ nào é P-subespaço.

\subsection{Exemplos de P-subespaços não invariantes}

Nesta seção. daremos exemplos de $\mathrm{P}$-subespaços que não sào invariantes e de $\mathrm{P}$ subespaços que nào têm dimensào invariante. Para isso, estudaremos três exemplos de álgebras de Bernstein. Em cada um deles. (A.w) é uma álgebra de Bernstein sobre um corpo $K \cdot\left\{\epsilon, u_{1}, \ldots, u_{r}, v_{1}, \ldots, v_{s}\right\}$ é uma base de $A \cdot \operatorname{com} U=\left\langle u_{1}, \ldots u_{r}\right\rangle, V=\left\langle v_{1}, \ldots, v_{s}\right\rangle$, $\omega(\epsilon)=1$ e $\omega\left(u_{i}\right)=\omega\left(v_{j}\right)=0(i=1, \ldots, r, j=1, \ldots s)$. Nào faremos os cálculos para mostrar que realmente as álgebras são de Bernstein e cada P-subespaço será determinado através da exibiçào de uma base.

Exemplo 2.2 $A=\left\langle e, u_{1}, u_{2}, u_{3}, u_{4}, u_{5}, u_{6}, v_{1}, v_{2}, v_{3}\right\rangle$, com tábua de multiplicação dada por $\epsilon u_{i}=\frac{1}{2} u_{i}, \epsilon v_{j}=0 \epsilon$ 


\begin{tabular}{l||cccccc|ccc} 
& $u_{1}$ & $u_{2}$ & $u_{3}$ & $u_{4}$ & $u_{5}$ & $u_{6}$ & $v_{1}$ & $v_{2}$ & $v_{3}$ \\
\hline \hline$u_{1}$ & & & & $v_{2}$ & & & $u_{3}$ & & \\
$u_{2}$ & & $v_{2}$ & $-v_{2}$ & & & & $u_{4}$ & & \\
$u_{3}$ & & $-v_{2}$ & & & & & & & \\
$u_{4}$ & $v_{2}$ & & & & & & & & \\
$u_{5}$ & & & & & & & & & $u_{6}$ \\
$u_{6}$ & & & & & & & & & \\
\hline$v_{1}$ & $u_{3}$ & $u_{4}$ & & & & & & & \\
$v_{2}$ & & & & & & & $u_{5}$ & & \\
$v_{3}$ & & & & & $u_{6}$ & & & &
\end{tabular}

Para os idempotentes $\epsilon$ e $f=e+u_{2}+v_{2}$, temos

$$
\begin{array}{ll}
U_{e}=\left\langle u_{1}, u_{2}, u_{3}, u_{4}, u_{5}, u_{6}\right\rangle & U_{f}=\left\langle u_{1}, u_{2}+2 v_{2}, u_{3}-2 v_{2}, u_{4}, u_{5}, u_{6}\right\rangle \\
V_{\epsilon}=\left\langle v_{1}, v_{2}, u_{3}\right\rangle & V_{f}=\left\langle v_{1}-2 u_{4}-2 u_{5}, v_{2}, v_{3}\right\rangle \\
U_{e} V_{e}=\left\langle u_{3}, u_{4}, u_{6}\right\rangle & U_{f} V_{f}=\left\langle u_{3}-2 v_{2}, u_{4}, u_{5}, u_{6}\right\rangle \\
V_{e}^{2}=\left\langle u_{5}\right\rangle & V_{f}^{2}=\left\langle u_{5}, u_{6}\right\rangle \\
\left(U_{e} V_{e}\right) V_{e}=0 & \left(U_{f} V_{f}\right) V_{f}=\left\langle u_{5}, u_{6}\right\rangle \\
U_{e}^{3}=0 & U_{f}^{3}=0 \\
V_{e}^{3}=\left\langle u_{6}\right\rangle & V_{f}^{3}=\left\langle u_{6}\right\rangle \\
U_{e}^{2} V_{\epsilon}=\left\langle u_{5}\right\rangle & U_{f}^{2} V_{f}=\left\langle u_{5}\right\rangle \\
U_{e} V_{e}+V_{e}^{2}=\left\langle u_{3}, u_{4}, u_{5}, u_{6}\right\rangle & U_{f} V_{f}+V_{f}^{2}=\left\langle u_{3}-2 v_{2}, u_{4}, u_{5}, u_{6}\right\rangle \\
U_{\epsilon} V_{e}+U_{e}^{2} V_{\epsilon}=\left\langle u_{3}, u_{4}, u_{5}, u_{6}\right\rangle & U_{f} V_{f}+V_{f}^{2} V_{f}=\left\langle u_{3}-2 v_{2}, u_{4}, u_{5}, u_{6}\right\rangle \\
V_{\epsilon}^{2}+U_{\epsilon}^{3}=\left\langle u_{5}\right\rangle & V_{f}^{2}+V_{f}^{3}=\left\langle u_{5}, u_{6}\right\rangle \\
\left(U_{\epsilon} V_{\epsilon}\right) V_{e}+V_{\epsilon}^{2}=\left\langle u_{5}\right\rangle & \left(U_{f} V_{f}\right) V_{f}+V_{f}^{2}=\left\langle u_{5}, u_{6}\right\rangle \\
U_{\epsilon}^{2} V_{e}+\left(U_{\epsilon} V_{\epsilon}\right) V_{\epsilon}=\left\langle u_{5}\right\rangle & U_{f}^{2} V_{f}+\left(U_{f} V_{f}\right) V_{f}=\left\langle u_{5}, u_{6}\right\rangle \\
V_{e}^{2}+C_{\epsilon}^{-3}+\left(U_{\epsilon} V_{\epsilon}\right) V_{\epsilon}=\left\langle u_{5}\right\rangle & V_{f}^{2}+\left(U_{f}^{3}+\left(U_{f} V_{f}\right) V_{f}=\left\langle u_{5}, u_{6}\right\rangle\right. \\
\left(U_{e} V_{\epsilon}\right) V_{\epsilon}+V_{\epsilon}^{3}=\left\langle u_{6}\right\rangle & \left(U_{f} V_{f}\right) V_{f}+V_{f}^{3}=\left\langle u_{5}, u_{6}\right\rangle \\
U_{\epsilon}^{3}+U_{\epsilon}^{2} V_{\epsilon}+\left(U_{\epsilon} V_{\epsilon}\right) V_{\epsilon}=\left\langle u_{5}\right\rangle & U_{f}^{3}+U_{f}^{2} V_{f}+\left(U_{f} V_{f}\right) V_{f}=\left\langle u_{5}, u_{6}\right\rangle
\end{array}
$$


Portanto, os P-subespaços

$$
\begin{array}{ll}
U & U V+V^{2} \\
V & U V+U^{2} V
\end{array}
$$

embora tenham dimensão invariante, conforme observamos na seção anterior, não são invariantes. Para os P-subespaços

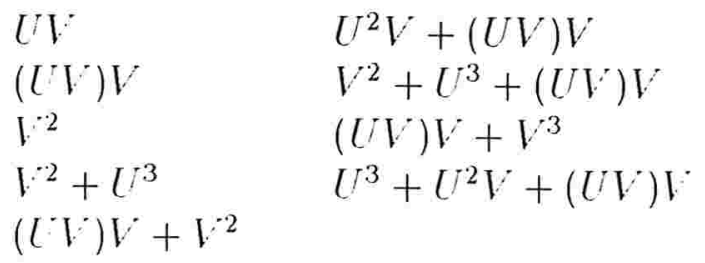

a dimensào depende do idempotente escolhido para decompor A.

Exemplo 2.3 $A=\left\langle\epsilon, u_{1}, u_{2}, u_{3}, v_{1}, v_{2}, v_{3}\right\rangle$. com tábua de multiplicaçào dada por $e u_{i}=\frac{1}{2} u_{i}$, $\epsilon v_{i}=0 \epsilon$

\begin{tabular}{l||lll|lll} 
& $u_{1}$ & $u_{2}$ & $u_{3}$ & $v_{1}$ & $v_{2}$ & $v_{3}$ \\
\hline \hline$u_{1}$ & $v_{1}$ & $v_{3}$ & & & & \\
$u_{2}$ & $v_{3}$ & $v_{2}$ & & & & \\
$u_{3}$ & & & & & & \\
\hline$v_{1}$ & & & & $2 u_{3}$ & \\
$v_{2}$ & & & & $2 u_{3}$ & & \\
$v_{3}$ & & & & & & $-u_{3}$
\end{tabular}

Para os idempotentes $\epsilon$ e $f=\epsilon+u_{1}+u_{2}+v_{1}+v_{2}+2 v_{3}$ temos

$$
\begin{aligned}
& U_{\epsilon}^{3}=U_{e}^{3}+\left(U_{e} V_{\epsilon}\right) V_{\epsilon}=U_{\epsilon}^{3}+V_{\epsilon}^{3}=U_{e}^{3}+\left(U_{\epsilon} V_{\epsilon}\right) V_{\epsilon}+V_{\epsilon}^{3}=0 \\
& U_{f}^{3}=U_{f}^{3}+\left(U_{f} V_{f}\right) V_{f}=U_{f}^{3}+V_{f}^{3}=U_{f}^{3}+\left(U_{f} V_{f}\right) V_{f}+V_{f}^{3}=\left\langle u_{3}\right\rangle
\end{aligned}
$$

Portanto, a dimensào dos $\mathrm{P}$-subespaços

$$
\begin{aligned}
& l^{-3} \\
& U^{3}+I^{3} \\
& L^{3}+(L \mathrm{~V}) \mathrm{V} \\
& L^{33}+\left(C^{\circ}\right) I^{\circ}+I^{-3}
\end{aligned}
$$

depende da escolha do idempotente. 
Exemplo 2.4 $A=\left\langle\epsilon, u_{1}, u_{2}, u_{3}, u_{4}, u_{5}, u_{6}, v_{1}, v_{2}, v_{3}, v_{4}\right\rangle$, com a seguinte tábua de multiplicação: $\epsilon u_{i}=\frac{1}{2} u_{i}, e v_{j}=0 \epsilon$

\begin{tabular}{l||cccccc|cccc} 
& $u_{1}$ & $u_{2}$ & $u_{3}$ & $u_{4}$ & $u_{5}$ & $u_{6}$ & $v_{1}$ & $v_{2}$ & $v_{3}$ & $v_{4}$ \\
\hline \hline$u_{1}$ & $v_{2}$ & & $v_{3}$ & $-v_{4}$ & & & $u_{2}$ & & & $\frac{1}{2} u_{5}$ \\
$u_{2}$ & & & $v_{4}$ & & & & & & $-\frac{1}{2} u_{5}$ & \\
$u_{3}$ & $v_{3}$ & $v_{4}$ & & & & & $u_{4}$ & & & \\
$u_{4}$ & $-v_{4}$ & & & & & & & $u_{5}$ & & \\
$u_{5}$ & & & & & & $u_{6}$ & & & \\
$u_{6}$ & & & & & & & & & \\
\hline$v_{1}$ & $u_{2}$ & & $u_{4}$ & & $u_{6}$ & & & \\
$v_{2}$ & & & & $u_{5}$ & & & & \\
$v_{3}$ & & $-\frac{1}{2} u_{5}$ & & & & & & & & \\
$v_{4}$ & $\frac{1}{2} u_{5}$ & & & & & & & & &
\end{tabular}

Usando os idempotentes $\epsilon$ e $f=\epsilon+u_{2}+u_{3}+2 v_{4}$, obtemos

$$
\begin{aligned}
& V_{e}^{3}=U_{\epsilon}^{2} I_{\epsilon}=V_{e}^{2}+V_{e}^{3}=U_{\epsilon}^{2} V_{e}+V_{\epsilon}^{3}=0 \\
& V_{\epsilon}^{2}+U_{\epsilon}^{3}+V_{\epsilon}^{3}=I_{e}^{3}+U_{e}^{2} V_{e}+V_{e}^{3}=U_{\epsilon}^{3}+U_{\epsilon}^{2} V_{e}=\left\langle u_{5}\right\rangle \\
& V_{f}^{3}=\left\langle u_{6}\right\rangle \\
& U_{f}^{2} V_{f}=V_{f}^{2}+V_{f}^{3}=U_{f}^{2} V_{f}+V_{f}^{3}=V_{f}^{2}+U_{f}^{3}+V_{f}^{3}= \\
& \quad U_{f}^{3}+U_{f}^{2} V_{f}+V_{f}^{3}=U_{f}^{3}+U_{f}^{-2} V_{f}=\left\langle u_{5}, u_{6}\right\rangle
\end{aligned}
$$

Assim. os seguintes P-subespaços nào têm dimensào invariante:

$$
\begin{aligned}
& V^{3} \\
& U^{2} V \\
& V^{2}+V^{3} \\
& U^{2} V+V^{3}
\end{aligned}
$$

$$
\begin{aligned}
& V^{2}+I^{-3}+V^{3} \\
& U^{3}+I^{-2} V+V^{3} \\
& U^{3}+I^{-2} V
\end{aligned}
$$

O exemplo 2.3 está em [\$]. Há também outros exemplos em [\$] que mostram que os

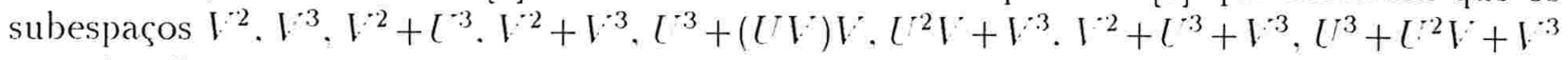
não têm dimensào invariante. Estes casos estão incluíclos nos exemplos 2.2 e 2.4 que foram feitos a partir de dois exemplos que A. Grishikov construiu para mostrar que os subespaços $U^{2} V$ e $(I V) V$ nào têm dimensòes invariantes. 


\subsection{Invariância em álgebras de Bernstein satisfazendo $U^{2} V=0$ e $(u v) v=0$}

Primeiro mostraremos que a condição (as duas igualdades juntas)

$$
\left\{\begin{array}{l}
U^{2} V=0 \\
(u v) v=0 \text { para quaisquer } u \in U \text { e } v \in V
\end{array}\right.
$$

não depende do idempotente escolhido. Assim. (2.18) determina uma subclasse de álgebras de Bernstein. Provaremos que nesta subclasse todos os P-subespaços têm dimensào invariante.

Segue de $l^{-2} V \subseteq V^{2}$ e da Proposição 0.3 que a subclasse determinada por (2.18) contém as álgebras de Bernstein-.Jordan. Mas nem toda álgebra de Bernstein que satisfaz (2.18) é uma álgebra de Bernstein-Jordan. Por exemplo. a álgebra de Bernstein $A=K e=U-V$, onde $U=\langle u\rangle$ e $V=\langle v\rangle$, com tábua de multiplicaçào dada por $\epsilon^{2}=e, e u=\frac{1}{2} u, v^{2}=u$ e os outros produtos nulos. Temos que $L^{2} V=0$, pois $U^{2}=0$ e, para quaisquer $u \in U$ e $v \in V$, temos $(u v) v=0$, visto que $U V=0$. Entretanto, A não é de Bernstein-Jordan. uma vez que $V^{2} \neq 0$.

Observação 2.1 As álgebras tratadas nesta seçào satisfazem as identidades (27) € (2S).

Seja $A=L^{\circ} \oplus \oplus U_{e} \oplus V_{e}$ uma álgebra de Bernstein. Para qualquer $u_{0} \in U_{e}$, a função linear $\psi: U_{\epsilon} \rightarrow U_{\epsilon}$ definida por

$$
\psi(u)=u-2 u_{0}^{2} u
$$

é um automorfismo de espaço vetorial. De fato, basta mostrar que $\psi$ é injetora. Seja $u \in I_{\epsilon}$ tal que $\psi(u)=0$. Logo, $u=2 u_{0}^{2} u$. Usando $(7),(12)$ e (15) obtemos as equaçòes $u=-4 u_{0}\left(u_{0} u\right)$ e $u_{0} u=2 u_{0}\left(u_{0}^{2} u\right)=-2 u u_{0}^{3}=0$ de onde segue que $u=0$.

Seja agora $f=\epsilon+u_{0}+u_{0}^{2}$ um outro idempotente de $A$ e sejam $\sigma: U_{e} \rightarrow U_{f}$ e $\tau: V_{\epsilon} \rightarrow V_{f}$ os isomorfismos de espaços vetoriais em (20) e (21). A próxima proposição nos fornece quatro identidades envolvendo $\sigma, \tau$ e $\psi$. quando $t$ satisfaz (2.18).

Proposição 2.3 Se em uma álgebra de Bernstein triste um idempotente e tal que $L_{\epsilon}^{* 2} V_{\epsilon}=0$ $e(u v) v=0$. para quaisquer $u \in U_{\epsilon} \in v \in V_{\epsilon}$. entào

(a) $\sigma\left(u_{1}\right) \sigma\left(u_{2}\right)=\tau\left(u_{1} u_{2}\right)$ :

(b) $\sigma(u) \tau(r)=\sigma(\imath \cdot(u) v)$ : 
(c) $\tau\left(v_{1}\right) \tau\left(v_{2}\right)=\sigma\left(v_{1} v_{2}\right)=v_{1} v_{2}$;

(d) $\psi(\psi(u) v)=u v$.

Demonstração: Segue de (3) e (13) que a equaçào (a) é verdadeira para qualquer álgebra de Bernstein:

$$
\begin{aligned}
\sigma\left(u_{1}\right) \sigma\left(u_{2}\right) & =u_{1} u_{2}+2 u_{1}\left(u_{0} u_{2}\right)+2\left(u_{0} u_{1}\right) u_{2}+4\left(u_{0} u_{1}\right)\left(u_{0} u_{2}\right) \\
& =u_{1} u_{2}-2 u_{0}\left(u_{1} u_{2}\right)-2 u_{0}^{2}\left(u_{1} u_{2}\right) \\
& =\tau\left(u_{1} u_{2}\right)
\end{aligned}
$$

Para provar (b) calculemos $\sigma(u) \tau(v)=u v-2 u\left(u_{0} v\right)+2\left(u_{0} u\right) v-4\left(u_{0} u\right)\left(u_{0} v\right)$. Agora (3), (15) e $U_{\epsilon}^{2} V_{e}=0$ implicam que $\sigma(u) \tau(v)=u v+2 u_{0}(u v)+2 u_{0}^{2}(u v)$. Por outro lado,

$$
\begin{aligned}
\sigma(\psi(u) v) & =\sigma\left(u v-2\left(u_{0}^{2} u\right) v\right) \\
& =u v-2\left(u_{0}^{2} u\right) v+2 u_{0}(u v)-4 u_{0}\left(\left(u_{0}^{2} u\right) v\right)
\end{aligned}
$$

Mas

$$
u_{0}\left(\left(u_{0}^{2} u\right) v\right)=0
$$

em qualquer álgebra de Bernstein. De fato, usando (12), (15) e (16) temos

$$
\begin{aligned}
u_{0}\left(\left(u_{0}^{2} u\right) v\right) & =-\left(u_{0}^{2} u\right)\left(u_{0} v\right) \\
& =2\left(u_{0}\left(u_{0} u\right)\right)\left(u_{0} v^{\prime}\right) \\
& =0
\end{aligned}
$$

Assim (28) implica (b). Mostremos agora (c). Segue da hipótese que

$$
\tau\left(v_{1}\right) \tau\left(v_{2}\right)=v_{1} v_{2}-2 v_{1}\left(u_{0} v_{2}\right)-2\left(u_{0} v_{1}\right) v_{2}+4\left(u_{0} v_{1}\right)\left(u_{0} v_{2}\right)
$$

Portanto, (16) e (28) implicam em $\tau\left(v_{1}\right) \tau\left(v_{2}\right)=v_{1} v_{2}$. Usando (14) temos $u_{0}\left(v_{1} v_{2}\right)=0$. Logo.

$$
\begin{aligned}
\tau\left(v_{1}\right) \tau\left(v_{2}\right) & =v_{1} v_{2}+2 u_{0}\left(v_{1} v_{2}\right) \\
& =\sigma\left(v_{1} v_{2}\right)
\end{aligned}
$$


Finalmente. segue de (28) que

$$
\begin{aligned}
\psi(\psi(u) v) & =\psi\left(u v-2\left(u_{0}^{2} u\right) v\right) \\
& =u v+4 u_{0}^{2}\left(\left(u_{0}^{2} u\right) v\right)
\end{aligned}
$$
Agora, de (12) e (2.20) obtemos $u_{0}^{2}\left(\left(u_{0}^{2} u\right) v\right)=-2 u_{0}\left(u_{0}\left(\left(u_{0}^{2} u\right) v\right)\right)=0$. Assim, $\psi(\psi(u) v)=$
$u v$.

A invariância de (2.18) é conseqüência do próximo corolário.

Corolário 2.1 Em uma álgebra de Bernstein A. as condições seguintes sio equivalentes:

(a) Existe um idempotente $e$ de $A$, tal que $L_{\epsilon}^{-2} V_{\epsilon}=0 \in(u v) v=0$, para todo $u \in L_{\epsilon}^{*} \epsilon$ $v \in V_{\epsilon}$ :

(b) Para todo idempotente e de $A$, tem-se $U_{e}^{2} V_{\epsilon}=0$ e (uv)v $=0$, para quaisquer $u \in U_{\epsilon} e$ $v \in V_{\epsilon}$.

Demonstraçaio: Provaremos que (a) implica (b). Seja $f=\epsilon+u_{0}+u_{0}^{2} \in \operatorname{Ip}(A) \operatorname{com} u_{0} \in U_{\epsilon}$. Como $\sigma$ e $\tau$ são isomorfismos de espaços vetoriais, $\left[T_{f}^{2} V_{f}=\left\langle\left(\sigma\left(u_{1}\right) \sigma\left(u_{2}\right)\right) \tau(v) ; u_{1} \cdot u_{2} \in\right.\right.$ $\left.U_{\epsilon}, v \in V_{\epsilon}\right\rangle$. Mas $\left(\sigma\left(u_{1}\right) \sigma\left(u_{2}\right)\right) \tau(v)=\tau\left(u_{1} u_{2}\right) \tau(v)=\left(u_{1} u_{2}\right) v=0$. Além disso,

$$
\begin{aligned}
(\sigma(u) \tau(v)) \tau(v) & =\sigma(u(u) v) \tau(v) \\
& =\sigma(\psi(\psi(u) v) v) \\
& =\sigma((u v) v) \\
& =0
\end{aligned}
$$

Faremos uso das seguintes identidades envolvendo subespaços do núcleo de $A$.

Corolário 2.2 Sejam $X, X_{1}, X_{2} \subseteq U \in W . W_{1}$. $W_{2} \subseteq V$ subespaços de uma álgebra de Bernstein que satisfaz (2.18). Entäo

(a) $\left(X W_{1}\right) W_{2}=\left(X W_{2}\right) W_{1}$ :

(b) $\sigma\left(X_{1}\right) \sigma\left(X_{2}\right)=\tau\left(X_{1} X_{2}\right)$;

(c) $\sigma\left(X^{\prime}\right) \tau\left(W^{\circ}\right)=\sigma\left(\iota^{\prime}\left(X^{\prime}\right) W^{\circ}\right)$ : 
(d) $\tau\left(W_{1}\right) \tau\left(W_{2}\right)=\sigma\left(W_{1} W_{2}\right)$

Demonstração: A parte (a) segue de (28). A Proposição 2.3 implica (b), (c) e (d).

O lema seguinte é evidente a partir da figura 1.2.

Lema 2.1 Seja e um idempotente de uma álgebra de Bernstein tal que $U_{e}^{2} V_{e}=0$. Se $m \subseteq V^{2}$ é um P-monômio com $\partial m \geq 3$ então $m\left(U_{e}, V_{e}\right)=0$.

Demonstração: Se $m \subseteq V^{2}$ e $\partial m \geq 3$ então $m \subseteq U^{2} V$. De fato. escrevamos $m=\nu_{1} \nu_{2}$, onde $\nu_{1}$ e $\nu_{2}$ são P-monômios com $\partial \nu_{1} \geq 2$. Assim, existem P-monômios $\mu_{1}, \mu_{2} \subseteq U$ tal que $\nu_{1}=\mu_{1} \mu_{2}$. Portanto, $m=\left(\mu_{1} \mu_{2}\right) \nu_{2} \subseteq \bar{U}^{2} V$.

Proposição 2.4 Para todo P-subespaço das álgebras de Bernstein que satisfazem (2.18) tem-se $U(U p) \subseteq p$.

Demonstraçìo: É suficiente provar a proposição para um P-monômio $m$. Usaremos indução no grau de $m$. Se $m=U$ ou $m=V$ entào

$$
\begin{aligned}
& U(U U)=U^{3} \subseteq U \\
& U(U V) \subseteq U^{2} \subseteq V
\end{aligned}
$$

Suponhamos que a proposiçào é verdadeira para todo $\mathrm{P}$-monômio de grau menor ou igual a um inteiro positivo $k$. Seja $m$ um P-monômio com $\partial m=k+1$. Existem duas possibilidades: $m \subseteq U$ ou $m \subseteq V$. Observemos que $\partial m \geq 2$. Assim, pelo Lema 2.1, se $m \subseteq U$ entào $m=V^{2}$ ou $m=\mu \nu$, onde $\mu \subseteq U$ e $\nu \subseteq V$ sào P-monômios com $\partial \mu, \partial \nu \leq k$. Agora, se $m \subseteq V$ entào, pela Proposição 1.1, existe um P-monômio $\mu^{\prime} \subseteq U$ tal que $m=U \mu^{\prime}$ e $\partial \mu^{\prime} \leq k$. Segue de (6) que

$$
U\left(U V^{2}\right)=0
$$

Usando $(b)$ e $(c)$ da Proposiçào 0.1 obtemos

$$
U(U(\mu \nu))=L(\mu(U \nu)) \subseteq \mu((U \nu) U)+(U \nu)(U \mu)
$$

Segue da parte $(a)$ do Corolário 2.2 e da hipótese de indução que

$$
U(U(\mu \nu)) \subseteq \mu(U(U \nu))+(U(U \mu)) \nu \subseteq \mu \nu
$$


Novamente, pela hipótese de indução.

$$
U\left(U\left(U \mu^{\prime}\right)\right) \subseteq U \mu^{\prime}
$$

Portanto, para qualquer um dos casos, temos $L(U m) \subseteq m$.

O próximo corolário no diz que os $\mathrm{P}$-subespaços das álgebras de Bernstein que satisfazem (2.18) absorvem produto por $U^{2}$.

Corolário 2.3 Em toda álgebra de Bernstein que satisfaz (2.18) tem-se $U^{2} p \subseteq p$. para qualquer P-subespaço $p$.

Demonstração: Escrevendo $p=g \oplus h \operatorname{com} g \subseteq L^{\circ} . h \subseteq V$ e usando a parte $(a)$ da Proposiçào 0.1 temos

$$
U^{2} p=U^{2} g+U^{2} h \subseteq U(U g)+U^{2} V
$$

Resulta daí e da Proposição 2.4 que

$$
U^{2} p \subseteq U(U g) \subseteq g \subseteq p
$$

Como conseqüência do corolário anterior temos a seguinte propriedade do automorfismo $\psi$.

Lema 2.2 . Vas álgebras de Bernstein que satisfazem (2.18) tem-se $\psi(g)=g$, para todo $P$-subespaço $g \subseteq U$.

Demonstraçio: C'omo భ'é um automorfismo de espaço vetorial, basta mostrar que $\psi(g) \subseteq g$. Seja $x \in g$. Visto que $u_{0}^{2} \in U^{2}$, usando o Corolário 2.3 . temos $\psi(x)=x-2 u_{0}^{2} x \in g+U^{2} g=g$.

Sejam $A=L \epsilon € U_{e} \boxplus V_{e}$ uma álgebra de Bernstein e $u_{0} \in U_{e}$. A transformação de Peirct associada a $\epsilon$ e a $u_{0}$ é a função linear $\varphi: A \rightarrow A$ definicla por $\varphi(\alpha e+u+v)=\alpha f+\sigma(u)+\tau(v)$. onde $f=\epsilon+u_{0}+u_{0}^{2}$. Assim, as transformaçòes de Peirce são automorfismos de espaço vetorial. Ver $[2]$ para maiores informaçòes sobre transformaçòes de Peirce em álgebras de Bernstein.

Provaremos agora o principal resultado desta seção.

Teorema 2.1 Todo P-subespaço de uma álgtbra de Bernstein que satisfaz (2.1S) tem dimension incariante. 
Demonstraçào: Sejam $A=K e \oplus U_{e} \doteq V_{e}=K f \oplus U_{f} \oplus V_{f}$ duas decomposiçòes de Peirce de A. onde $f=e+u_{0}+u_{0}^{2} \operatorname{com} u_{0} \in U_{\epsilon}$. Seja $\varphi$ a transformação de Peirce associada a $e$ e a $u_{0}$. Mostraremos que

$$
p\left(p_{e}\right)=p_{f}
$$

Inicialmente provaremos que (2.21) vale para um P-monômio $m$, fazendo indução no grau $k$ de $m$. Se $k=1$ então $m=U$ ou $m=V$. Temos que $\varphi\left(U_{e}\right)=\sigma\left(U_{\epsilon}\right)=U_{f}$ e, analogamente, $\varphi\left(V_{\epsilon}\right)=\tau\left(V_{e}\right)=V_{f}$. Suponhamos agora que (2.21) vale para todo P-monômio de grau menor ou igual a $k$. Seja $m$ um P-monômio de $A$ com $\partial m=k+1$. Portanto. pela Observação 1.2 e pelo Lema 2.1 , temos três possibilidades: $m=\mu \nu, m=V^{2}$ ou $m=\mu_{1} \mu_{2}$, onde $\mu, \mu_{1}$, $\mu_{2} \subseteq U$ e $\nu \subseteq V$ sào P-monômios de grau menor ou igual a $k$. Se $m=\mu \nu$ então segue da hipótese de induçào que

$$
m_{f}=\mu_{f} \nu_{f}=\varphi\left(\mu_{e}\right) \varphi\left(\nu_{e}\right)=\sigma\left(\mu_{e}\right) \tau\left(\nu_{e}\right)
$$

Portanto, pelo Corolário $\left.2.2, m_{f}=\sigma\left(\psi_{(} \mu_{\epsilon}\right) \nu_{e}\right)$ e, usando o Lema 2.2 , obtemos

$$
m_{f}=\sigma\left(\mu_{e} \nu_{e}\right)=\sigma\left(m_{e}\right)=\varphi\left(m_{e}\right)
$$

Os outros casos sào feitos de maneira análoga. Agora. seja $p$ um P-subespaço de A. Logo, existem P-monômios $m_{1}, \ldots, m_{k}$ tais que $p=\sum_{i=1}^{k} m_{i}$. Assim, escrevendo $m_{i}\left(U_{e}, V_{e}\right)=m_{i_{e}}$ e $m_{i}\left(U_{f}, V_{f}\right)=m_{i_{f}}$, temos que

$$
\vartheta\left(p_{e}\right)=\sum_{i=1}^{k} \varphi\left(m_{i_{e}}\right)=\sum_{i=1}^{k} m_{i_{f}}=p_{f}
$$

Como $\rho$ é um isomorfismo, $\operatorname{dim} p_{e}=\operatorname{dim} p_{f}$. Portanto. $p$ tem dimensão invariante.

Com o objetivo de obter um resultado mais geral enfraquecendo a condição (2.18), podemos fazer as seguintes perguntas:

1ㅡ) Se retirarmos uma das hipóteses $U^{2} V=0$ ou $(u v) v=0$ da condiçào $(2.18)$, o Teorema 2.1 ainda vale?

2a) Substituindo-se a hipótese $U^{2} V=0$ por uma mais fraca, por exemplo (ver figura 1.1 ):

$$
\begin{aligned}
V(U(U V)) & =0 \\
\left(U^{2}\right)^{2} & =0 \\
V\left(U\left(I^{\prime} V\right)\right)+\left(L^{2}\right)^{2} & =0
\end{aligned}
$$


o resultado do Teorema 2.1 ainda é verdadeiro?

Os Exemplos 2.2 e 2.4 mostram que a resposta para cacla uma destas perguntas é negativa. Vimos que as álgebras destes exemplos possuem P-subespaços que não têm dimensão invariante. Entretanto, no exemplo 2.2 temos

$$
\begin{aligned}
U_{e}^{2} V_{e} & \neq 0 \\
(u v) v \in\left(U_{e} V_{e}\right) V_{e} & =0 \\
V_{e}\left(U_{e}\left(U_{e} V_{e}\right)\right) & =0 \\
\left(U_{e}^{2}\right)^{2} & =0
\end{aligned}
$$

e, no Exemplo 2.4 temos

$$
\begin{aligned}
U_{\epsilon}^{2} V_{e} & =0 \\
\left(u_{1}\left(v_{1}+v_{3}\right)\right)\left(v_{1}+v_{3}\right) & =-\frac{1}{2} u_{5}
\end{aligned}
$$

Neste sentido. a condição (2.18) é a melhor estimativa para o Teorema 2.1.

Observação 2.2 Muitas vezes, de agora em diante, para simplificar a notação, escreveremos $\sigma\left(g_{e}\right)=g_{f} \in \tau\left(h_{e}\right)=h_{f}$ no lugar de $\sigma_{u}\left(g_{e}\right)=g_{f_{u}} \in \tau_{u}\left(h_{e}\right)=h_{f_{u}}$, respectivamente. Porém deve ficar claro que as funções $\sigma$ e $\tau$ dependem do par de idempotentes e e $f$.

O resultado seguinte é um corolário da demonstraçào do teorema anterior.

Corolário 2.4 Seja A uma álgebra de Bernstein que satisfaz (2.18). Se $g \subseteq U \in h \subseteq 1$. são P-subespaços de A entĩo

$$
\begin{aligned}
& \sigma\left(g_{e}\right)=g_{f} \\
& \tau\left(h_{\epsilon}\right)=h_{f}
\end{aligned}
$$

para quaisquer $\epsilon, f \in I p(A)$.

Agora, depois de estudarmos a invariância da dimensào de um P-subespaço, passamos a estudar a invariância do próprio $\mathrm{P}$-subespaço. O Teorema 2.2 a seguir nos fornece um critério para que um P-subespaço de uma álgebra de Bernstein que satisfaz (2.18) seja invariante.

Teorema 2.2 Seja p um P-subespaço de uma álgebra de Bernstein que satisfaz (2.18). Entào $p$ é incariante se. $\epsilon$ somente se. $L^{\dagger} p \subseteq p$. 
Demonstraçào: Seja $A=K \epsilon \oplus U_{e} \oplus V_{e}$ uma decomposição de Peirce de $A$ e seja $p=g \doteqdot h$ um P-subespaço de $A$ com $g \subseteq U$ e $h \subseteq V$. Para qualquer $u \in U_{e}$, seja $f=\epsilon+u+u^{2}$. Usando o Corolário 2.4, obtemos $p_{f}=g_{f} \uplus h_{f}=\left\{\sigma(x)+\tau(w) ; x \in g_{e}, w \in h_{e}\right\}$. Portanto.

$$
p_{f}=\left\{(x-2 u w)+(w+2 u x) ; x \in g_{e} w \in h_{e}\right\}
$$

Suponhamos que $p$ é invariante. Logo, $p_{f}=p_{e}$ para quaisquer idempotentes $e$ e $f$. Em particular, $p_{f} \subseteq p_{e}$. Portanto, segue de (2.22) que dados $x \in g_{e}$ e $w \in h_{e}$, existem $x^{\prime} \in g_{e}$ e $w^{\prime} \in h_{e}$ tais que $x-2 u w=x^{\prime}$ e $w+2 u x=w^{\prime}$. Assim,

$$
\begin{aligned}
u w & =\frac{1}{2}\left(x-x^{\prime}\right) \\
u x & =\frac{1}{2}\left(w^{\prime}-w\right)
\end{aligned}
$$

Logo, $U_{e} g_{e} \subseteq h_{e}$ e $U_{\epsilon} h_{e} \subseteq g_{e}$. De onde segue que $U_{\epsilon} p_{e} \subseteq p_{e}$.

Reciprocamente, suponhamos que $U p \subseteq p$. Podemos reescrever (2.22) como segue

$$
p_{f}=\left\{x+w+2 u(x-w) ; x \in g_{e}, w \in h_{e}\right\}
$$

de onde segue que

$$
p_{f} \subseteq p_{e}+U_{e} p_{e}
$$

Agora, usando a hipótese, concluímos que $p_{f} \subseteq p_{e}$. Como esta inclusão vale para qualquer par de idempotentes, segue-se que $p_{e}=p_{f}$. Assim. $p$ é invariante.

Daremos agora a descriçào do conjunto dos P-subespaços invariantes da uma álgebra de Bernstein que satisfaz (2.18). Lembramos que $\mathcal{P}$, com a identificaçào que já mencionamos antes, denota o conjunto de todos os P-subespaços de $A$.

Corolário 2.5 O conjunto dos P-subespaços invariantes de uma álgebra de Bernstein que satisfaz (2.18) t dado por $\{p+U p ; p \in \mathcal{P}\}$.

Demonstraçìo: Se $p$ é invariante entào, pelo Teorema 2.2, temos que $p=p+U p$. Por outro lado, se $p$ é um P-subespaço qualquer então. usando a Proposição 2.4, temos

$$
U(p+U p)=U p+U(U p) \subseteq U p+p
$$

Portanto. $p+l^{\prime} p$ é invariante.

Também poderíamos deduzir que $p+U p$ é invariante para qualquer P-subespaço $p$ de $A$ a partir da proposição seguinte. 
Proposição 2.5 Para todo P-subespaço p de uma álgebra de Bernstein que satisfaz (2.18) tem-se

$$
p+L p=\sum_{f \in \operatorname{Ip}(A)} p_{f}
$$

Demonstração: Seja $A=K \epsilon \exists U_{\epsilon}=V_{\epsilon}$ uma decomposição de Peirce de $A$. Escrevamos $p=g \oplus h \operatorname{com} g \subseteq U$ e $h \subseteq V$. Para quaisquer $u \in U_{e}, x \in g_{e}$ e $w \in h_{e}$, segue do Corolário 2.4 que

$$
\begin{aligned}
u x & =-\frac{1}{2}\left(x-\sigma_{u}(x)\right) \in g_{e}+g_{f_{u}} \\
u w & =\frac{1}{2}\left(w-\tau_{u}(w)\right) \in h_{e}+h_{f_{u}}
\end{aligned}
$$

Portanto, $U_{e} g_{e} \subseteq \sum_{f \in \operatorname{Ip}(A)} g_{f}$ e $U_{e} h_{e} \subseteq \sum_{f \in \operatorname{Ip}(A)} h_{f}$. Daí segue que

$$
U_{e} p_{e}=U_{e} g_{e}+U_{e} h_{e} \subseteq \sum_{f \in \operatorname{Ip}(A)} g_{f}+\sum_{f \in \operatorname{Ip}(A)} h_{f}=\sum_{f \in \operatorname{Ip}(A)}\left(g_{f}+h_{f}\right)=\sum_{f \in \operatorname{Ip}(A)} p_{f}
$$

Logo, $p_{e}+U_{e} p_{e} \subseteq \sum_{f \in \operatorname{Ip}(A)} p_{f}$. Por outro lado, segue de (2.23) que $\sum_{f \in \operatorname{Ip}(A)} p_{f} \subseteq p+U p$.

Dado $p$ um P-subespaço qualquer de uma álgebra de Bernstein $A$, o menor subespaço vetorial de $A$ que contém a família $\left\{p_{f}\right\}_{f \in \operatorname{Ip}(A)}$ é dado por $\sum_{f \in \operatorname{Ip}(A)} p_{f}$. A proposição anterior nos diz que este subespaço, no caso em que A satisfaz (2.18), é um P-subespaço e tais subespaços formam o conjunto de todos os P-subespaços invariantes de $\mathrm{A}$.

\subsection{Invariância em álgebras de Bernstein-Jordan}

Como já tínhamos observado antes, as álgebras de Bernstein-Jordan satisfazem (2.18). Portanto os resultados da seçào anterior sào válidos também para esta subclasse. Assim temos o seguinte corolário do Teorema 2.1.

Corolário 2.6 Em uma álgebra de Bernstein-Jordan todos os P-subespaços têm dimensão invariante.

Também temos que se $e$ e $f=\epsilon+u+u^{2}$ sào dois idempotentes de uma álgebra de Bernstein-Jordan com $u \in U_{\epsilon}$, então a transformação de Peirce $\rho$ associada a $e$ e a $u$ satisfaz

$$
\hat{r}\left(p_{\epsilon}\right)=p_{j}
$$


para todo P-subespaço $p$. Em particular se $g \subseteq U$ e $h \subseteq V$ são P-subespaços então

$$
\begin{aligned}
& \sigma\left(g_{e}\right)=g_{f} \\
& \tau\left(h_{e}\right)=h_{f}
\end{aligned}
$$

Como toda álgebra de Bernstein normal é de Bernstein-Jordan, temos que todo Psubespaço de uma álgebra de Bernstein normal tem dimensão invariante. Este fato nào é verdadeiro para álgebras de Bernstein excepcionais. Com efeito, seja $A=K e \oplus\langle u\rangle \oplus\langle v\rangle$ com tábua de multiplicaçào dada por $e^{2}=\epsilon . \epsilon u=\frac{1}{2} u, u v=u, v^{2}=4 u$ e os outros produtos iguais a zero. Para os idempotentes $e$ e $f=\epsilon+u$ temos

$$
\begin{aligned}
& V_{\epsilon}^{2}=\langle u\rangle \\
& V_{f}^{2}=0
\end{aligned}
$$

Nas álgebras de Bernstein-Jordan alguns resultados da seçào anterior podem ser melhorados. Isso se deve ao fato da condição $V^{2}=0$ ser mais forte que $U^{2} V=0$. Uma conseqüência disto é a seguinte observação:

Observação 2.3 Se $m$ é um P-monômio não nulo de uma álgebra de Bernstein-Jordan com $\partial m \geq 2$. entìo existem duas possibilidades: $m=\mu \nu$ ou $m=\mu_{1} \mu_{2}$, onde $\mu, \mu_{1}, \mu_{2} \subseteq U$ e $\nu \subseteq V$ são P-monômios de grau menor que $\partial \mathrm{m}$.

Segue desta observação a seguinte proposição cujo resultado é mais forte que o do Corolário 2.3 .

Proposição 2.6 Em toda álgebra de Bernstein-Jordan tem-se $V p \subseteq p$, para qualquer Psubespaço p.

Demonstração: Inicialmente faremos a demonstração para um P-monômio $m$. Se $m \subseteq V$ entào $V m \subseteq V^{2}=0$. Assim, basta provar para P-monômios contidos em $U$. Faremos indução no grau do P-monômio. Para $m=U$ temos $V U \subseteq U$. Suponhamos que o resultado vale para $\mathrm{P}$-monômios de grau menor ou igual a $k$. Seja $m$ com $\partial m=k+1$. Logo. pela Observação 2.3, $m=\mu \nu$. onde $\mu \subseteq U$ e $\nu \subseteq V$ sào P-monômios com $\partial \mu, \partial \nu<k$. Usando a Proposição 0.4 e a hipótese de indução obtemos

$$
V m=V(\mu \nu)=\nu(\mu V) \subseteq \nu \mu=m
$$

Seja agora $p=\sum_{i=1}^{r} m_{i}$ um P-subespaço, onde $m_{i} \ldots m_{r}$ são P-monômios. Temos que

$$
V p=\sum_{i=1}^{r} V m_{i} \subseteq \sum_{i=1}^{r} m_{i}=p
$$


Com esta proposiçào, para álgebras de Bernstein-Jordan. além da questão da invariância de um P-subespaço, podemos também responder a quinta pergunta da página 14 .

Proposição 2.7 Seja $p$ um P-subespaço de uma álgebra de Bernstein-Jordan. As seguintes afirmaçòes são equivalentes:

(a) pé invariante;

(b) $U p \subseteq p$;

(c) pé ideal.

Demonstração: Segue do Teorema 2.2 que $(a)$ é equivalente a $(b)$. Mostremos que $(b)$ é equivalente a $(c)$. Visto que $A=K e \oplus \zeta \doteqdot V$. vemos que $p$ é ideal se, e somente se, $\epsilon p \subseteq p$, $U p \subseteq p$ e $V p \subseteq p$. Fazendo a decomposiçào $p=g \pm h$ com $g \subseteq U$ e $h \subseteq V$. temos $e p=g \subseteq p$ e. pela Proposição 2.6, $V p \subseteq p$. Assim, $p$ é ideal se, e somente se, $U p \subseteq p$.

Reunimos no corolário seguinte os resultados do Corolário 2.5 e da Proposição 2.5, restritos ao caso das álgebras de Bernstein-.Jordan.

Corolário 2.7 Seja A uma álgebra de Bernstein-Jordan. Então

(a) $p+U p=\sum_{f \in \operatorname{Ip}(A)} p_{f}$, para todo P-subespaço $p$ de A:

(b) O conjunto dos P-subespaços invariantes de A é dado por $\{p+U p ; p \in \mathcal{P}\}$.

\subsection{Invariância em álgebras de Bernstein}

Nosso principal objetivo agora é encontrar condiçòes necessárias e suficientes para que um P-subespaço de uma álgebra de Bernstein seja invariante. Infelizmente para a classe inteira das álgebras de Bernstein nào foi possivel achar uma condiçào de fácil verificaçào.

O próximo resultado segue do Corolário 2.6.

Corolário 2.8 Todo P-subespaço de uma álgebra de Bernstein contido em I' tem dimensào invariante.

Demonstraçào: Seja $A=l i \epsilon \unlhd U \triangleleft V$ a decomposiçào de Peirce de uma álgebra de Bernstein A relativa ao idempotente $\epsilon$. Consideremos a decomposiçào de Peirce $\bar{A}=K^{\prime} \bar{\epsilon} \oplus \bar{U} \oplus \bar{V}$ 
da álgebra de Bernstein $\bar{A}=A / L$ associada ao idempotente $\bar{\epsilon}=e+L$. Para todo Psubespaço $h(U, V)$ de $A$ contido em $V$ temos $h(\bar{U}, \bar{V}) \subseteq \bar{V}$ e $h(\bar{U}, \bar{V})=(h(U, V) \oplus L) / L$. Portanto, $\operatorname{dim} h(U, V)=\operatorname{dim} h(\bar{U}, \bar{V})$. Agora, $h(\bar{U}, \bar{V})$ tem dimensào invariante, pois $\bar{A}$ é de Bernstein-Jordan. Logo, $h(U, V)$ também tem dimensão invariante.

Os P-subespaços das família a seguir estão contidos em V. logo, pelo corolário anterior, têm dimensào invariante ( $k$ e $r$ são inteiros positivos).

$$
\begin{aligned}
& U^{2 k} \\
& U^{(2 k)} V \\
& (U V)^{2 k} \\
& U^{(2 k+1)} U^{(2 r+1)} \\
& U^{(2 k+1)}\left(U V^{(r)}\right) \\
& \left(U V^{(k)}\right)\left(U V^{(r)}\right)
\end{aligned}
$$

O próximo corolário segue imediatamente do Corolário 2.8. Com ele podemos produzir P-subespaços contidos em $U$ que têm dimensão invariante.

Corolário 2.9 Seja $p=g \pm h$ um P-subespaço de uma álgebra de Bernstein com $g \subseteq U \in$ $h \subseteq V$. Entào p tem dimensão invariante se, e somente se, g tem dimensão invariante.

É claro que se $p$ é invariante então $p$ tem dimensão invariante. Assim, usando os métodos de fabricar P-subespaços invariantes dados na Seçào 2.1, podemos produzir outros P-subespaços de dimensão invariante.

Estudamos na Subseção 2.1.2 os subespaços invariantes $S_{k+1, k}, S_{k, k}$ e $S_{k, k+}$. Agora. usando os Corolários 2.8 e 2.9 , concluímos que $S_{k, U}$ e $S_{k, 1}$ têm dimensào invariante. A partir de 2.15 e 2.16 podemos construir o seguinte diagrama. com as retas tendo o mesmo significado dos diagramas anteriores. 


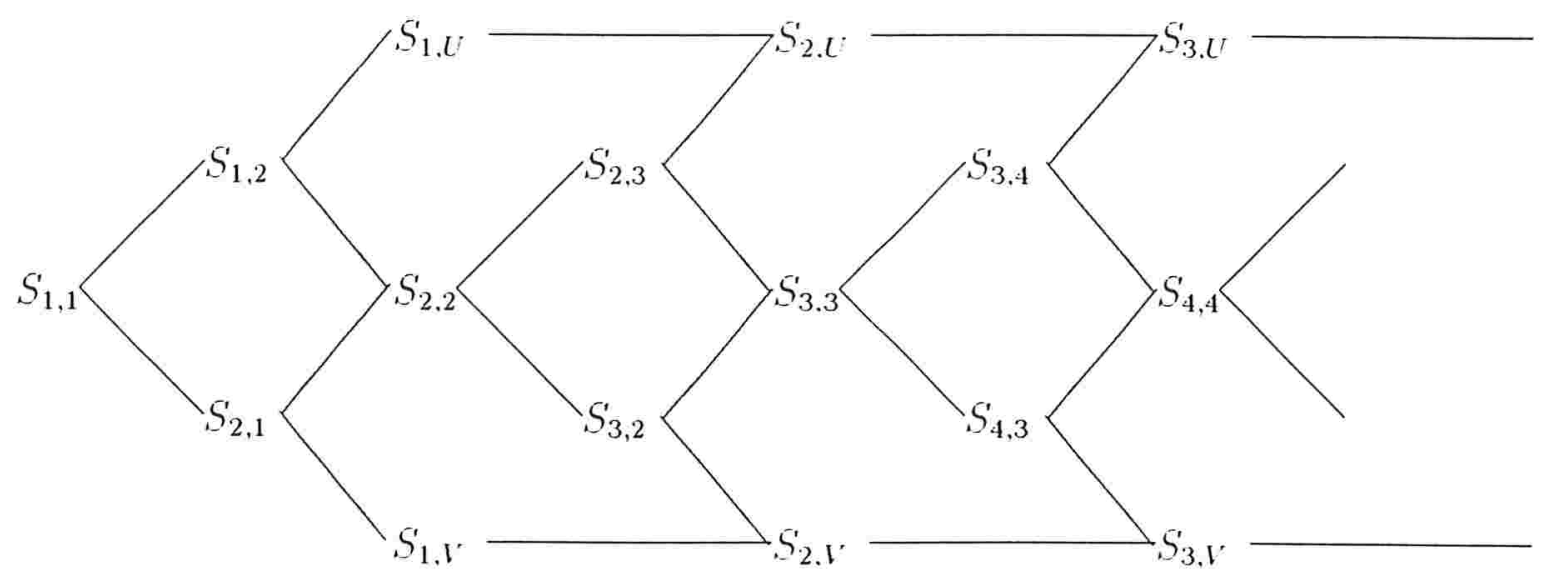

Figura 2.1: P-subespaços $S_{k+1, k}, S_{k, k}, S_{k, k+1}, S_{k, U}$ e $S_{k, V}$.

A este diagrama está associado um diagrama numérico, que se obtém substituindo cada subespaço pela sua respectiva dimensào. Este novo diagrama é um invariante da álgebra.

A próxima proposição nos diz que se um $\mathrm{P}$-subespaço está contido em $V$ entào a mudança de idempotente é feita de maneira simples.

Proposição 2.8 Se hé um P-subespaço de uma álgebra de Bernstein contido em V. então $\tau\left(h_{e}\right)=h_{f}$, para quaisquer idempotentes $e \in f$.

Demonstração: Seja $A$ uma álgebra de Bernstein e sejam $e, f \in \operatorname{Ip}(A) \operatorname{com} f=\epsilon+u+u^{2}$, onde $u \in U_{e}$. Consideremos a álgebra quociente $\bar{A}=A / L$. Usaremos a notação $\bar{x}=x+L$, para qualquer $x \in A$. Sejam as decomposiçòes de Peirce $\bar{A}=\Lambda \bar{e} \oplus \bar{U} \bar{\epsilon} \oplus \bar{V}_{\bar{\epsilon}}=K \bar{f} \overline{\bar{U}} \bar{f} \Phi \bar{V}_{\bar{f}}$ de $\bar{A}$ associadas aos idempotentes $\bar{e}$ e $\bar{f}$. Seja $\pi: A \rightarrow \bar{A}$ a projeçào $\pi(x)=\bar{x}$. Segue da definiçào de subespaços polinomiais que

$$
p\left(\bar{U}_{\bar{\epsilon}}, \bar{V}_{\bar{\epsilon}}\right)=\pi\left(p\left(U_{\epsilon}^{\prime}, V_{\epsilon}\right)\right)
$$

para qualquer P-subespaço $p$ de $A$. Temos que $\bar{f}=\overline{\epsilon+u+u^{2}}=\bar{\epsilon}+\bar{u}+\bar{u}^{2}$. Sejam $\tau: V_{e} \rightarrow V_{f}$ e $\bar{\tau}: \bar{V}_{\bar{\epsilon}} \rightarrow \bar{V}_{\bar{f}}$ os isomorfismos de espaços vetoriais definidos em (21). isto é, para todo $v \in V_{e}, \tau(v)=v-2 u v-2 u^{2} v$ e $\bar{\tau}(\bar{v})=\bar{v}-2 \overline{u v}$, visto que $u^{2} v \in L$. Portanto, $\bar{\tau}(\bar{v})=\overline{\tau(v)}$. Temos que $\left.\bar{\tau} \circ \pi\right|_{v_{s}}=\left.\pi\right|_{v_{f}} \circ \tau$. De fato. $\bar{\tau}(\pi(v))=\bar{\tau}(\bar{v})=\overline{\tau(v)}=\pi(\tau(v))$. Assim,

$$
\bar{\tau}\left(h\left(\bar{U}_{\bar{e}}, \bar{V}_{\bar{\epsilon}}\right)\right)=\bar{\tau}\left(\pi\left(h\left(V_{\epsilon}, V_{e}\right)\right)\right)=\pi\left(\tau\left(h\left(L_{\epsilon}, V_{\epsilon}\right)\right)\right)=\left(\tau\left(h\left(V_{\epsilon}, V_{\epsilon}\right)\right) \notin L\right) / L
$$

Por outro lado, sendo A Bernstein-Jordan. segue de (2.25) que

$$
\bar{\tau}\left(h\left(\bar{U}_{\bar{\epsilon}}, \bar{V}_{\bar{\epsilon}}\right)\right)=h\left(\bar{U}_{\bar{f}}, \bar{V}_{\bar{f}}\right)=\left(h\left(U_{f}, V_{f}\right) \pm L\right) / L
$$


Concluímos que

$$
\left(h\left(U_{f}, V_{f}\right) \oplus L\right) / L=\left(\tau\left(h\left(L_{e}^{r}, V_{\epsilon}\right)\right) \oplus L\right) / L
$$

Portanto, se $w \in h\left(U_{f}, V_{f}\right)$ entào $w+L \in\left(h\left(U_{f}, V_{f}\right) \oplus L\right) / L$. Por (2.26), existe $w^{\prime} \in$ $h\left(U_{e}, V_{e}\right)$ tal que $w+L=\tau\left(w^{\prime}\right)+L$, ou seja, $w-\tau\left(w^{\prime}\right) \in L$. Portanto, $w-\tau\left(w^{\prime}\right) \in V_{f} \cap L=0$. Logo, $w=\tau\left(w^{\prime}\right)$. Assim. $h\left(U_{f} . V_{f}\right) \subseteq \tau\left(h\left(U_{e}, V_{e}^{\prime}\right)\right)$. Mas, pelo Corolário 2.8, $h$ tem dimensão invariante. Portanto, $h\left(U_{f} . V_{f}\right)=\tau\left(h\left(U_{\epsilon}, V_{\epsilon}\right)\right)$.

A proposição seguinte e o seu corolário fornecem duas condiçòes necessárias para a invariância de um P-subespaço nas álgebras de Bernstein.

Proposição 2.9 Seja $p=g \Xi h$ um P-subespaço de uma álgebra de Bernstein com $g \subseteq U$ e $h \subseteq V$. Se $p$ é invariante então $U g \subseteq h$.

Demonstração: Se $p(U . V)$ é um P-subespaço invariante de uma álgebra de Bernstein A então $p(\bar{U}, \bar{V})$ é um P-subespaço invariante de $\bar{A}=A / L$. Como $\bar{A}$ é Bernstein-Jordan, segue da Proposição $2 . \bar{\imath}$ que $\overline{{ }^{I}} p(\bar{U}, \bar{V}) \subseteq p(\bar{U}, \bar{V})$. Logo, $\bar{U} g(\bar{U}, \bar{V}) \subseteq h(\bar{U}, \bar{V})$. De onde segue que $U g(U, V) \subseteq h(U, V)$. De fato, se $w \in U g(U, V)$, entào $w+L \in \bar{U} g(\bar{U}, \bar{V})$. Logo, $w+L \in h(\bar{U}, \bar{V})$. Portanto, existe $z \in h(U, V)$ tal que $w+L=z+L$, ou seja, $x-z \in L$. Logo, $w=z$.

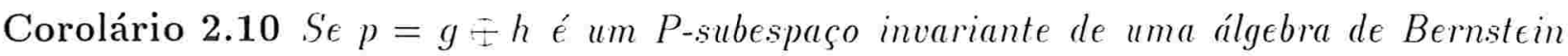
com $g \subseteq U \in h \subseteq V$, então $\sigma\left(g_{e}\right)=g_{f}$, para quaisquer $e, f \in I p(A)$.

Demonstraçào: Sejam $\epsilon . f \in \operatorname{Ip}(A) \operatorname{com} f=e+u+u^{2}$, onde $u \in L_{e}^{*}$. Como $p=g \Phi h$ é invariante. $g$ tem dimensào invariante. Assim, basta mostrar que $\sigma\left(g_{\varepsilon}\right) \subseteq g_{f}$. Seja $x \in g_{\varepsilon}$. Logo, $\sigma(x)=x+2 u x \in g_{e} \in l_{e} g_{e}$. Pela Proposiçào 2.9, $g_{e} \oplus U_{e} g_{e} \subseteq g_{e} \boxplus h_{e}=p_{e}$. Sendo $p$ invariante, temos $p_{e}=p_{f}=g_{f} \pm h_{f}$. Portanto, $\sigma(x) \in g_{f} \boxplus h_{f}$. De onde segue que $\sigma(x) \in g_{f}$.

Agora estamos prontos para dar condiçòes necessárias e suficientes para que um Psubespaço seja invariante numa álgebra de Bernstein arbitrária.

Teorema 2.3 Seja $p=g \pm h$ um P-subespaço de uma álgebra de Bernstein, onde $g \subseteq l t$ $h \subseteq V$. As seguintes afirmaçòes são equivalentes: 
(a) pé incrariante

(b) $\left\{\begin{array}{l}U^{\prime} p \subseteq p \\ U^{2} h \subseteq g \\ \sigma\left(g_{e}\right)=g_{f} \text { para quaisquer } e, f \in I p(A)\end{array}\right.$

(c) $\left\{\begin{array}{l}U h \subseteq g \\ U^{2} h \subseteq g \\ g_{f} \subseteq p_{e} \text { para quaisquer } e, f \in I p(.-1)\end{array}\right.$

Demonstraçào: Mostremos que (a) implica (b). Se $p$ é invariante então $p_{f_{u}} \subseteq p_{e}$. para quaisquer $\epsilon \in \operatorname{Ip}(A)$ e $u \in U_{\epsilon}$. Além disso. pelo Corolário $2.10, \sigma\left(g_{e}\right)=g_{f}$. Assim. usando a Proposiçào 2.8, temos

$$
\begin{aligned}
p_{f_{u}} & =\sigma_{u}\left(g_{e}\right) \oplus \tau_{u}\left(h_{e}\right) \\
& =\left\{\sigma_{u}(x)+\tau_{u}(w) ; x \in g_{e} \mathrm{e} w \in h_{e}\right\} \\
& =\left\{\left(x-2 u w-2 u^{2} w\right)+(w+2 u x) ; x \in g_{e} \mathrm{e} w \in h_{e}\right\}
\end{aligned}
$$

Portanto,

$$
\left\{\left(x-2 u w-2 u^{2} w\right)+(w+2 u x) ; x \in g_{e} \text { e } w \in h_{e}\right\} \subseteq\left\{x^{\prime}+w^{\prime} ; x^{\prime} \in g_{e} \text { e } w^{\prime} \in h_{e}\right\}
$$

De onde segue que

$$
\left\{\begin{array}{l}
u x=\frac{1}{2}\left(u^{\prime}-u^{\prime}\right) \\
\left(u+u^{2}\right) u=\frac{1}{2}\left(x-x^{\prime}\right)
\end{array}\right.
$$

Como, ux é um gerador de ${ }^{I} g_{e}$ e, pela parte (b) do Lema $0.1,\left(u+u^{2}\right) w$ é um gerador de $U h+U^{2} h$, tem-se que

$$
\left\{\begin{array}{l}
l_{e}^{*} g_{e} \subseteq h_{e} \\
l_{\epsilon} h_{e} \subseteq g_{e} \\
l_{\epsilon}^{2} h_{e} \subseteq g_{e}
\end{array}\right.
$$

As duas primeiras inclusões implicam que $l_{\epsilon}^{*} p_{E} \subseteq p_{e}$.

Mostremos que (b) implica (c). Segue de $l p \subseteq p$ que $l h \subseteq g$ e $l g \subseteq h$. Dados $c$. $f \in \operatorname{Ip}(. A)$ temos

$$
g_{f}=\sigma\left(g_{e}\right) \subseteq g_{e}+l_{\epsilon} g_{e} \subseteq g_{e}+h_{e}=p_{e}
$$


Mostremos agora que (c) implica (a). Sejam $\epsilon, f \in \operatorname{Ip}(A)$. Usando a Proposição 2.8. temos

$$
p_{f}=g_{f}+\tau\left(h_{e}\right) \subseteq p_{e}+h_{e}+U_{e} h_{e}+U_{e}^{2} h_{e}=p_{e}
$$

Portanto, $p$ é invariante.

Segue de $U^{2} h \subseteq U^{2} V$, para todo P-subespaço $h \subseteq V$, e do Corolário 2.4 que nas álgebras de Bernstein que satisfazem (2.18) as condiçōes $U^{2} h \subseteq g$ e $\sigma\left(g_{e}\right)=g$, sào satisfeitas. Assim, o Teorema 2.3 é uma generalização do Teorema 2.2.

Vimos na Seçào 2.4 que um P-subespaço $p$ de uma álgebra de Bernstein-Jordan é invariante se, e somente se. $p$ é um ideal. Em uma álgebra de Bernstein arbitrária não há relação entre ser ideal e ser P-subespaço invariante. De fato. os ideais de uma álgebra de Bernstein que sào $\mathrm{P}$-subespaços nào são necessariamente invariantes. Consideremos, por exemplo, o ideal $I_{\epsilon}=\left[U_{\epsilon} V_{\epsilon}+V_{e}\left(U_{\epsilon}\left(U_{\epsilon} V_{\epsilon}\right)\right)\right] \oplus U_{\epsilon}\left(U_{e} V_{\epsilon}\right)$, e a álgebra do exemplo 2.3. Temos que $I_{\epsilon}$ nào é invariante. pois $U_{e} V_{e}=0$, logo, $I_{e}=0$. mas para o idempotente $f=e+u_{1}+u_{2}+v_{1}+v_{2}+2 v_{3}, U_{f} V_{f}=\left\langle u_{3}\right\rangle$, assim, $I_{f} \neq 0$. Também nem todo P-subespaço invariante é icleal, por exemplo. o P-subespaço $\tilde{V}=\left(U V+U^{2} V\right) \oplus V$ é invariante, mas na álgebra de Bernstein $A=K^{\prime} e \fallingdotseq\langle u\rangle \oplus\langle v\rangle$ com tábua de multiplicaçào dada por $e u=\frac{1}{2} u$, $v^{2}=u$ e os outros proclutos nulos, $\tilde{V}$ nào é icleal, pois $A \tilde{V}=\langle u\rangle \nsubseteq\langle v\rangle=\tilde{V}$ (ver também $[8])$.

Observemos que a hipótese $\sigma\left(g_{e}\right)=g_{f}$ não pode ser retirada da parte (b) do Teorema 2.3 , pois, caso contrário, todos os $\mathrm{P}$-subespaços que sào ideais de uma álgebra de Bernstein seriam invariantes.

Embora um P-subespaço invariante $p=g \nsubseteq h$, com $g \subseteq U$ e $h \subseteq V$. não seja necessarianmente um ideal de uma álgebra de Bernstein $A$, se $o(h) \geq 2$ entào $p$ é um ideal de $A^{2}$. conforme podemos ver a partir do próximo corolário.

Corolário 2.11 Se pé um P-subespaço invariante de uma álgebra de Bernstein A, entào $A^{2} p \subseteq p$.

Demonstraçaio: Escrevamos $p=g \oplus h \operatorname{com} g \subseteq U$ e $h \subseteq V$. Já sabemos, da parte (a) do Teorema 2.3 que $\left[p, L^{2} h \subseteq p\right.$. Esando a Proposiçào 0.1 . temos

$$
U^{2} g \subseteq U(U g) \subseteq U h \subseteq p
$$

Como $A=L \epsilon \div L^{*} \div L^{-2}$ e $t p=g$, segue-se que $A^{2} p \subseteq p$.

Corolário 2.12 Se p é um P-subespaço de uma álgebra de Bernstein nuclear então p é um ideal. 
O próximo resultado é uma generalizaçào da Proposição 0.2 . Faremos a prova deste caso mais geral embora nào haja uma diferença essencial entre as duas demonstraçòes.

Proposição 2.10 Para todo P-subespaço $g \subseteq U$ que satisfa $\sigma\left(g_{e}\right)=g_{f}$, para quaisquer $\epsilon$. $f \in I p(A)$, e para todo P-subespaço $h \subseteq V$, tem-se

(a) $\bigcap_{f \in \operatorname{Ip}(A)} g_{f}=g \cap \operatorname{ann} U$ :

(b) $\bigcap_{f \in \operatorname{Ip}(A)} h_{f}=h \cap \operatorname{ann}\left(U+U^{2}\right)$;

(c) $\sum_{f \in \operatorname{Ip}(A)} g_{f}=g \mp U g$;

(d) $\sum_{f \in \operatorname{Ip}(A)} h_{j}=\left(C^{h} h+U^{2} h\right) \oplus h$.

Demonstração:

(a) Seja $e \in \operatorname{Ip}(A)$ fixo. Se $x \in \bigcap_{f \in \operatorname{Ip}(A)} g_{f}$ então $x \in g_{e}$. Mostremos que $x \in \operatorname{ann} U_{\epsilon}$. De fato. temos que $x \in g_{f_{u}}$. para todo $u \in U_{e}$. Logo, existe $y \in g_{e}$ tal que $x=\sigma_{u}(y)=y+2 u y$. De onde segue que $x=y$ e $u y=0$. Portanto, $x \in \operatorname{ann} U_{\epsilon}$. Assim,

$$
\bigcap_{f \in \operatorname{Ip}(A)} g_{f} \subseteq g_{e} \cap \operatorname{ann} l_{e}^{\prime}
$$

Por outro lado, se $x \in g_{\epsilon} \cap \operatorname{nann} U_{e}$ então, para todo $u \in U_{e}$, tem-se $x=x+2 u x=\sigma_{u}(x) \in g_{I_{u}}$. Logo $x \in \bigcap_{f \in \operatorname{Ip}(A)} g_{f}$. Portanto,

$$
g_{e} \cap \operatorname{ann} L_{\epsilon}^{\prime} \subseteq \bigcap_{f \in \operatorname{Ip}(A)} g_{f}
$$

(b) Se $w \in \bigcap_{f \in \operatorname{Ip}(-A)} h_{f}$, entào. para todo $u \in U_{\epsilon}$, segue da Proposiçào 2.8 que $w \in h_{f_{u}}=\tau_{u}\left(h_{*}\right)$.

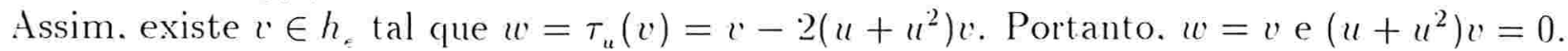
Usando o Lema 0.1. concluímos que $v \in \operatorname{ann}\left(U+U^{2}\right)$. Agora. se $w \in h_{e} \cap \operatorname{ann}\left(L_{\epsilon}^{\prime}+L_{\epsilon}^{-2}\right)$ então $w=w-2\left(u+u^{2}\right) w=\tau_{u}(w) \in h_{\rho_{u}}$. Logo. $w \in \bigcap_{f \in \operatorname{IP}(A)} h_{f}$.

(c) Para todo $u \in g_{e}$, temos $\sigma_{u}\left(g_{e}\right) \subseteq g_{e}+I_{\epsilon} g_{e}$. Portanto.

$$
\sum_{j \in \mathrm{Ip}(A)} g_{f}=\sum_{u \in U_{e}} g_{f_{u}}=\sum_{u \in L_{e}} \sigma_{u}\left(g_{e}\right) \subseteq g_{e}+U_{\epsilon} g_{e}
$$


Por outro lado. é claro que $g_{e} \subseteq \sum_{f \in \operatorname{Ip}(A)} g_{f}$ e se $x \in g_{e}$ entào $u x=\frac{1}{2}\left(\sigma_{u}(x)-x\right) \in g_{I_{u}}+g_{e}$. Portanto,

$$
U_{\epsilon} g_{e} \subseteq \sum_{f \in \mathrm{Ip}(A)} g_{f}
$$

(d) Segue de $h_{f_{u}}=\tau_{u}\left(h_{e}\right) \subseteq\left(U_{e} h+U_{e}^{2} h_{e}\right) \mp h_{e}$ que

$$
\sum_{f \in \operatorname{Ip}(A)} h_{f} \subseteq\left(U_{\epsilon} h_{e}+U_{e}^{2} h_{e}\right) \oplus h_{e}
$$

Observemos que $\left(u+u^{2}\right) w=\frac{1}{2}\left(w-\tau_{u}(w)\right) \in h_{e}+h_{f_{u}}$, para todo $w \in h_{e}$. Como $\left(u+u^{2}\right) w$ é um gerador de $\left(\zeta_{e}+L_{\epsilon}^{2}\right) h_{e}$, temos que

$$
h_{e} \subseteq\left(U_{\epsilon}+U_{\epsilon}^{2}\right) h_{e} \subseteq h_{e}+\sum_{u \in U_{e}} h_{f_{u}} \subseteq \sum_{f \in \operatorname{Ip}(A)} h_{f}
$$

Nas hipóteses da proposição anterior, temos

$$
\bigcap_{f \in \operatorname{Ip}(A)} g_{f}=g \cap \operatorname{ann} U=(g \cap U) \cap \operatorname{ann} U=g \cap(U \cap \operatorname{ann} U)=g \cap L
$$

Isto prova o seguinte corolário.

Corolário 2.13 Seja $p=g \boxplus h$ um P-subespaço de uma álgebra de Bernstein com $g \subseteq U$ $\epsilon h \subseteq V$. Se pé invariante entrio $\bigcap_{j \in \operatorname{Ip}(A)} g_{f}=g \cap L$.

O próximo resultado segue da parte (c) da Proposiçào 2.10.

Corolário 2.14 Seja $g \subseteq U$ um P-subespaço de uma álgebra de Bernstein. Entào $\sigma\left(g_{\epsilon}\right)=$ g para quaisquer $e, f \in I p(. t)$ se. $e$ somente se. $g \nsubseteq t g$ é incariante.

Demonstraçăo: Se $\sigma\left(g_{t}\right)=g_{f}$ para quaisquer $\epsilon, f \in \operatorname{Ip}(f)$. entào $g: l g=\sum_{f \in \operatorname{Ip}(A)} g_{f}$. Logo, $g \nsubseteq L g$ é invariante. Reciprocamente, se $g \leftarrow L^{\prime} g$ é invariante entào. pelo Teorema 2.3, $\sigma\left(g_{e}\right)=g_{f}$.

Corolário 2.15 Se $g \div h$ é um P-subespaço invariante de uma álgebra de Bernstein com $g \subseteq U \in h \subseteq V$. entào $g \subseteq l g$ também é invariante. 
Demonstraçào: Se $g \nsubseteq h$ é invariante entào $\sigma\left(g_{\varepsilon}\right)=g_{f}$. Pelo corolário anterior, $g \mp l g$ é invariante.

A parte (d) da Proposiçào 2.10 implica no seguinte corolário.

Corolário 2.16 Para todo P-subespaço $h \subseteq V$ de uma álgebra de Bernstein, tem-se que $\left(U h+U^{2} h\right) \Phi h$ é invariante.

$\mathrm{O}$ próximo corolário descreve o conjunto dos $\mathrm{P}$-subespaços invariantes de uma álgebra de Bernstein arbitrária.

Corolário 2.17 O conjunto dos P-subespaços incariantes de uma álgebra de Bernstein $\epsilon ́$ dado por

$$
\left\{p+\left[p+L^{-2} p ; p=g+h \in \mathcal{F}, h \subseteq V, g \subseteq U . \sigma\left(g_{e}\right)=h_{f}, e, f \in I p(A)\right\}\right.
$$

Demonstração: Seja $p=g \oplus h$ um P-subespaço de uma álgebra de Bernstein $A$ com $g \subseteq U^{*}$ e $h \subseteq V$. Temos que $p+\left[p+U^{2} p \subseteq p+A^{2} p\right.$. Assim. se $p$ é invariante então, pelo Corolário 2.11, $p=p+U p+L^{-2} p$ e $\sigma\left(g_{e}\right)=g_{f}$, para quaisquer $\epsilon, f \in \operatorname{Ip}(A)$. Por outro lado, se $\sigma\left(g_{e}\right)=g_{f}$ então $g \doteqdot L^{\prime} g$ é invariante. Logo, $U^{2} g \subseteq A^{2} g \cap U \subseteq g \mathrm{e}$, assim,

$$
p+U p+U^{2} p=g+U g+h+U h+U^{2} h
$$

Portanto, pelo Corolário 2.16. $p+U p+C^{-2} p$ é invariante.

Segue dos Corolários 2.3 e 2.4 que este último resultado é uma generalização do Corolário 2.5 .

\subsubsection{P-subespaços de grau $\leq 3$}

Reunimos aqui os todos os P-subespaços de uma álgebra de Bernstein que têm grau menor ou igual a 3. Eles estào separados em duas listas: a dos que têm dimensão invariante e a dos que nào têm dimensào invariante. Dentre os que têm dimensão invariante destacamos aqueles que são invariantes. O trabalho já foi quase todo feito nas seções anteriores.

Teorema 2.4 Se $A=K \epsilon € U \mp V$ é uma decomposiçio de Peirce de uma álgebra de Bernstein (A,w) entào

(1) os subespaços a seguir têm dimensào invariante e os marcados com * sào invariantes. 
Grupo I :

U

$U V+V^{2}$

$U^{3}+V^{3}+(U V) V+U^{2} V$

$U V+U^{2} V$

\section{Grupo II :}

V

$U(L V)$

Grupo III:

$\star I^{*} \oplus V$

$\star L^{-} \oplus L^{2}$ L $\oplus U(U V)$

* $\left(U V+V^{2}\right) \oplus V$

* $\left(U V+V^{2}\right) \oplus U^{2}$

* $\left(U V+V^{2}\right) \oplus U(U V)$ $\left(U^{3}+V^{3}+(U V) V+U^{2} V\right) \oplus V$

$\star\left(U^{3}+V^{3}+(U V) V+U^{2} V\right) \oplus U^{2}$

$\star\left(U^{3}+V^{3}+(U V) V+U^{2} V\right) \oplus U(L V)$

* $\left(L^{r} V+U^{2} V\right) \oplus V$

* $\left(U V+C^{-2} V\right) \oplus U^{2}$
$V^{2}+U^{3}+(U V) V+V^{3}$

$V^{2}+(U V) V+V^{3}$

$U^{2} V+(U V) V+V^{3}$

(2) os subespaģos a seguir nào tem dimensão invariante. 
Grupo IV:

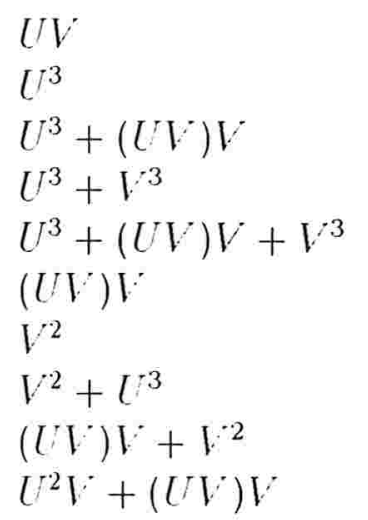

$V^{2}+U^{3}+(U V) V$

$(U V) V+V^{3}$

$U^{3}+U^{2} V+(U V) V$

$V^{3}$

$\mathrm{C}^{2} \mathrm{~V}$

$V^{2}+V^{3}$

$U^{2} V^{2}+V^{3}$

$V^{2}+U^{3}+I^{3}$

$I^{3}+I^{2} V+I^{3}$

$U^{3}+U^{2} V$

\section{Grupo V:}
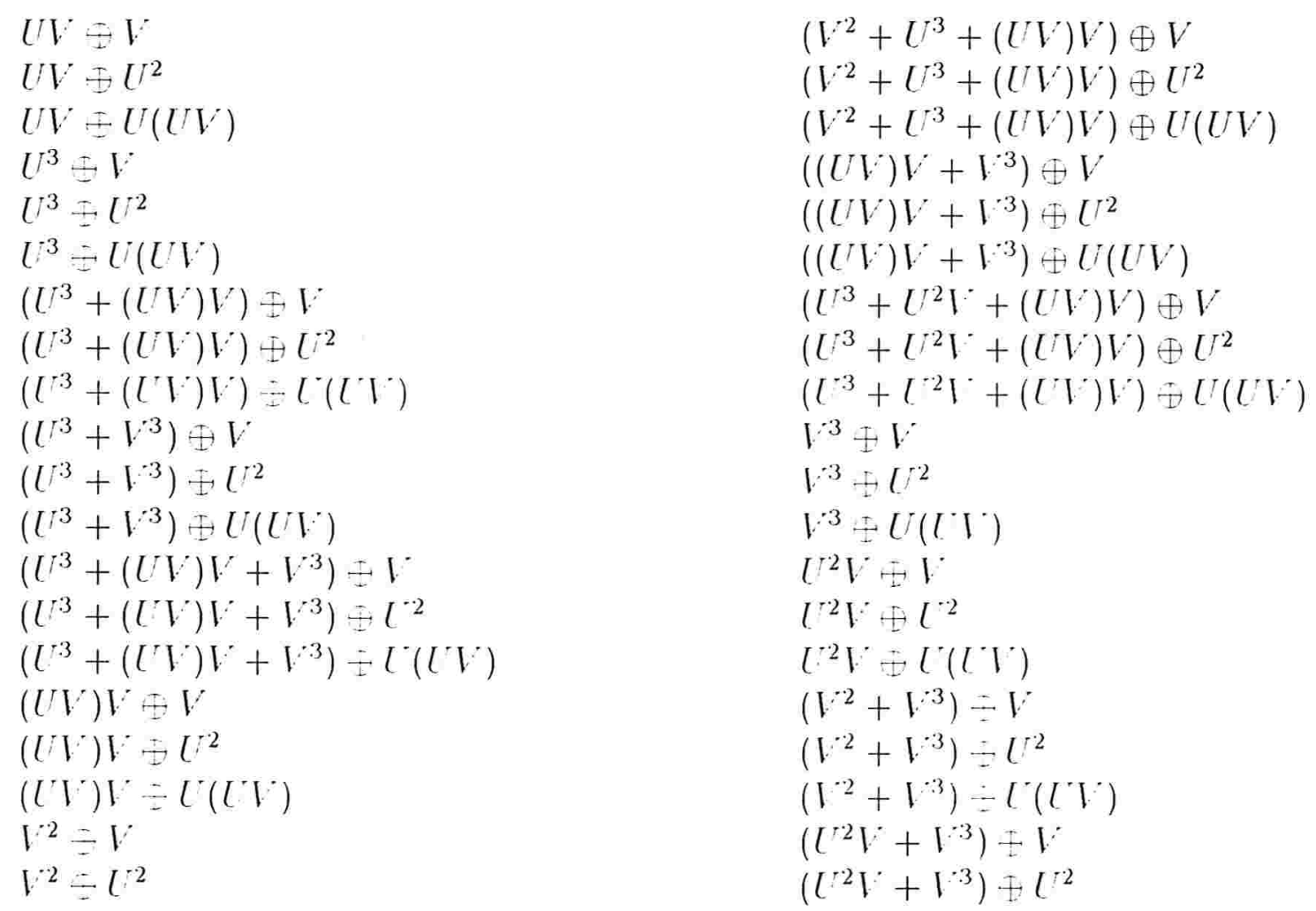


$$
\begin{array}{ll}
V^{2} \oplus U(U V) & \left(U^{2} V+V^{3}\right) \oplus U(U V) \\
\left(V^{2}+U^{-3}\right) \oplus V & \left(V^{2}+U^{3}+V^{3}\right) \oplus V \\
\left(V^{2}+U^{3}\right) \oplus U^{2} & \left(V^{2}+U^{3}+V^{3}\right) \oplus U^{2} \\
\left(V^{2}+U^{-3}\right) \oplus U(U V) & \left(V^{2}+U^{3}+V^{3}\right) \oplus U(U V) \\
\left((U V) V+V^{2}\right) \oplus V & \left(U^{3}+U^{2} V+V^{3} \oplus V\right. \\
\left((U V) V+V^{2}\right) \oplus U^{2} & \left(U^{3}+U^{2} V+V^{3} \oplus U^{2}\right. \\
\left((U V) V+V^{2}\right) \oplus U^{2}(U V) & \left(U^{3}+U^{2} V+V^{3} \oplus U(U V)\right. \\
\left(U^{2} V+(U V) V\right) \oplus V & \left(U^{3}+U^{2} V\right) \oplus V \\
\left(U^{2} V+(U V) V\right) \oplus U^{2} & \left(U^{3}+U^{2} V\right) \oplus U^{2} \\
\left(U^{2} V+(U V) V\right) \oplus U(U V) & \left(U^{3}+U^{2} V\right) \oplus U(U V) \\
&
\end{array}
$$

Demonstraçio: Vimos na Seçào 2.1 que os P-subespaços marcados com * sào invariantes. Utilizando estes P-subespaços e o Corolário 2.9 obtemos que os P-subespaços do grupo I têm dimensào invariante. Os subespaços do grupo II têm dimensão invariante, pois estào contidos em $V$. Cada P-subespaço do Grupo II I é uma soma de um P-subespaço do grupo $I$ com um do grupo $I I$; os que não estão marcados com * não são invariantes, visto que não satisfazem a primeira condição da parte (b) do Teorema 2.3. Nos exemplos 2.2. 2.3 e 2.4 vimos que os P-subespaços de grupo IV nào têm dimensào invariante. Os subespaços do grupo $V$ sào somas dos subespaços do grupo $I V$ com subespaços do grupo $I I$ e. portanto, não têm dimensào invariante. 


\section{Capítulo 3}

\section{Invariância de $\mathbf{P}$-subespaços em outras álgebras báricas}

Consideraremos neste capítulo algumas classes de álgebras báricas $(A, \omega)$ sobre um corpo $K$ que possuem uma decomposição de Peirce análoga à das álgebras de Bernstein. Na Seção 3.2 estudaremos um par $(A, \lambda)$, onde $\lambda$ é uma forma linear. Logo, $(A, \lambda)$ nào é necessariamente uma álgebra bárica, mas mesmo assim possui uma decomposição de Peirce da forma $A=K \epsilon \pm U \Psi V:$ no caso em que $\lambda\left(A^{2}\right)=0$, a decomposiçào é feita com relaçào a um elemento nilpotente.

Para estas álgebras usamos a mesma definiçào de P-subespaço dada para álgebras de Bernstein e adotamos as mesmas notaçòes: $p=p(U, V), \partial m, o(p), \mathcal{F}$, etc.

Na Seção 3.1 consideramos a questão da invariância de P-subespaços nas train álgebras de posto 3. que sào álgebras báricas (A,w) sobre um corpo $K$ satisfazendo a equaçào $x^{3}-$ $(1+\gamma) \omega(x) x^{2}+\gamma \omega(x)^{2} x=0$ com $\gamma \in K$. A classe das train álgebras de posto 3 é outra classe que contém as álgebras de Bernstein-Jordan. De fato. fazendo $\gamma=0$ na equaçào anterior obtemos $x^{3}=\omega(x) x^{2}$. Pela Proposição 0.3. té Bernstein-.Jordan.

As álgebras consideradas na Seçào 3.2 satisfazem a equaçào $x^{3}=\lambda(x) x^{2}$. Elas sào. portanto. uma generalizaçào das álgebras de Bernstein-Jordan.

Nas seçòes 3.3 e 3.4 estudamos os P-subespaços de álgebras báricas satisfazendo as equaçòes $\left(x^{2}\right)^{2}=\omega(x) \cdot x^{3} \mathrm{e}\left(x^{2}\right)^{2}=\omega\left(x^{3}\right) x$, respectivamente. 


\subsection{Train álgebras de posto 3}

Seja $(A, \omega)$ uma álgebra bárica de dimensào finita sobre um corpo $K \operatorname{com} \operatorname{car}(K) \neq 2$. Dizemos que $(A, \omega)$ é uma train álgebra se

$$
a^{n}+\gamma_{1} \omega(a) a^{n-1}+\ldots+\gamma_{n-1} \omega(a)^{n-1} a=0
$$

para todo $a \in A$. Os escalares $\gamma_{1}, \ldots, \gamma_{n-1}$ satisfazem $1+\gamma_{1}+\ldots+\gamma_{n-1}=0$, pois se $a \in A$ é tal que $\omega(a) \neq 0$, então

$$
\begin{aligned}
0 & =\omega\left(a^{n}+\gamma_{1} \omega(a) a^{n-1}+\ldots+\gamma_{n-1} \omega(a)^{n-1} a\right) \\
& =\omega(a)^{n}\left(1+\gamma_{1}+\ldots+\gamma_{n-1}\right)
\end{aligned}
$$

Um polinômio da forma

$$
p(x)=x^{n}+\gamma_{1} \omega(x) x^{n-1}+\ldots+\gamma_{n-1} \omega(x)^{n-1} x
$$

cujos coeficientes satisfazem $1+\gamma_{1}+\ldots+\gamma_{n-1}=0$ é chamado de train polinômio de grau $n$. Em uma train álgebra existe um único train polinômio $p(x)$ de menor grau que satisfaz $p(a)=0$ para todo $a \in A$. O grau deste polinômio é chamado de posto de $A$. Uma train álgebra de posto $n$ satisfaz $x^{n}=0$ para todo $x \in N$. De onde concluímos que $\omega$ é o único homomorfismo de $A$ em $K$. Dizemos entào que $Y$ é o núcleo de $A$.

As train álgebras de posto 3 satisfazem uma identidade da forma

$$
x^{3}-(1+\gamma) w(x) x^{2}+\gamma w(x)^{2} x=0
$$

com ๆ em $K$. Não consideraremos as train álgebras de posto 3 para as quais $\gamma=\frac{1}{2}$. Quando $\gamma \neq \frac{1}{2}$ a train álgebra correspondente tem elementos idempotentes de peso 1 . De fato, se $d \in A$ e $\omega(d)=1$ entào $e=\frac{1}{1-2 \gamma}\left(d^{2}-2 \gamma d\right)$ satisfaz $\epsilon^{2}=\epsilon$ e $\omega(\epsilon)=1$ (ver [14]).

Para cada idempotente $e$ de peso 1 , temos a seguinte decomposição

$$
A=K \epsilon \oplus N=K^{*} \epsilon \div V_{\epsilon} \oplus V_{\epsilon}
$$

chamada decomposição de Peirce de $A$ relativa a $\epsilon$, onde

$$
\begin{aligned}
& U_{e}=\{u \in A: 2 \epsilon u=u\} \\
& V_{\epsilon}=\{v \in A: \epsilon v=\gamma v\}
\end{aligned}
$$

sào os subespaços próprios do operador linear $L_{\epsilon}: V \rightarrow V$ definido por $L_{\epsilon}(x)=\epsilon x$, correspondente aos valores próprios distintos $\frac{1}{2} \mathrm{e}_{j}$. Tais subespaços satisfazem as relaçòes

$$
U_{\epsilon}^{2} \subseteq V_{\epsilon} \quad U_{\epsilon} V_{\epsilon} \subseteq U_{\epsilon}^{*} \quad V_{\epsilon}^{2}=0
$$


Todo elemento do núcleo $Y$ de uma train álgebra de posto 3 satisfaz

$$
x^{3}=0
$$

Portanto, os elementos do núcleo de uma train álgebra de posto 3 também satisfazem as identidades (30) e (31). Usando (31) e (3.2) obtemos

$$
\left(x^{2}\right)^{2}=0
$$

para qualquer $x \in N$. Assim, as identidacles (3) e (4) também são satisfeitas para os elementos de.$V$.

Agora. se $u \in U_{\epsilon}$ e $v \in V_{\epsilon}$. entào segue de (3.1) e (31) que

$$
\begin{aligned}
v^{2} & =0 \\
u(u v) & =0 \\
v(v u) & =0
\end{aligned}
$$

para quaisquer $u \in U_{e}$ e $v \in V_{\epsilon}$.

O conjunto dos idempotentes de $A$ de peso 1 é dado por

$$
I p(. A)=\left\{e+u+\theta u^{2} ; u \in U_{\epsilon}\right\}
$$

onde $\theta=(1-2 \gamma)^{-1}$. Para um outro idempotente $f=\epsilon+u_{0}+\theta u_{0}^{2}$ de $A$, com $u_{0} \in L_{\epsilon}^{-}$, temos a decomposiçào de Peirce $f=K^{\circ} U_{f} \pm V_{f}$ associada a $f$, onde

$$
\begin{aligned}
& U_{f}=\left\{u+2 \theta u_{0} u ; u \in V_{\epsilon}\right\} \\
& V_{f}=\left\{v-2 \theta u_{0} v: v \in V_{\epsilon}\right\}
\end{aligned}
$$

e as aplicaçòes lineares $\sigma: U_{\epsilon} \rightarrow U_{f}, \tau: V_{\epsilon} \rightarrow V_{f}, U_{\epsilon} \rightarrow V_{\epsilon} e_{\tau}: A \rightarrow A$ definidas por

$$
\begin{aligned}
\sigma(u) & =u+2 \theta u_{0} v \\
\tau(v) & =v-2 \theta u_{0} v \\
\psi(u) & =u-2 \theta^{2} u_{0}^{2} u \\
\hat{\gamma}(a \epsilon+u+v) & =a \epsilon+\sigma(u)+\tau(v)
\end{aligned}
$$

onde $u \in U$ e $v \in V$. sào isomorfismos de espaços vetoriais. A proposiçào seguinte é análoga a Proposiçào 2.3. 
Proposição 3.1 Seja $A=K \in \oplus\left\lfloor\oplus V\right.$ uma train álgebra de posto 3 . As funções $\sigma, \tau \in \iota^{\circ}$ satisfazem as relações

$$
\begin{aligned}
\sigma\left(u_{1}\right) \sigma\left(u_{2}\right) & =\tau\left(u_{1} u_{2}\right) \\
\sigma(u) \tau(v) & =\sigma(u \cdot(u) v)
\end{aligned}
$$

para quaisquer $u, u_{1}, u_{2} \in U \in v \in V$.

Agora, procedendo de maneira inteiramente análoga ao que foi feito nas seções 2.3 e 2.4 obtemos, quanto a invariância de P-subespaços. os mesmos resultados que foram obtidos para álgebras de Bernstein-Jordan. Reunimos a seguir. num único enunciado. tais resultados.

Teorema 3.1 Seja t uma train álgebra de posto 3 . Então

(1) Todo P-subespaço $p$ de A satisfaz $y\left(p_{e}\right)=p_{f}$. Em particular, todo P-subespaço tem dimensào invariante.

(2) Para todo P-subespaço $p$ de A as afirmaçós seguintes são equivalentes:

(2.1) p é invariante;

(2.2) lip $\subseteq p$ :

(2.3) p é ideal;

(3) O conjunto dos P-subespaços invariantes de $\perp$ é dado por $\{p+[p ; p \in \mathcal{P}\}$.

\section{2 Álgebras satisfazendo $x^{3}=\lambda(x) x^{2}$}

Estudaremos nesta seçào o problema da invariância de P-subespaços de uma álgebra At sobre um corpo $i$ satisfazendo a identidade

$$
x^{3}=\lambda(x) x^{2}
$$

onde $\lambda$ é uma forma linear. Assim, todo elemento do núcleo de $\lambda$ satisfaz

$$
x^{3}=0
$$

Portanto. temos também as linearizaçòes desta identidade

$$
\begin{aligned}
x_{1}^{2} x_{2}+2 x_{1}\left(x_{1} x_{2}\right) & =0 \\
\left(x_{1} x_{2}\right) x_{3}+\left(x_{2} x_{3}\right) x_{1}+\left(x_{3} x_{1}\right) x_{2} & =0
\end{aligned}
$$


A existência de idempotentes não nulos em $A$ depende de $\lambda$. Por exemplo, se $\lambda$ é tal que $\lambda\left(x^{2}\right)=0$ para todo $x \in A$ então $A$ não possui idempotentes não nulos. Com efeito, se $\epsilon=e^{2} \in \mathcal{A}$ então $e=\epsilon^{3}=\lambda(\epsilon) \epsilon^{2}=0$. Exemplos desta situaçào podem ser encontrados em [12]. O estudo desta álgebra é entào dividido em dois: quando $\lambda\left(A^{2}\right)=0$ e quando $\lambda\left(A^{2}\right) \neq 0$.

\subsubsection{O caso $\lambda\left(A^{2}\right)=0$}

Nesta seçào, consideraremos que $\operatorname{car}\left(K^{\prime}\right) \neq 2,3$ e que o par $(A, \lambda)$ satisfaz

$$
\lambda\left(A^{2}\right)=0
$$

Estas álgebras sào estudadas em [12]. Faremos aqui um resumo das suas principais propriedades.

Existe pelo menos um elemento nilpotente $z$ em $A$ tal que $z^{2}=0$ e $\lambda(z)=1$. Cada um destes elementos determina uma decomposição $A=K \approx \oplus U \Psi V$ chamada de decomposição de Peirce de A relativa a $z$, onde $U$ e $V$ sào subespaços que satisfazem $N=U \oplus V$ e

$$
\begin{aligned}
& U=\{u \in N ; z u=0\} \\
& V=\{v \in N ; z v=v\}
\end{aligned}
$$

Além disso, temos as seguintes relaçòes

$$
U V \subseteq U, \quad U^{2} \subseteq V, \quad V^{2}=0
$$

Quando houver necessidade de especificar a que elemento $z$ estào associados os subespaços $U$ e $V$ escreveremos $U_{z}$ e $V_{z}$.

Os elementos $u \in U$ e $v \in V$ satisfazem as identidades

$$
u(u v)=(u v) v=v^{2}=0
$$

Da linearização de (3.8), obtemos

$$
\begin{aligned}
u_{1}\left(u_{2} v^{\prime}\right)+u_{2}\left(u_{1} v\right) & =0 \\
\left(u v_{1}\right) v_{2}+\left(u v_{2}\right) v_{1} & =0 \\
v_{1} v_{2} & =0
\end{aligned}
$$

para quaisquer $u, u_{1}, u_{2} \in U$ e $v, v_{1} \in I^{\circ}$.

O próximo lema segue imediatamente de (3.9) e (3.10). 
Lema 3.1 Se $X . X_{1}, X_{2} \subseteq U \in W, W_{1}, W_{2} \subseteq V$ são subespaços de $A=K z \oplus U \oplus V$. então

(a) $\mathrm{X}_{1}\left(\mathrm{X}_{2} W^{\circ}\right)=\mathrm{X}_{2}\left(\mathrm{X}_{1} W\right)$;

(b) $W_{1}\left(W_{2} X^{r}\right)=W_{2}\left(W_{1} X^{r}\right)$.

A linearizaçào de (3.3), para $x, y \in A$, é dada por

$$
x^{2} y+2 x(x y)=\lambda(y) x^{2}+2 \lambda(x) x y
$$

Substituindo-se, nesta identidade, $y$ por $x^{2}$ temos que

$$
\left(x^{2}\right)^{2}=0
$$

para todo $x \in X$. Obtemos das linearizaçòes de (3.13) as seguintes identidades:

$$
\begin{aligned}
x_{1}^{2}\left(x_{1} x_{2}\right) & =0 \\
x_{1}^{2}\left(x_{2} x_{3}\right)+2\left(x_{1} x_{2}\right)\left(x_{1} x_{3}\right) & =0 \\
\left(x_{1} x_{2}\right)\left(x_{3} x_{4}\right)+\left(x_{1} x_{3}\right)\left(x_{2} x_{4}\right)+\left(x_{1} x_{4}\right)\left(x_{2} x_{3}\right) & =0
\end{aligned}
$$

para quaisquer $x_{1}, x_{2}, x_{3} \in N$.

A proposição seguinte é o Corolário 2.3 de [12].

Proposição 3.2 As seguintes condiçôes scio equicalentes:

(a) A forma linear $\lambda$ nào é única:

(b) Á é uma zero álgebra:

(c) $V=0$.

Portanto. o caso em que $\lambda$ não é única não tem interesse. Assim, vamos considerar que a forma linear $\lambda$ é única e diremos que $N$ é o núcleo de $t$.

O conjunto dos elementos nilpotentes de $A$ é dado por

$$
\operatorname{Nilp}(A)=\left\{z+u-\frac{1}{2} u^{2} z: u \in U_{z}\right\}
$$

Se $f=z+u-\frac{1}{2} u^{2}$ é outro elemento nilpotente de $A$ com $u \in U_{z}$, entào

$$
\begin{aligned}
U_{f} & =\left\{x-u x: x \in C_{z}\right\} \\
V_{f} & =\left\{v+u v: v \in V_{z}\right\}
\end{aligned}
$$


As funçòes lineares $\sigma: U_{z} \rightarrow U_{f}, \tau: V_{z} \rightarrow V_{f}$ e $\nu: A \rightarrow A$ definidas por

$$
\begin{aligned}
\sigma(x) & =x-u x \\
\tau(v) & =v+u v \\
\varphi(\alpha z+x+v) & =\alpha f+\sigma(x)+\tau(v)
\end{aligned}
$$

são isomorfismos de espaços vetoriais. A funçào $\varphi$ é chamada de transformação de Peirce de $A$ associada a $z$ e a $u$. Em [12] os autores estudam várias propriedades das transformações de Peirce.

A demonstração da próxima proposição é inteiramente análoga a da Proposição 2.6.

Proposição 3.3 Para todo P-subespaço $p$ de At tem-se $V p \subseteq p$.

A proposição seguinte corresponde à Proposiçào 2.3 e pode ser demonstrada de maneira análoga utilizando-se a funçào $\psi: U_{z} \rightarrow U_{z}$ definida por $\psi(x)=x-\frac{1}{2} u^{2} x$. Mas faremos aqui outra demonstraçào.

Proposição 3.4 Sejam $\mu, \mu_{1} . \mu_{2} \subseteq U \in \nu . \nu_{1}, \nu_{2} \subseteq V P$-monômios de $A=K z \boxplus U \oplus V$. Então toda transformaçào de Peirce $\varphi$ de A satisfaz:

(a) $\varphi\left(\mu_{1} \mu_{2}\right)=\varphi\left(\mu_{1}\right) \varphi\left(\mu_{2}\right)$ :

(b) $\hat{\varphi}\left(\nu_{1} \nu_{2}\right)=\varphi\left(\nu_{1}\right) \varphi\left(\nu_{2}\right)=0$;

(c) $\varphi(\mu \nu)=\varphi(\mu) \varphi(\nu)$.

Demonstração: Seja $\varphi$ a transformaçào de Peirce de $A$ associada a $z \in N$ ilp $(A)$ e a $u_{0} \in U$. Se $u_{1}, u_{2} \in U$ e $v_{1}, v_{2} \in V$ entào

$$
\begin{aligned}
& \varphi\left(u_{1} u_{2}\right)=\varphi\left(u_{1}\right) \varphi\left(u_{2}\right) \\
& \varphi\left(v_{1} v_{2}\right)=\varphi\left(v_{1}\right) \varphi\left(v_{2}\right)
\end{aligned}
$$

pela Proposição 3.2 de [12]. Destas identidades decorrem (a) e (b). Resta mostrar a parte (c). Segue clas identidades (3.9), (3.11) e (3.15) que

$$
\varphi(u) \varphi(v)=\varphi(u v)+\frac{1}{2} u_{0}^{2}(u v)
$$

para quaisquer $u \in U$ e $v \in V$. Mostremos agora que se $u \in \mu$ e $v \in \nu$ entào

$$
u_{0}^{2}(u v) \in \hat{T}(\mu \nu) \cap \hat{T}(\mu)_{T}(\nu)
$$


Pela Proposição 3.3. temos que $u_{0}^{2}(u v) \in V(\mu \nu) \subseteq \mu \nu$. Usando (3.4) e (3.9), obtemos a identidade $u_{0}\left(u_{0}^{2}(u v)\right)=0$. Portanto, $u_{0}^{2}(u v)=\varphi\left(u_{0}^{2}(u v)\right)$. De onde segue que $u_{0}^{2}(u v) \in$ $\varphi(\mu \nu)$. Por outro lado. $u_{0}^{2}\left(u c^{\circ}\right)=-\left(u_{0}^{2} u\right) v$ por (3.10). Assim, $u_{0}^{2}\left(u v^{*}\right)=-\varphi\left(u_{0}^{2} u\right) \varphi(v)$, visto que $u_{0}\left(u_{0}^{2} u\right)=\left(u_{0} v\right)\left(u_{0}^{2} u\right)=0$ por $(3.9)$ e (3.16). Portanto, usando novamente a Proposiçào 3.3 , temos

$$
u_{0}^{2}(u \nu) \in \varphi(V \mu) \varphi(\nu) \subseteq \vartheta(\mu) \varphi(\nu)
$$

o que conclui a prova de (3.18). Agora, (3.17) e (3.18) implicam que $\varphi(\mu \nu)=\varphi(\mu) \varphi(\nu)$.

Os dois lemas seguintes também são análogos a resultados similares das seções anteriores.

Lema 3.2 Se $h \subseteq V$ é um P-subespaço de $\mathrm{A}$ com $o(p) \geq 2$. então existe um P-subespaço $g \subseteq U$ tal que $h=U g$.

Lema 3.3 Todo P-subespaço de A satisfaz $U(U p) \subseteq p$.

Agora. do mesmo modo como fizemos na seção anterior, reuniremos todos os resultados sobre invariância de um P-subespaço em um único enunciado. cuja demonstração é completamente análoga à que foi feita nas seçòes 2.3 e 2.4.

Teorema 3.2 Seja $(A, \lambda)$ satisfazendo $\lambda\left(A^{2}\right)=0$ onde $\lambda$ é uma forma linear em $A$. Então

(1) Todo P-subespaço $p$ de A satisfaz $\varphi\left(p_{\varepsilon}\right)=p_{f}$. Em particular todo P-subespaço tem dimensão invariante.

(2) Para todo P-subespaço $p$ de A as afirmaçòes seguintes são equivalentes:

(2.1) p é invariante:

(2.2) $l p \subseteq p$;

(2.3) pé ideal:

(3) O conjunto dos P-subespaços invariantes de té dado por $\{p+l p ; p \in \mathcal{P}\}$. 


\subsubsection{O caso $\lambda\left(A^{2}\right) \neq 0$}

Uma generalização das álgebras de Bernstein-Jordan são as álgebras de Bernstein-Jordan fracas definidas em [ 7$]$. Segue do Teorema 2.8 de [i] que uma $K$-álgebra $A$ é BernsteinJordan fraca se, e somente se, existe uma forma linear $\lambda$ tal que, para todo $x \in A$,

$$
\left\{\begin{array}{l}
x^{3}=\lambda(x) \cdot x^{2} \\
\lambda\left(A^{2}\right) \neq 0
\end{array}\right.
$$

Em [i] também é dado um exemplo de uma tal álgebra que nào é associativa, não é bárica e nào é Jordan.

Enunciaremos a seguir as propriedades das quais necessitaremos para o estudo dos Psubespaços nas álgebras que satisfazem (3.19). Maiores detalhes podem ser encontrados em $[\bar{\imath}]$.

Em A sempre existe um idempotente não nulo $\epsilon$, tal idempotente determina uma decomposição de $A$ da forma $A=K e \oplus \operatorname{ker} \lambda=K \epsilon \oplus U \oplus V$, chamada de decomposição de Peirce de $A$. onde $N=\operatorname{ker} \lambda=U \oplus V$ e

$$
\begin{aligned}
& U=\left\{u \in N: e u=\frac{1}{2} u\right\} \\
& V=\{v \in N: \epsilon v=0\}
\end{aligned}
$$

Os subespaços $U$ e $V$ satisfazem

$$
U V \subseteq U, \quad I^{2} \subseteq V \quad V^{2} \subseteq K \epsilon
$$

Escreveremos $U_{\epsilon}$ e $V_{e}$ quando quizermos deixar claro que a decomposiçào de Peirce foi feita usando-se o particular idempotente $e$.

Observemos que $N$ nào é necessariamente um ideal. O Teorema 2.9 de [i] nos diz que $N$ é um ideal se, e somente se. $(A, \lambda)$ é Bernstein-Jordan.

Nas álgebras que satisfazem (3.19) não podemos escrever todos os P-subespaços na forma $p=g \oplus h \operatorname{com} g \subseteq U$ e $h \subseteq V$. pois podemos ter. por exemplo, $p=h \epsilon \pm g \Phi h$.

Para os elementos $u, u_{1}, u_{2} \in U$ e $v \in V$ temos as seguintes identidades.

$$
\begin{aligned}
u^{2} v & =0 \\
u_{1}\left(u_{2} v^{2}\right)+u_{2}\left(u_{1} v\right) & =0
\end{aligned}
$$

Segue de $(3.20)$ que $L^{2} V=0$ e

$$
\left(u_{1} u_{2}\right) v=0
$$


$\mathrm{O}$ conjunto dos elementos idempotentes nào nulos de $A$ é dado por

$$
\operatorname{Ip}(A)=\left\{e+u+u^{2} ; u \in U_{\epsilon}\right\}
$$

e se $f=\epsilon+u+u^{2}$ é outro idempotente de $A$, com $u \in U_{\epsilon}$, entào

$$
\begin{aligned}
U_{f} & =\left\{x+2 u x ; x \in U_{\epsilon}\right\} \\
V_{f} & =\left\{v-2 u v ; v \in V_{e}\right\}
\end{aligned}
$$

As funçòes lineares $\sigma: U_{e} \rightarrow U_{f}, \tau: V_{e} \rightarrow V_{f}$ e $\varphi: A \rightarrow A$ definidas por

$$
\begin{aligned}
\sigma(x) & =x+2 u x \\
\tau(v) & =v-2 u v \\
\varphi(\alpha e+x+v) & =\alpha f+\sigma(x)+\tau(v)
\end{aligned}
$$

são isomorfismos de espaços vetoriais e y é chamada de transformação de Peirce de $A$ associada a $\epsilon$ e a $u$.

Foi provado em [7] que os $\mathrm{P}$-subespaços $U, V, U V, U^{2}$ e $V^{2}$ têm dimensão invariante. Provaremos, procedendo como nas seções anteriores, que isto ocorre para todos os P-subespaços de $A$.

Como $V^{2} \subseteq K \epsilon$. existem duas possibilidades: $V^{2}=0$ ou $V^{2}=K \epsilon$. Se $V^{2}=0$ entào $N$ é ideal de $A$ e, portanto, $(A, \lambda)$ é Bernstein-Jordan. Neste caso, o estudo da invariância já foi feito. Assim, de agora em diante, assumiremos que $V^{2}=K \epsilon$.

Inicialmente observemos que se $m$ é um P-monômio com $\partial m \geq 3$ e $m \subseteq V^{2}$ então $m\left(U_{\epsilon}, V_{\epsilon}\right)=0$. De fato. existem $\nu_{1}, \nu_{2} \subseteq V$ com, por exemplo. $\partial \nu_{2} \geq 2$ tal que $n=\nu_{1} \nu_{2}$. Temos que $\nu_{2} \subseteq U^{2}$, visto que $\partial \nu_{2} \geq 2$. Portanto, $m \subseteq U^{2} V=0$. Além disso, para quaisquer P-subespaços $g \subseteq U$ e $h \subseteq V$ temos

$$
\begin{aligned}
& g V^{2}=g K e=g \\
& h V^{2}=h K e=0
\end{aligned}
$$

Assim, dado um P-monômio $m$ com $\partial m \geq 2$, para o restante desta seçào, basta considerar somente três possibilidades: $m=\mu \nu, m=\mu_{1} \mu_{2}$ ou $m=V^{2}=k \epsilon$. onde $\mu_{1}, \mu_{1}, \mu_{2} \subseteq U$ e $\nu \subseteq \mathrm{V}$ são $\mathrm{P}$-monômios de grau menor ou igual a $\partial \mathrm{m}$.

Lema 3.4 Para todo P-subespaço p de uma álgebra que satisfaz (3.19) tem-se $U^{2} p \subseteq p$.

Demonstraçrio: Basta provar o resultado para um $\mathrm{P}$-monômio genérico $m$. Temos que

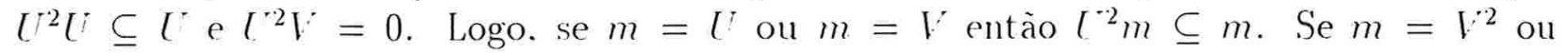


$m=\mu_{1} \mu_{2}$. onde $\mu_{1}, \mu_{2} \subseteq U$ sào $\mathrm{P}$-monômios de $A$ então $\left[I^{2} m=0\right.$. Falta o caso em que $m=\mu \nu$, onde $\mu \subseteq U$ e $\nu \subseteq V$ são P-monômios. Temos que $U^{2} m$ é gerado por elementos da forma $u_{1}^{2}\left(u_{2} v\right)$ com $u_{1} \in U, u_{2} \in \mu$ e $v \in \nu$. Segue de (3.6) e (3.20) que

$$
u_{1}^{2}\left(u_{2} v\right)=-\left(u_{1}^{2} u_{2}\right) v \in\left(L^{-2} \mu\right) \nu
$$

Agora, usando indução em $\partial m$, obtemos $I^{2} m \subseteq m$.

Proposição 3.5 Sejam $\mu, \mu_{1}, \mu_{2} \subseteq U$ and $\nu \subseteq V$ P-monômios de uma álgebra que satisfaz (3.19). Então toda transformação de Peirce y de A satisfaz

(a) $\varphi\left(\mu_{1} \mu_{2}\right)=\varphi\left(\mu_{1}\right) \varphi\left(\mu_{2}\right)$

(b) $\varphi\left(V^{2}\right)=\varphi(V) \varphi(V)$

(c) $\varphi(\mu \nu)=\varphi(\mu) \varphi(\nu)$

Demonstraçào: Seja $\varphi$ a transformaçào de Peirce de $A$ associada a $e \in \operatorname{Ip}(A)$ e a $u_{0}$. É claro. a partir da Proposiçào 4.4 de [i], que $\varphi\left(\mu_{1} \mu_{2}\right)=\varphi\left(\mu_{1}\right) \varphi\left(\mu_{2}\right)$ e $\varphi\left(V_{\epsilon}^{2}\right)=\varphi\left(V_{\epsilon}\right) \varphi\left(V_{e}\right)$. Agora. sejam $u \in \mu$ e $v \in \nu$. As identidades (3.5). (3.6) e (3.22) implicam que $\left(u_{0} u\right)\left(u_{0} v\right)=\frac{1}{2}\left(u_{0}^{2} u\right) v$. Então, usando (3.21) e (3.22), obtemos

$$
\varphi(u) \varphi(v)=\varphi(u v)-2\left(u_{0}^{2} u\right) v
$$

Segue de (3.4) e $(3.21)$ que

$$
u_{0}\left(u_{0}^{2} u\right)=0
$$

para qualquer $u \in U_{\epsilon}$. De (3.6) e (3.20) temos que $u_{0}\left(\left(u_{0}^{2} u\right) v\right)=-u_{0}\left((u v) u_{0}^{2}\right)$. Logo. $u_{0}\left(\left(u_{0}^{2} u\right) v\right)=0$ por $(3.24)$. Portanto, $\left(u_{0}^{2} u\right) v=\tau\left(\left(u_{0}^{2} u\right) v\right)$. Agora usando o Lema 3.4 temos

$$
\left(u_{0}^{2} u\right) v \in\left(I^{2} \mu\right) \nu \subseteq \mu \nu
$$

Logo $\left(u_{\mathrm{c}}^{2} u\right) v \in \hat{\varphi}(\mu \nu)$. Por outro lado, de (3.24) temos que

$$
\varphi\left(u_{0}^{2} u\right) \varphi(v)=\left(u_{0}^{2} u\right) v-2\left(u_{0}^{2} u\right)\left(u_{0} v\right)
$$

Agora. segue de (3.6), (3.20) e (3.24) que

$$
\left(u_{0}^{2} u\right)\left(u_{0} v\right)=-u_{0}\left(v\left(u_{0}^{2} u\right)\right)-v\left(\left(u_{0}^{2} u\right) u_{0}\right)=0
$$

Assim, $\left(u_{0}^{2} u\right) v=\varphi\left(u_{0}^{2} u\right) \varphi(v)$. Portanto, usando novamente o Lema 3.4, temos que $\left(u_{0}^{2} u\right) v \in$ $\varphi(\mu) \varphi(\nu)$. Concluímos que $\left(u_{0}^{2} u\right) v \in \varphi(\mu \nu) \cap \varphi(\mu) \varphi(\nu)$. Portanto. segue de (3.23) que $\varphi(\mu)_{\varphi}(\nu)=\varphi(\mu \nu)$.

Agora. da mesma maneira como foi feito nas seçòes anteriores pode-se demonstrar o seguinte teorema. 
Teorema 3.3 Todo P-subespaço $p$ de A satisfaz $\varphi\left(p_{e}\right)=p_{f}$. Em particular todo Psubespaço tem dimensão invariante.

Para as álgebras que satisfazem (3.19) com $V^{2}=$ he não podemos demonstrar um resultado análogo ao da Proposição 2.7. Por exemplo, o P-subespaço $U \in V$ é invariante, pois $U \oplus V=\operatorname{ker} \lambda$, mas $U \oplus V$ nào é um ideal. Entretanto, para P-subespaços da forma $p=g \oplus h$ com $g \subseteq U$ e $h \subseteq V$, temos um resultado análogo ao Teorema 2.2 com demonstração similar.

Teorema 3.4 Seja $A=K e \oplus U \oplus V$ uma álgebra satisfazendo (3.19) com $V^{2}=K$ lie. Então um P-subespaço da forma $p=g \oplus h$. onde $g \subseteq U$ e $h \subseteq V$ é invariante se. e somente se. I $p \subseteq p$.

C'om os dois lemas seguintes poderemos caracterizar alguns ideais que são P-subespaços.

Lema 3.5 Se $A=K e \oplus U \oplus V$ é uma álgebra satisfazendo (3.19) com $V^{2}=K \epsilon \in U \neq 0$. entào $V g=g$, para qualquer P-subespaço g contido $\mathrm{em} U$.

Demonstração: Basta provar para um P-monômio $m \subseteq U$. Faremos induçào em $\partial m$. Se $\partial m=1$, então $m=U$. Temos que $V U=U$, pela Proposição 3.6 de [i]. Suponhamos que $\partial m \geq 2$. Logo, $m=\mu \nu$ onde $\mu \subseteq U$ e $\nu \subseteq V$ sào P-monômios com $\partial \mu, \partial \nu \leq \partial m$. Se $\nu=V$ então $V(\mu \nu)=V \mu=m$. pela hipótese de indução. Suponhamos agora que $\nu=\mu_{1} \mu_{2}$ onde $\mu_{1}, \mu_{2} \subseteq U$ săo $\mathrm{P}$-monômios. Temos que $V\left(\mu\left(\mu_{1} \mu_{2}\right)\right)$ é gerado por elementos da forma $v\left(u\left(u_{1} u_{2}\right)\right)$, onde $v \in V, u \in \mu, u_{1} \in \mu_{1}$, e $u_{2} \in \mu_{2}$. Segue de (3.6) e (3.22) que $v\left(u\left(u_{1} u_{2}\right)\right)=-\left(u_{1} u_{2}\right)(u v)$. Portanto. $V(\mu \nu)=\nu(V \mu)=m$.

Lema 3.6 Sejam $A=K e \oplus U \oplus V$ uma álgebra que satisfaz (3.19) com $V^{2}=$ Le e $U \neq 0$. e $p$ um P-subespaço de A da forma $p=g \oplus h$, onde $g \subseteq U$. Se $U p \subseteq p$ e o $(h) \geq 2$ então $p$ é um ideal.

Demonstração: Temos que $e p=g \subseteq p$. e $U p \subseteq p$ pela hipótese. Agora. $o(h) \geq 2$ implica que $h \subseteq U^{2}$. Entào $V h \subseteq U^{2} V=0$. Assim, $V p=V g=g \subseteq p$.

Corolário 3.1 Sejam $A=K e \mp U \mp V$ uma álgebra que satisfaz (3.19) tal que $V=$ lie. $U \neq 0$ e $p$ um P-subespaço da forma $p=g \pm h$ onde $g \subseteq U$. Se $o(h) \geq 2$. então as seguintes condições são equivalentes:

(a) pé invariante;

(b) $L^{\top} p \subseteq p$ :

(c) pé um ideal de. 1 . 


\section{3 Álgebras satisfazendo $\left(x^{2}\right)^{2}=\omega(x) x^{3}$}

Seja $K$ um corpo com $\operatorname{car}\left(K^{\prime}\right) \neq 2$ e 3 . Estudaremos agora álgebras báricas $(A, \omega)$ sobre II que satisfazem

$$
\left(x^{2}\right)^{2}=\omega(x) x^{3}
$$

para todo $x \in A$. Há um sucinto estudo destas álgebras em [1].

Segue de (3.25) que

$$
\left(x^{2}\right)^{2}=0
$$

para todo $x \in N=$ ker w. Linearizando (3.26), obtemos

$$
\begin{aligned}
x_{1}^{2}\left(x_{1} x_{2}\right) & =0 \\
x_{1}^{2}\left(x_{2} x_{3}\right)+2\left(x_{1} x_{2}\right)\left(x_{1} x_{3}\right) & =0
\end{aligned}
$$

onde $x_{1}, x_{2}, x_{3}, x_{4} \in X$.

As duas primeiras linearizações de (3.25) são dadas por

$$
\begin{aligned}
4 x^{2}(x y) & =\omega(y) x^{3}+\omega(x)\left(x^{2} y+2 x(x y)\right) \\
4 x^{2}(y z)+S(x y)(x z) & =2 \omega(x)((x y) z+(y z) x+(z x) y) \\
& +\omega(y)\left(x^{2} z+2 x(x z)\right)+\omega(z)\left(x^{2} y+2 x(x y)\right)
\end{aligned}
$$

onde $x, y, z \in A$. Fazendo $y=z$ em (3.30), obtemos

$$
4(x y)^{2}+2 x^{2} y^{2}=\omega(x)\left(x y^{2}+2 y(y \cdot x)\right)+w(y)\left(x^{2} y+2 x(x y)\right)
$$

Mostraremos que $\operatorname{Ip}(A)=\left\{z^{3} \in A ; \omega(z)=1\right\}$. De fato. seja $z \in A$ tal que $\omega(z)=1$. Portanto, $\left(z^{2}\right)^{2}=z^{3}$. Logo, segue de (3.25), fazendo $x=z^{2}$. que $\left(z^{3}\right)^{2}=\left(z^{2}\right)^{3}$. Assim. $\left(z^{3}\right)^{2}=z^{2}\left(z^{2}\right)^{2}$. Portanto,

$$
\left(z^{3}\right)^{2}=\left(z^{2}\right)^{3}=z^{2} z^{3}
$$

Substituindo $x$ por $z$ e $y$ por $z^{2}$ em (3.29) e em (3.31) obtemos

$$
\begin{aligned}
4 z^{2} z^{3} & =2 z^{3}+2 z^{4} \\
4\left(z^{3}\right)^{2} & =z^{3}+3 z^{4}
\end{aligned}
$$


Segue destas duas equações e de (3.32) que $z^{3}=z^{4}$. Substituindo esta igualdade em (3.33), obtemos $\left(z^{3}\right)^{2}=z^{3}$. Assim, $z^{3}$ é idempotente de $A$.

Sejam agora $e$ um idempotente de peso 1 de $A$ e $y, z \in N$. Fazendo $x=e$ em (3.30), temos

$$
4(e y)(e z)+e(y z)=(e y) z+(e z) y
$$

Fazendo $y=z$ em (3.34), temos

$$
4(\epsilon z)^{2}+\epsilon z^{2}=2(\epsilon z) z
$$

Se $x=\epsilon$ e $y=z$ em (3.29), então

$$
2 \epsilon(\epsilon z)=e z
$$

Novamente em (3.29), fazendo $x=z$ e $y=\epsilon$, obtemos

$$
4 z^{2}(\epsilon z)=z^{3}
$$

A primeira linearização desta identidade é dada por

$$
S(y z)(e z)+4 z^{2}(\epsilon y)=y z^{2}+2 z(z y)
$$

Seja $L_{e}: N \rightarrow N$ o operador linear definido por $L_{\epsilon}(z)=\epsilon z$. Segue de (3.36) que $L_{e}^{2}-$ $\frac{1}{2} L_{\epsilon}=0$. Assim $x\left(x-\frac{1}{2}\right)=0$ é um polinômio anulador de $L_{\epsilon}$. Portanto, $L_{\epsilon}$ é diagonalizável e os autovalores de $L_{e}$ estào contidos em $\left\{0, \frac{1}{2}\right\}$. Concluímos que $N=U_{e} \oplus V_{e}$, onde

$$
\begin{aligned}
& U_{e}=\left\{u \in A: e u=\frac{1}{2} u\right\} \\
& V_{e}=\{v \in A ; e v=0\}
\end{aligned}
$$

A decomposição $A=K e \oplus U_{\epsilon} \pm V_{\epsilon}$ é chamada de decomposição de Peirce de $A$.

Mostremos agora que

$$
U_{e} V_{e} \subseteq V_{e} \quad V_{\epsilon}^{2} \subseteq V_{e} \quad U_{e}^{2} \subseteq V_{e}
$$

Sejam $u \in U_{\epsilon}$ e $v \in V_{\epsilon}$. Tomando $z=u$ em (3.35), temos $\epsilon u^{2}=0$. Logo, $u^{2} \in V_{e}$. Assim, $U_{e}^{2} \subseteq V_{e}$. Analogamente, fazendo $y=u$ e $z=v$ em (3.34), tem-se $e(u v)=\frac{1}{2} u v$. De onde segue que $u v \in U_{e} V_{\epsilon}$. Logo. $U_{e} V_{e} \subseteq U_{e}$. Para mostrar que $V_{e}^{2} \subseteq V_{e}$, usamos mais uma vez $(3.3 .5) \mathrm{com} z=v$.

Em (3.3T), fazendo $z=u$ e depois $z=\imath$, obtemos

$$
u^{3}=i^{3}=0
$$


Tomando $y=u$ e $z=v$ em (3.38), temos

$$
u v^{2}=2(u v) v
$$

Analogamente, se $y=v$ e $z=u$ em (3.38), então

$$
u^{2} v=2 u(u v)
$$

As linearizções de (3.40). (3.41) e (3.42) são

$$
\begin{aligned}
u_{1}^{2} u_{2}+2 u_{1}\left(u_{1} u_{2}\right) & =0 \\
u_{1}\left(u_{2} u_{3}\right)+u_{2}\left(u_{3} u_{1}\right)+u_{3}\left(u_{1} u_{2}\right) & =0 \\
v_{1}\left(v_{2} v_{3}\right)+v_{2}\left(v_{3} v_{1}\right)+v_{3}\left(v_{1} v_{2}\right) & =0 \\
u\left(v_{1} v_{2}\right) & =\left(u v_{1}\right) v_{2}+\left(u v_{2}\right) v_{1} \\
\left(u_{1} u_{2}\right) v & =u_{1}\left(u_{2} v\right)+u_{2}\left(u_{1} v\right)
\end{aligned}
$$

Fixado $e \in \operatorname{Ip}(A)$, temos que $\operatorname{Ip}(A)=\left\{\epsilon+u+u^{2} \in A ; u \in I_{\epsilon}\right\}$. De fato, é facil ver que $e+u+u^{2} \in \operatorname{Ip}(A)$. para todo $u \in U_{\epsilon}$. Seja, agora $f \in \operatorname{Ip}(A)$. Portanto, $f^{2}=f$. Escrevendo $f=\epsilon+u+v \operatorname{com} u \in U_{\epsilon}$ e $v \in V_{\epsilon}$, temos $\epsilon+u+v=e+u+2 u v+u^{2}+v^{2}$, mas $u+2 u v \in U_{\epsilon}$ e $u^{2}+v^{2} \in V_{e}$. Assim, $u v=0$ e $v=u^{2}+v^{2}$. Multiplicando $v=u^{2}+v^{2}$ por $v$, obtemos $v^{2}=v u^{2}+v^{3}=2 u(u v)=0$. De onde segue que $v=u^{2}$. Portanto, $f=e+u+u^{2}$.

A seguir mostraremos que

$$
x\left(x^{2} y\right)=0
$$

para quaisquer $x$ e $y$ em.$V$. Observemos inicialmente que se $u . t \in U_{e}$ e $v, v \in V_{\epsilon}$ então

$$
u\left(u^{2} t\right)=u\left(u^{2} w\right)=v\left(v^{2} t\right)=v\left(v^{2} w\right)=0
$$

De fato, segue de $(3.40)$ e $(3.47)$ que $u\left(u^{2} t\right)=u^{2}(u t)$. Agora. $u\left(u^{2} t\right)=0$ por (3.27). Por um argumento similar mostram-se as outras igualdades. Portanto, escrevendo $x=u+v \mathrm{e}$ $y=t+w$, temos

$$
\begin{aligned}
x\left(x^{2} y\right) & =2 u((u v) t)+2 u((u v) w)+u\left(v^{2} t\right)+u\left(v^{2} w\right) \\
& +v\left(u^{2} t\right)+v\left(u^{2} w\right)+2 v((u v) t)+2 v((u v) w)
\end{aligned}
$$

Segue de (3.28), (3.43), (3.46) e (3.4T) que

$$
\begin{aligned}
2 u((u v) t) & =2 u((u t) v)-2 u(u(t v)) \\
& =2(u t)(u v)+2 v(u(u t))+u^{2}(t v) \\
& \left.=-v^{\prime}\left(u^{2}\right)\right)
\end{aligned}
$$


Usando (3.2S), (3.42), (3.45) e (3.47), temos

$$
\begin{aligned}
2 u((u v) w) & =2(u(u v)) w-2(u v)(u w) \\
& =\left(u^{2} v\right) u+u^{2}(v w) \\
& =-\left(u^{2}\right) v
\end{aligned}
$$

Segue de (3.28), (3.41) e (3.47) que

$$
\begin{aligned}
2 v((u v) t) & =2(u v)(v t)+2 t((u v) v) \\
& =-v^{2}(u t)+t\left(u v^{2}\right) \\
& =-u\left(v^{2} t\right)
\end{aligned}
$$

De (3.28). (3.41) e (3.46) segue que

$$
\begin{aligned}
2 v((u v) w) & =2(u v)(w v)-2 w((u v) v) \\
& =-v^{2}(u w)-w\left(u v^{2}\right) \\
& =-u\left(v^{2} w\right)
\end{aligned}
$$

Substituindo estas identidades na expressào de $x\left(x^{2} y\right)$, concluímos que $x\left(x^{2} y\right)=0$.

Segue de $(3.27)$ e (3.48) que $\mathrm{N}$ é uma álgebra de Jordan.

Seja $f=e+u_{0}+u_{0}^{2} \in \operatorname{Ip}(A) \operatorname{com} u_{0} \in U_{\epsilon}$. Mostraremos que

$$
\begin{aligned}
U_{f} & =\left\{u+2 u_{0} u ; u \in V_{e}\right\} \\
V_{f} & =\left\{v-2 u_{0} u: v \in V_{\epsilon}\right\}
\end{aligned}
$$

Seja $u^{\prime} \in L_{f}$. Escrevamos $u^{\prime}=u+v$, onde $u \in l_{\epsilon}^{*}$ e $v \in V_{\epsilon}$. Portanto.

$$
0=f u^{\prime}-\frac{1}{2} u^{\prime}=u_{0} v+u_{0}^{2} u+u_{0} u+u_{0}^{2} v-\frac{1}{2} v
$$

Temos que $u_{0} v+u_{0}^{2} u \in U_{\epsilon}$ e $u_{0} u+u_{0}^{2} v-\frac{1}{2} v \in V_{e}$. De onde segue que $u_{0} v=-u_{0}^{2} u$ e $v=2 u_{0} u+2 u_{0}^{2} v$. Assim, usando (3.42) e (3.48). temos

$$
\begin{aligned}
v & =2 u_{0} u+4 u_{0}\left(u_{0} v\right) \\
& =2 u_{0} u-4 u_{0}\left(u_{0}^{2} u\right) \\
& =2 u_{0} u
\end{aligned}
$$


Portanto, $u^{\prime}=u+2 u_{0} u$. Por outro lado, usando (3.2 $)$ ) e (3.43). temos, para todo $u \in U_{\epsilon}$, que $f\left(u+2 u_{0} u\right)=\frac{1}{2}\left(u+2 u_{0} u\right)$. Concluímos que $U_{f}=\left\{u+2 u_{0} u: u \in U_{e}\right\}$. Seja agora $\imath^{\prime} \in V_{f}$ e sejam $u \in U_{\epsilon}$ e $v \in V_{\epsilon}$ tais que $v^{\prime}=u+v$.

$$
0=f v^{\prime}=\frac{1}{2} u+u_{0} v+u_{0}^{2} u+u_{0} u+u_{0}^{2} v
$$

Temos que $\frac{1}{2} u+u_{0} v+u_{0}^{2} u \in U_{\epsilon}$ e $u_{0} u+u_{0}^{2} v \in V_{e}$. Portanto. $v^{\prime}=v-2 u_{0} v$. Agora, segue de $(3.27)$ e $(3.42)$ que $f\left(v-2 u_{0} v\right)=0$. Logo, $V_{f}=\left\{v-2 u_{0} v ; v \in V_{e}\right\}$.

Dados $\epsilon, f \in \operatorname{Ip}(A)$, sejam as funçòes

$$
\begin{aligned}
\sigma(u) & =u+2 u_{0} u \\
\tau(v) & =v-2 u_{0} v
\end{aligned}
$$

onde $f=\epsilon+u_{0}+u_{0}^{2} \operatorname{com} u_{0} \in U_{\epsilon}$. É claro que $\sigma$ e $\tau$ são sobrejetoras. Se $u \in U_{e}$ e $v \in V_{\epsilon}$ sào tais que $\sigma(u)=T(v)=0$, entào $u=-2 u_{0} u \in L_{\epsilon}^{i} \cap V_{\epsilon}$. Logo, $u=0$. Analogamente, $v=0$. Portanto. $\sigma$ e $\tau$ são injetoras. Assim $\sigma$ e $\tau$ sào isomorfismos de espaços vetoriais. Conseqüentemente $U$ e $V$ têm dimensão invariante quanto à escolha do idempotente. $\mathrm{O}$ isomorfismo de espaço vetorial $\varphi: A \rightarrow A$ definido por $\varphi(\alpha e+u+v)=\alpha f+\sigma(u)+\tau(v)$ é a transformação de Peirce de $A$ a associada a $\epsilon$ e a $u_{0}$.

São também isomorfismos de espaços vetoriais os operadores lineares $\xi: U_{e} \rightarrow U_{e}$ e $\zeta: V_{e} \rightarrow V_{e}$ definidos por

$$
\begin{aligned}
& \xi(u)=u-2 u_{0}^{2} u \\
& \zeta(v)=v+2 u_{0}^{2} v
\end{aligned}
$$

De fato, se $\xi(u)=0$, entào $u=2 u_{0}^{2} u$. Multiplicando esta igualdade por $u_{0}$ e, usando (3.48), temos que $u_{0} u=2 u_{0}\left(u_{0}^{2} u\right)=0$. Assim. segue de (3.43) que $u=-4 u_{0}\left(u_{0} u\right)=0$. Portanto, $\xi$ é injetora. Agora se $\zeta(v)=0$, então $v=2 u_{0}^{2} v$. Analogamente. $u_{0} v=0$. Portanto, segue de $(3.42)$ que $v=0$.

Proposição 3.6 As funçòes $\sigma . \tau, \xi \in \zeta$ satisfazem as seguintes condiçòes

(a) $\sigma\left(u_{1}\right) \sigma\left(u_{2}\right)=\tau\left(\xi\left(u_{1}\right) \xi\left(u_{2}\right)\right)$;

(b) $\sigma(u) \tau(v)=\sigma(\xi(u) \dot{\zeta}(v))$ :

(c) $\tau\left(v_{1}\right) \tau\left(v_{2}\right)=\tau\left(\zeta\left(\iota_{1}\right) \zeta\left(v_{2}\right)\right)$.

Demonstraçào: Segue de (3.2S) e (3.44) que $\sigma\left(u_{1}\right) \sigma\left(u_{2}\right)=u_{1} u_{2}-2 u_{0}\left(u_{1} u_{2}\right)-2 u_{0}^{2}\left(u_{1} u_{2}\right)$. Esaudu (3.26). (3.2S) e (3.ti) prora-se que $\xi\left(u_{1}\right) \xi\left(u_{2}\right)=u_{1} u_{2}-2 u_{0}^{2}\left(u_{1} u_{2}\right)$. Agora, segue 
de (3.48) que $\sigma(u) \tau(v)=\tau\left(\xi\left(u_{1}\right) \xi\left(u_{2}\right)\right)$. De (3.28) e (3.47) segue que $\sigma(u) \tau(v)=u v^{\circ}+$ $2 u_{0}(u v)+2 u_{0}^{2}(u v)$. Usando (3.26), (3.2S) e (3.46) obtemos $\xi(u) \zeta^{\circ}(v)=u v+2 u_{0}^{2}(u v) \mathrm{e}$ segue de (3.48) que $\sigma(u) \tau(v)=\sigma(\xi(u) \zeta(v))$. A partir de (3.28) e (3.46) mostra-se que $\tau\left(v_{1}\right) \tau\left(v_{2}\right)=v_{1} v_{2}-2 u_{0}\left(v_{1} v_{2}\right)-2 u_{0}^{2}\left(v_{1} v_{2}\right)$. Usando (3.26), (3.28) e (3.45) provamos que $\zeta\left(v_{1}\right) \zeta\left(v_{2}\right)=v_{1} v_{2}-2 u_{0}^{2}\left(v_{1} v_{2}\right)$. Finalmente, segue de (3.48) que $\tau\left(v_{1}\right) \tau\left(v_{2}\right)=\tau\left(\zeta\left(v_{1}\right) \zeta\left(v_{2}\right)\right)$.

Corolário 3.2 Sejam $X, X_{1}, X_{2} \subseteq U$ e W. $W_{1}, W_{2} \subseteq V$ subespaços de $A=I$ ie $\oplus U \subseteq V$. Então

(a) $\sigma\left(X_{1}\right) \sigma\left(X_{2}\right)=\tau\left(\xi\left(X_{1}^{\prime}\right) \xi\left(X_{2}^{\prime}\right)\right)$;

(b) $\sigma\left(X^{\prime}\right) \tau\left(W^{\prime}\right)=\sigma\left(\xi\left(X^{\prime}\right) \zeta(W)\right)$;

(c) $\tau\left(W_{1}\right) \tau\left(W_{2}\right)=\tau\left(\zeta\left(W_{1}\right) \zeta\left(W_{2}\right)\right)$.

Na Proposiçào seguinte mostramos que os P-subespaços de $A$ absorvem produtos por IV.

Proposição 3.7 Todo P-subespaço $p$ de A satisfa $V p \subseteq p$.

Demonstraçào: Faremos a demonstraçào apenas para um P-monômio $m$. Temos que

$$
\begin{aligned}
V U \subseteq U \\
V^{2} \subseteq V
\end{aligned}
$$

Se $\partial m \geq 2$ então ocorrer uma das três possibilidacles:

$$
\begin{aligned}
m & =\mu \nu \\
m & =\mu_{1} \mu_{2} \\
m & =\nu_{1} \nu_{2}
\end{aligned}
$$

onde $\mu, \mu_{1}, \mu_{2} \subseteq U$ e $\nu, \nu_{1}, \nu_{2} \subseteq V$ sào $\mathrm{P}$-monômios de grau menor ou igual a $\partial \mathrm{m}$. I $\mathrm{Lm}$ gerador de $V(\mu \nu)$ é da forma $v(u w)$, onde $v \in V . u \in \mu$ e $w \in \nu$. Segue de (3.46) que

$$
v(u w)=u(v w)-w(u v) \in \mu(V \nu)+\nu(V \mu)
$$

Temos que $I\left(\mu_{1} \mu_{2}\right)=\left\langle v\left(u_{1} u_{2}\right) ; v \in V, u_{1} \in \mu_{1}, u_{2} \in \mu_{2}\right\rangle$. Usando (3.4T) obtemos

$$
v\left(u_{1} u_{2}\right)=u_{1}\left(u_{2} v\right)+u_{2}\left(u_{1} v\right) \in \mu_{1}\left(\mu_{2} V\right)+\mu_{2}\left(\mu_{1} V\right)
$$

Finalmente. $V\left(\nu_{1} \nu_{2}\right)$ é gerado por elementos da forma $v\left(w_{1} u_{2}\right)$, onde $v \in V, u_{1} \in \nu_{1}$ e $u_{2} \in \nu_{2}$. Por $(3.45)$ tem-se

$$
v\left(w_{1} w_{2}\right)=-u_{1}\left(w_{2} v\right)-w_{2}\left(v^{\prime} u_{1}\right) \in \nu_{1}\left(\nu_{2} V\right)+\nu_{2}\left(V \nu_{1}\right)
$$

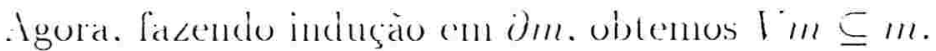


Corolário 3.3 Para quaisquer P-subespaços $g \subseteq U \in h \subseteq V$ tem-se

$$
\begin{aligned}
\xi(g) & =g \\
\zeta(h) & =h
\end{aligned}
$$

Demonstração: Seja $u \in g$. Segue da proposição anterior que $\xi(u)=u-2 u_{0}^{2} u \in g+V g=g$. Logo, $\xi(g) \subseteq g$ e, como $\xi$ é um isomorfismo de espaços vetoriais, $\xi(g)=g$. Analogamente, mostra-se que $\zeta(h)=h$.

Agora pode-se demonstrar de maneira análoga às seções anteriores o seguinte teorema, onde $\varphi$ é uma transformaçào de Peirce de $A$.

Teorema 3.5 Seja A uma álgebra bárica satisfazendo (3.25). Entào

(1) Todo P-subespaço $p$ de A satisfaz $\varphi\left(p_{e}\right)=p_{f}$. Em particular. todo P-subespaço tem dimensào invariante.

(2) Para todo P-subespaço $p$ de A as afirmaçòes seguintes sào equicalentes:

(2.1) p é invariante:

(2.2) $U p \subseteq p$;

(2.3) p é ideal.

Demonstração: Basta demonstrar a parte (1) para um P-monômio $m$. Sejam $\epsilon$ e $f=$ $e+u_{0}+u_{0}^{2}$ idempotentes de $A$ com $u_{0} \in U_{e}$. Como $\sigma$ e $\tau$ sào isomorfismos, temos

$$
\begin{gathered}
U_{f}=\sigma\left(U_{\epsilon}\right)=\varphi\left(U_{e}\right) \\
V_{f}=\tau\left(V_{\epsilon}\right)=\varphi\left(V_{\epsilon}\right)
\end{gathered}
$$

Suponhamos que o resultado é válido para todo P-monômio de grau menor ou igual a $k$ e seja $m$ um P-monômio com $\partial m=k+1$. Existem três possibilidades: $m=\mu \nu, m=\mu_{1} \mu_{2}$, $m=\nu_{1} \nu_{2}$, onde $\mu, \mu_{1} . \mu_{2} \in U^{*}$ e $\nu, \nu_{1}, \nu_{2} \in V$ são P-monômios de grau menor ou igual a $k$. Se $m=\mu \nu$ entào.

$$
m_{f}=\mu_{f} \nu_{f}=\sigma\left(\mu_{e}\right) \tau\left(\nu_{e}\right)
$$

Assim, pelo Corolário 3.2 temos

$$
m_{f}=\sigma\left(\xi\left(\mu_{e}\right) \zeta\left(\nu_{e}\right)\right)=\sigma\left(\mu_{e} \nu_{e}\right)=\sigma\left(m_{e}\right)=\varphi\left(m_{e}\right)
$$

Os outros casos são análogos.

A demonstraçào de que (2.2) é equivalente a $(2.2)$ é completamente análoga a prova de Teorema 2.2. Prova-se que $(2.2)$ é equivalente a $(2.3)$ da mesma maneira como foi demonstradio que (c) é equiralente a (b) na Proposiçào 2.i. 


\section{4 Álgebras satisfazendo $\left(x^{2}\right)^{2}=\omega\left(x^{3}\right) x$}

Seja $(A, \omega)$ uma álgebra bárica, onde $A$ é comutativa, não associativa e de dimensão finita sobre o corpo $K^{\prime} \operatorname{com} \operatorname{car}\left(K^{\prime}\right) \neq 2$ e 3 . Etherington mostrou em [4] que se $\left(A, \omega^{\prime}\right)$ satisfaz

$$
x^{3}-(1+\gamma) \omega(x) x^{2}+\gamma \omega\left(x^{2}\right) x=0
$$

com $\gamma \in K$, entào (A.w) também satisfaz

$$
\left(x^{2}\right)^{2}-(1+2 \gamma) \omega\left(x^{2}\right) x^{2}+2 \gamma \omega\left(x^{3}\right) x=0
$$

que é a correspondente train equação para as três primeiras potências plenárias. Em [17] Walcher demonstrou que $(3.51)$ e (3.52) sào equivalentes exceto para $\gamma=0$ e $\gamma=-\frac{1}{2}$. Se

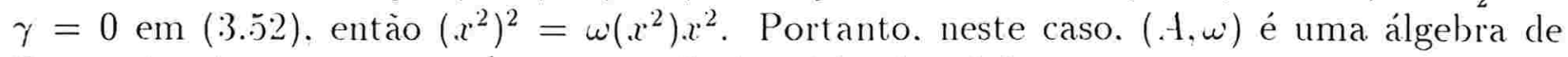
Bernstein. Agora. se ${ }_{i}=-\frac{1}{2}$, entào a álgebra ( $A$.w) satisfaz

$$
\left(x^{2}\right)^{2}=\omega\left(x^{3}\right) x
$$

Estas álgebras também são estudadas em [1], [10], [11] e [21].

Toda álgebra A que satisfaz (3.53) possui um idempotente dado por $\epsilon=\left(d^{3}\right)^{3}$ onde $\omega(d)=1$ (ver [21]). Cada idempotente $\epsilon$ de $A$ determina uma decomposição de Peirce $A=K e \oplus U_{\epsilon} \boxminus V_{\epsilon}$, onde

$$
\begin{aligned}
& U_{e}=\left\{u \in \operatorname{ker} \omega: \epsilon u=\frac{1}{2} u\right\} \\
& V_{e}=\left\{v \in \operatorname{ker} \omega ; \epsilon v=-\frac{1}{2} v\right\}
\end{aligned}
$$

Estes subespaços satisfazem

$$
U V \subseteq U, \quad U^{2} \subseteq V \quad V^{2} \subseteq V
$$

C'omo nas seçòes anteriores, usamos a notaçào $V=\operatorname{ker} \omega$ e temos $N=U \oplus V$.

Segue de (3.53) que para quaisquer $x \in N$

$$
\left(x^{2}\right)^{2}=0
$$

Assim. A também satisfaz as identidades (2). (3) e (4). Outra identidacle válida nas álgebras que satisfazem (3.5) é dada por

$$
x_{1}\left(x_{1}^{2} \cdot x_{2}\right)=0
$$


Segue de (2) e (3.56) que .1 é uma álgebra de Jordan.

Para quaisquer $u \in L^{*}$ e $v \in V$ temos

$$
\begin{aligned}
u^{3} & =0 \\
v^{3} & =0 \\
u^{2} v & =2 u(u v) \\
u v^{2} & =2 v(v u)
\end{aligned}
$$

Por linearização destas identidades, obtemos

$$
\begin{aligned}
u_{1}\left(u_{2} u_{3}\right)+u_{2}\left(u_{3} u_{1}\right)+u_{3}\left(u_{1} u_{2}\right) & =0 \\
v_{1}\left(v_{2} v_{3}\right)+v_{2}\left(v_{3} v_{1}\right)+v_{3}\left(v_{1} v_{2}\right) & =0 \\
\left(u_{1} u_{2}\right) v & =u_{1}\left(u_{2} v\right)+u_{2}\left(u_{1} v\right) \\
u\left(v_{1} v_{2}\right) & =\left(u v_{1}\right) v_{2}+\left(u v_{2}\right) v_{1}
\end{aligned}
$$

O conjunto dos idempotentes de peso 1 de A é dado por

$$
\operatorname{Ip}(A)=\left\{\epsilon+u+\frac{1}{2} u^{2} ; u \in U_{\epsilon}\right\}
$$

e, se $f=\epsilon+u_{0}+\frac{1}{2} u_{0}^{2} \cdot u_{0} \in U_{e}$, é outro idempotente, entào

$$
\begin{aligned}
& U_{f}=\left\{u+u_{0} u ; u \in U_{\epsilon}\right\} \\
& V_{f}=\left\{v-u_{0} v ; v \in V_{\epsilon}\right\}
\end{aligned}
$$

A demonstraçào da próxima proposiçào é análoga a da Proposiçào 3.7.

Proposição 3.8 Ś $p$ é um P-subespaço de uma álgebra que satisfaz (3.5.3). então $V p \subseteq p$.

Para quaisquer $u_{0} \in L^{*}$ e $\alpha, \beta \in K$ consideraremos o operador linear $T_{(\alpha, \beta)}: N \rightarrow \Lambda$ definido por

$$
T_{(\alpha, \beta)}(x)=x+\alpha u_{0} x+\beta u_{0}^{2} x
$$

Tais operadores satisfazem as seguintes propriedades:

Lema 3.7 Para quaisquer $u_{0} \in U \in \alpha . \beta \in K^{\circ}$. lem-se

(a) $T_{(a, 3)}$ é um automorfismo de espaço vetorial:

(b) $T_{(0,3)}(p)=p$ para todo P-subespaço $p d e$. 
Demonstraçaio: Seja $x=u+v \in N$ com $u \in U^{r}$ e $v \in V$ tal que $T_{(\alpha, \beta)}(x)=0$. Assim $u+v+\alpha u_{0}(u+v)+\beta u_{0}^{2}(u+v)=0$, de onde segue que

$$
\begin{aligned}
& u+\alpha u_{0} v+3 u_{0}^{2} u=0 \\
& v+\alpha u_{0} u+3 u_{0}^{2} v=0
\end{aligned}
$$

Multiplicando estas identidades por $u_{0}$ e usando (3.56), (3.59) e (3.61), obtemos

$$
\begin{aligned}
& u_{0} u+\frac{1}{2} \alpha u_{0}^{2} v=0 \\
& u_{0} v-\frac{1}{2} a u_{0}^{2} u=0
\end{aligned}
$$

Novamente. multiplicando por $u_{0}$ estas duas últimas igualdades e usando (3.56). (3.5.9) e (3.61). temos $u_{0}^{2} u=u_{0}^{2} v=0$. Logo $u_{0} u=u_{0} v=0$. Portanto. $u=v=0$. Assim $T_{(a, 3)}$ é injetor e portanto, é um isomorfismo. Seja $x \in p$. Como $u_{0}^{2} \in V$. segue da Proposiçào 3.8 que

$$
T_{(0, \beta)}(x)=x+3 u_{0}^{2} x \in p+V p \subseteq p
$$

Portanto. $T_{(0, \beta)}(p) \subseteq p$ e, como $T_{(\alpha, \beta)}$ é injetor. temos a igualdade.

Dados $\epsilon, f \in \operatorname{Ip}(A), \operatorname{com} f=e+u_{0}+\frac{1}{2} u_{0}^{2}, u_{0} \in U_{e}$, usaremos as notações: $\sigma=\left.T_{(1,0)}\right|_{U_{e}}$, $\tau=\left.T_{(-1,0)}\right|_{V_{e}}, \xi=\left.T_{\left(0,-\frac{1}{2}\right)}\right|_{U_{e}}, \zeta=\left.T_{\left(0, \frac{1}{2}\right)}\right|_{V_{e}}$. Temos que $\sigma: U_{e} \rightarrow U_{f}, \tau: V_{e} \rightarrow V_{f}, \xi: U_{\epsilon} \rightarrow U_{\epsilon}$ e $\zeta: V_{\epsilon} \rightarrow V_{f}$. O isomorfismo de espaço vetorial $\uparrow: A \rightarrow A$ definido por $\varphi(\alpha e+u+v)=$ $\alpha f+\sigma(u)+\tau(v)$ é a transformação de Peirce de A a associada a $\epsilon$ e a $u_{0}$.

Proposição 3.9 As funçòes $\sigma, \tau, \xi$ e $\zeta$ satisfazem as identidades

(a) $\sigma\left(u_{1}\right) \sigma\left(u_{2}\right)=\tau\left(\xi\left(u_{1}\right) \xi\left(u_{2}\right)\right)$;

(b) $\sigma(u) \tau(v)=\sigma(\xi(u) \zeta(v))$;

(c) $\tau\left(v_{1}\right) \tau\left(v_{2}\right)=\tau\left(\zeta\left(v_{1}\right) \zeta\left(v_{2}\right)\right)$.

para quaisquer $u, u_{1}, u_{2} \in U_{\epsilon} \in v, v_{1}, v_{2} \in V_{\epsilon}$.

Finalmente, como nas seçòes anteriores. temos:

Teorema 3.6 Seja A uma álgebra báricas satisfazendo (3.5:3). Entioo 
(1) Todo P-subespaço $p$ de t satisfaz $\varphi\left(p_{e}\right)=p_{f}$. Em particular. todo P-subespaço tem. dimensio invariante.

(2) Para todo P-subespaço p de A as afirmações seguintes sào equivalentes:

(2.1) p é invariante:

(2.2) $U p \subseteq p$;

(2.3) pé ideal.

\subsection{Comentários finais}

Estudamos o problema da invariância de P-subespaços em algébras báricas $(A, \omega)$ satisfazendo as equaçòes

$$
\begin{aligned}
\left(x^{2}\right)^{2} & =\omega\left(x^{3}\right) x \\
\left(x^{2}\right)^{2} & =\omega\left(x^{2}\right) x^{2} \\
\left(x^{2}\right)^{2} & =\omega(x) x^{3} \\
x^{3} & =(1+\gamma) \omega(x) x^{2}-\gamma \omega\left(x^{2}\right) x
\end{aligned}
$$

Observemos que estas classes de álgebras contém as álgebras que satisfazem

$$
\begin{array}{rlr}
x^{2} & =\omega(x) x & \\
x^{3} & =\omega\left(x^{k}\right) x^{r} & k+r=3 \\
\left(x^{2}\right)^{2} & =\omega\left(x^{k}\right) x^{r} & k+r=4
\end{array}
$$

onde $k$ e $r$ sào inteiros positiros. De fato, as álgebras que satisfazem (3.66) são álgebras de Bernstein cujo núcleo é uma zero álgebra (logo seus P-subespaços sào apenas $U, V \mathrm{e}$ $U \oplus V$ e são invariantes). as álgebras que satisfazem (3.6T) são train álgebras de posto 3 ( $\operatorname{com} \gamma=-1$ ou $\hat{\imath}=0)$.

Todas as álgebras báricas consideradas possuem uma decomposição de Peirce da forma $A=K e \oplus U V$ associada a um idempotente. Os subespaços $U$ e $V$ satisfazem relaçòes do tipo

$$
\zeta \subseteq U, \quad U^{2} \subseteq V, \quad V^{2} \subseteq \Omega
$$

onde. dependendo do caso $\Omega=0, \Omega=I . \Omega=V$. O conjunto $\operatorname{Ip}(. A)$ dos elementos idempotentes de peso l é parametrizado através do subespaço I' da seguinte maneira:

$$
\operatorname{Ip}(. A)=\left\{\epsilon+u+\theta u^{2}: u \in I^{\prime}\right\}
$$


com $\theta \in K^{\prime}$. Para cada $u_{0} \in U_{e}$, considerando $f=e+u_{0}+\theta u_{0}^{2}$, temos os isomorfismos de espaços vetoriais $\sigma: U_{e} \rightarrow U_{f}$ e $\tau: V_{e} \rightarrow V_{f}$ definidos por

$$
\begin{aligned}
\sigma(u) & =u+\alpha u_{0} u \\
\tau(v) & =v+\beta u_{0}+\rho u_{0}^{2} v
\end{aligned}
$$

onde $\alpha, 3, \rho \in K$. Temos que $\rho=-1$ para álgebras de Bernstein e, nos outros casos, $\rho=0$. Salvo as álgebras de Bernstein que não vericam (2.18), as outras álgebras consideradas possuem a propriedade de que o seus P-subespaços têm dimensões invariantes. Isso se deve ao fato de que nestas álgebras vale uma tabela da forma

$$
\begin{aligned}
\varphi\left(u_{1}\right) \varphi\left(u_{2}\right) & =\varphi\left(\xi\left(u_{1}\right) \xi\left(u_{2}\right)\right) \\
\varphi(u) \varphi(v) & =\varphi(\xi(u) \zeta(v)) \\
\varphi\left(v_{1}\right) \varphi\left(v_{2}\right) & =\varphi\left(\zeta\left(v_{1}\right) \zeta\left(v_{2}\right)\right.
\end{aligned}
$$

onde $\varphi$ é a transformaçào de Peirce de $A$ associada a $e$ e a $u_{0}$ e $\xi: U_{e} \rightarrow U_{\epsilon}, \zeta: V_{e} \rightarrow V_{\epsilon}$ são isomorfismos de espaços vetoriais definidos por

$$
\begin{aligned}
& \xi(u)=u+\epsilon u_{0}^{2} u \\
& \zeta(v)=v+\delta u_{0}^{2} v
\end{aligned}
$$

onde $\epsilon, \delta \in K^{\prime}$ (nas train álgebras de posto 3 e nas álgebras de Bernstein que satisfazem (2.1S) temos $\delta=0$. isto é, $\zeta$ é a função identidade). A principal propriedade das funções $\xi$ e $\zeta$ é que elas preservam P-subespaços. Dessa maneira, a tabela anterior implica que $\varphi\left(p_{\varepsilon}\right)=p_{f}$. Esta última igualdade nos permitiu também estabelecer uma condiçào necessária e suficiente bem simples para que um P-subespaço seja invariante. 


\section{Referências Bibliográficas}

[1] M. T. Alcalde. C. Burgueño and C. Mallol. Les Pol(n.m)-algèbres: identités polynômiales symétriques des algèbres. Linear Algebra and its Applications, 191:213-234. 199:3.

[2] C. Burgueño and C. Mallol. Morphismes de Peirce et orthogonalité dans les algèbres de Bernstein. Linear. Algebra and its Applications, 219:179-186. 1997.

[3] T. Cortés and F. MIontaner. On the structure of Bernstein algebras. J. London Math. Soc., 51(2):41-52, 1995.

[4] I. M. H. Etherington. Commutative train algebras of ranks 2 and 3. J. London Math. Soc. 15, 136:149. 1940.

[5] S. Gonzalez and C. Martinez. Idempotent elements in a Bernstein algebra. J. London Math. Soc. $42(2): 430-436,1990$.

[6] I. R. Hentzel and L. A. Peresi. Semiprime Bernstein algebras. Arch. Math. 52:5:39543. 1989.

[7] A. Labra, C. Mallol, A. Micali and R. Varro. Sur les algèbres de Bernstein V: les algèbres de Bernstein Jordan faibles. Proceedings of the Edinburgh Mathematical Society. 3:5:35.9-373. 199:2.

[8] M. L. Lelis. Formas P-invariantes em álgebras de Bernstein. PhD thesis, Instituto de Matemática e Estatística da Universidade de Sào Paulo. 1996.

[9] Y. Luubich. Mathematical S'tructures in Population Genetics., Biomathematics. Springer 22,1992 .

[10] C. Malbol and R. Varro. A propos des algèbres iérifiant $x^{[3]}=w(x)^{3} x$. Linear Algebra and its Applications, 225:187-194, 1995. 
[11] C. Mallol and R. Varro. Derivation dans les algèbres de Walcher. Nova Journal of Mathematics. Game Theory, and Algebra. 4(2):129-146, 1996.

[12] C. Mallol and A. Walkhoff. Sur le algèbres vérifiant $x^{3}=\lambda(x) x^{2}$. Nova Journal of Mathematics, Game Theory, and Algebra, 4(2):169-184, 1996.

[13] A. Micali and M. Ouattara. Structure des algèbres de Bernstein. Linear Algebra and its Applications, 218:Ti-88, 1995.

[14] R. B. Gallardo. Sobre as T-álgebras de posto 3 e questóes correlatas. PhD thesis. Instituto de Matemática e Estatística da Universidade de São Paulo, 1995.

[15] R. A. Oliveira. Sobre os P-subespaços em uma train álgebra de posto 3. PhD thesis, Instituto de Matemática e Estatística da Universidade de São Paulo, 1997.

[16] R. D. Schafer. An Introduction to Vonassociative Algebras, Dover Publications. Inc., New York. 1966.

[17] S. WaLCHER. Algebras which satisfy a train equation for the first three plenary powers. Arch. Math., 56:547-5.51, 1991.

[18] S. Walcher. Bernstein algebras which are Jordan algebras. Arch. Math., 50:218-222, 1988.

[19] A. Wörz-Busekros. Algebras in Cienetics, volume 36, Lecture Notes in Biomathematics. Springer-Verlag, Berlin/New York, 1980.

[20] A. Wörz-Busekros. Bernstein algebras. Arch. Math., 48:388-398, 1987.

[21] R. Varro. Algèbres de Bernstein périodiques. Thèse de Doctorat, Université de Montpellier II. 1992. 


\section{Índice de Símbolos}

$(A, \omega)$ - álgebra bárica, 6

$A=L e \oplus U_{\epsilon} \oplus V_{e}$ - decomposiçào de Peirce de A. 6

$A^{*}$ - espaço das formas lineares de $A$ em I, 10

$K^{\prime}-$ corpo. 5

$K[U, V]$ - álgebra de polinômios em $U$ e V. 13

$L-U \cap \operatorname{ann} U, 10$

$P$ - ideal de Etherington, 10

$S_{k, r}-S_{k, U} \oplus S_{r, V}, 22$

$U_{\epsilon}, V_{e}$ - subespaços de uma decomposição de Peirce de $A, T$

$X^{n}$ - potências principais do subespaço $X$, j

$\widetilde{U}-U+U^{2}=\sum_{f \in \operatorname{Ip}(A)} U_{f}, 10$

$\tilde{V}-V+C V+U^{2} V=\sum_{f \in \operatorname{Ip}(A)} V_{f}, 10$

$\mathcal{P}$ - subconjunto de $K\left[\mathrm{C}^{*} . \mathrm{V}\right] .13$

$\omega$ - funçào peso. 6

$\bar{A}$ - álgebra quociente, 6

$\tilde{p}-p(\tilde{U}, \tilde{V}), 20$

$\partial p$ - grau de $p .14$

$a^{n}$ - potências principais de $a .5$

$e$-idempotente de A, 6

$o(p)$ - ordem de $p, 14$ 


\section{Índice Remissivo}

\author{
Álgebra bárica. 6 \\ de Bernstein. 6 \\ excepcional. 8 \\ normal. 8 \\ nuclear. 8 \\ de Bernstein-Jordan, 11 \\ de Bernstein-Jordan fracas, 58 \\ de Jordan. 11 \\ de polinômios, 13 \\ satisfazendo $\left(x^{2}\right)^{2}=\omega(x) x^{3}, 62$ \\ satisfazendo $\left(x^{2}\right)^{2}=\omega\left(x^{3}\right) x, 69$ \\ satisfazendo $x^{3}=\lambda(x) x^{2}, 53$ \\ satisfazendo $x^{3}=\lambda(x) x^{2} \operatorname{com} \lambda\left(A^{2}\right)=$ \\ 0,54 \\ train álgebra, 51 \\ train álgebra de posto 3,51
}

Decomposição de Peirce, 6, 51, 54, 58, 63,

69

Função peso. 6

Grau, 14

Ideal de Etherington. 10

Núcleo, 6

Ordem. 14

P-monômios. 1:3

P-subespaços. 13 invariantes, 14

que têm dimensão invariante. 14

Peso, 6

Posto, 51

Potências principais, 5

Subespaços monomiais, 13

polinomiais, 13

Tipo, 8

Train polinômio, 51

Transformação de Peirce, 32, 56, 59, 66. 68 